\title{
Supporting Information: Size Dependent Fragmentation Chemistry of Short Doubly Protonated Tryptic Peptides
}

\author{
Shanshan Guan ${ }^{\dagger, \|,}$ Benjamin J. Bythell ${ }^{\dagger, \|^{*}}$ \\ ${ }^{\dagger}$ Department of Chemistry and Biochemistry, Ohio University, 307 Chemistry Building, Athens, OH 45701 \\ "Department of Chemistry and Biochemistry, University of Missouri-St. Louis, 1 University Blvd, St. Louis, MO \\ 63121
}

\section{AUTHOR INFORMATION}

Corresponding Author

*Benjamin J. Bythell, bythell@ohio.edu 


\section{Table of content}

Table S1: Relative energies of the transition structures of $a_{1}-y_{N-1}$ and $b_{2}-y_{N-2}$ pathways for each doubly protonated peptides calculated at the B3LYP/6-31+G(d,p) level of theory.

Table S2. Relative energies of the transition structures of $a_{1}-y_{N-1}$ and $b_{2}-y_{N-2}$ pathways for each doubly protonated peptides calculated at the M06-2X/6-31+G(d,p) level of theory.

Table S3. Relative energies of the consecutive $b_{2}-a_{2}$ reaction (Scheme S1) transition structures for each doubly protonated peptides calculated at the B3LYP/6-31+G(d,p) level of theory. The doubly protonated precursor peptide ion GM is the reference value. Note that the $b_{2}-a_{2}$ barriers for the $\left[\mathrm{A}_{2} \mathrm{R}+2 \mathrm{H}\right]^{2+}$ and $\left[\mathrm{A}_{3} \mathrm{R}+2 \mathrm{H}\right]^{2+}$ species requires less energy than the preceding $\mathrm{b}_{2}-\mathrm{y}_{\mathrm{N}-2}$ transition structures $\left(\Delta \mathrm{E}_{\mathrm{el}+\mathrm{ZEP}, 0 \mathrm{~K}}=144.1\right.$ and $151.9 \mathrm{~kJ} \mathrm{~mol}^{-1}$ respectively, Table $\left.\mathrm{S} 1\right)$.

Table S4. Relative energies of the consecutive $b_{2}-a_{2}$ reaction (Scheme S1) transition structures for each doubly protonated peptide calculated at the M06-2X/6-31+G(d,p) level of theory. . The doubly protonated precursor peptide ion $\mathrm{GM}$ is the reference value. Note that the $\mathrm{b}_{2}-\mathrm{a}_{2}$ barriers for the $\left[\mathrm{A}_{2} \mathrm{R}+2 \mathrm{H}\right]^{2+}$ and $\left[\mathrm{A}_{3} \mathrm{R}+2 \mathrm{H}\right]^{2+}$ species requires less energy than the preceding $\mathrm{b}_{2}-\mathrm{y}_{\mathrm{N}-2}$ transition structures $\left(\triangle \mathrm{E}_{\mathrm{el}+\mathrm{ZEP}, 0 \mathrm{~K}}=151.0\right.$ and $155.5 \mathrm{~kJ} \mathrm{~mol}^{-1}$ respectively, Table $\left.\mathrm{S} 2\right)$.

Table S5. Relative energies of the consecutive $b_{2}-a_{2}$ reaction (Scheme S1) transition structures for each doubly protonated peptides calculated at the B3LYP/6-31+G(d,p) level of theory. The doubly protonated precursor peptide ion GM is the reference value. Note that the $b_{2}-a_{2}$ barriers for the $\left[\mathrm{G}_{2} \mathrm{R}+2 \mathrm{H}\right]^{2+}$, and $\left[\mathrm{G}_{3} \mathrm{R}+2 \mathrm{H}\right]^{2+}$ species requires less energy than the preceding $\mathrm{b}_{2}-\mathrm{y}_{\mathrm{N}-2}$ transition structures $\left(\triangle \mathrm{E}_{\mathrm{el}+\mathrm{ZEP}, 0 \mathrm{~K}}=151.8\right.$ and $159.7 \mathrm{~kJ} \mathrm{~mol}^{-1}$ respectively, Table $\left.\mathrm{S} 1\right)$.

Table S6. Relative energies of the consecutive $\mathrm{b}_{2}$ - $\mathrm{a}_{2}$ reaction (Scheme S1) transition structures for each doubly protonated peptide calculated at the M06-2X/6-31+G(d,p) level of theory. . The doubly protonated precursor peptide ion GM is the reference value. Note that the $b_{2}-a_{2}$ barriers for the $\left[\mathrm{G}_{2} \mathrm{R}+2 \mathrm{H}\right]^{2+}$, and $\left[\mathrm{G}_{3} \mathrm{R}+2 \mathrm{H}\right]^{2+}$ species requires less energy than the preceding $\mathrm{b}_{2}-\mathrm{y}_{\mathrm{N}-2}$ transition structures $\left(\triangle \mathrm{E}_{\mathrm{el}+\mathrm{ZEP}, 0 \mathrm{~K}}=151.4\right.$ and $154.9 \mathrm{~kJ} \mathrm{~mol}^{-1}$ respectively, Table $\left.\mathrm{S} 2\right)$.

Figure S1. Left column: Relative abundances of $\mathrm{y}_{\mathrm{N}-1}$ and $\mathrm{y}_{\mathrm{N}-2}$ peaks as a function of collision energy for $\left[\mathrm{G}_{\mathrm{x}} \mathrm{R}+2 \mathrm{H}\right]^{2+}$. Right column: RRKM plots for $\mathrm{b}_{2}-\mathrm{y}_{\mathrm{N}-2}$ and $\mathrm{a}_{1}-\mathrm{y}_{\mathrm{N}-1}$ reactions for $\left[\mathrm{G}_{\mathrm{x}} \mathrm{R}+2 \mathrm{H}\right]^{2+}$ using data from M06-2X/6-31+G(d,p) calculations of global minima and TSs.

Figure S2. Left column: Relative abundances of $\mathrm{y}_{\mathrm{N}-1}$ and $\mathrm{y}_{\mathrm{N}-2}$ peaks as a function of collision energy for $\left[\mathrm{A}_{\mathrm{x}} \mathrm{R}+2 \mathrm{H}\right]^{2+}$. Right column: RRKM plots for $\mathrm{b}_{2}-\mathrm{y}_{\mathrm{N}-2}$ and $\mathrm{a}_{1}-\mathrm{y}_{\mathrm{N}-1}$ reactions for $\left[\mathrm{A}_{\mathrm{x}} \mathrm{R}+2 \mathrm{H}\right]^{2+}$ using data from B3LYP/6-31+G(d,p) calculations of global minima and TSs.

Figure S3. Left column: Relative abundances of $\mathrm{y}_{\mathrm{N}-1}$ and $\mathrm{y}_{\mathrm{N}-2}$ peaks as a function of collision energy for $\left[\mathrm{G}_{\mathrm{x}} \mathrm{R}+2 \mathrm{H}\right]^{2+}$. Right column: RRKM plots for $\mathrm{b}_{2}-\mathrm{y}_{\mathrm{N}-2}$ and $\mathrm{a}_{1}-\mathrm{y}_{\mathrm{N}-1}$ reactions for $\left[\mathrm{G}_{\mathrm{x}} \mathrm{R}+2 \mathrm{H}\right]^{2+}$ using data from M06-2X/6-31+G(d,p) calculations of global minima and TSs. 
Figure S4. Left column: Relative abundances of $\mathrm{y}_{\mathrm{N}-1}$ and $\mathrm{y}_{\mathrm{N}-2}$ peaks as a function of collision energy for $\left[\mathrm{A}_{\mathrm{x}} \mathrm{R}+2 \mathrm{H}\right]^{2+}$. Right column: RRKM plots for $\mathrm{b}_{2}-\mathrm{y}_{\mathrm{N}-2}$ and $\mathrm{a}_{1}-\mathrm{y}_{\mathrm{N}-1}$ reactions for $\left[\mathrm{A}_{\mathrm{x}} \mathrm{R}+2 \mathrm{H}\right]^{2+}$ using data from B3LYP/6-31+G(d,p) calculations of global minima and TSs.

Figure S5. Left column: Relative abundances of $\mathrm{y}_{\mathrm{N}-1}$ and $\mathrm{y}_{\mathrm{N}-2}$ peaks as a function of collision energy for $\left[\mathrm{G}_{\mathrm{x}} \mathrm{R}+2 \mathrm{H}\right]^{2+}$. Right column: RRKM plots for $\mathrm{b}_{2}-\mathrm{y}_{\mathrm{N}-2}$ and $\mathrm{a}_{1}-\mathrm{y}_{\mathrm{N}-1}$ reactions for $\left[\mathrm{G}_{\mathrm{x}} \mathrm{R}+2 \mathrm{H}\right]^{2+}$ using data from B3LYP/6-31+G(d,p) calculations of global minima and TSs.

Figure S6. Global minima of doubly protonated peptides calculated at the B3LYP/6-31+G(d,p) level of theory.

Figure S7. Global minima of doubly protonated peptides calculated at the M062X/6-31+G(d,p) level of theory. Note the greater degree of folding relative to the B3LYP congeners. i.e., improved charge-solvation at the cost of decreasing the distances between the charges.

Figure S8. Transition Structures of $\mathrm{a}_{1}-\mathrm{y}_{\mathrm{N}-1}$ and $\mathrm{b}_{2}-\mathrm{y}_{\mathrm{N}-2}$ reactions for $\left[\mathrm{G}_{\mathrm{x}} \mathrm{R}+\mathrm{H}\right]^{+}$calculated at the M06-2X/6-31+G(d,p) level of theory.

Figure S9. Transition Structures of $a_{1}-y_{N-1}$ and $b_{2}-y_{N-2}$ reactions for $\left[A_{x} R+H\right]^{+}$calculated at the B3LYP/6-31+G(d,p) level of theory.

Figure S10. Transition Structures of $\mathrm{a}_{1}-\mathrm{y}_{\mathrm{N}-1}$ and $\mathrm{b}_{2}-\mathrm{y}_{\mathrm{N}-2}$ reactions for $\left[\mathrm{G}_{\mathrm{x}} \mathrm{R}+\mathrm{H}\right]^{+}$calculated at the B3LYP/6-31+G(d,p) level of theory.

Scheme S1. Generic $b_{2}-a_{2}$ reaction. Note that the post-decarbonylation, $a_{2}$ ion cyclization reaction is sequence dependent.

Images and coordinates of global minima and transition structures discussed in this manuscript. 
Table S1. Relative energies of the transition structures of $\mathrm{a}_{1}-\mathrm{y}_{\mathrm{N}-1}$ and $\mathrm{b}_{2}-\mathrm{y}_{\mathrm{N}-2}$ pathways for each doubly protonated peptides calculated at the B3LYP/6-31+G(d,p) level of theory.

\begin{tabular}{|c|c|c|c|c|c|c|}
\hline $\begin{array}{c}\text { Doubly } \\
\text { Protonated } \\
\text { Peptide }\end{array}$ & Reaction & $E_{\mathrm{el}} / \mathbf{H}$ & $\begin{array}{c}\Delta E_{\text {el+ZPE, } 0 \mathrm{~K}} \\
\mathrm{~kJ} \mathrm{~mol}^{-1}\end{array}$ & $\begin{array}{c}\Delta H_{298 \mathrm{~K}} \\
\mathrm{~kJ} \mathrm{~mol}^{-1}\end{array}$ & $\begin{array}{c}\Delta G_{298 \mathrm{~K}} \\
\mathrm{~kJ} \mathrm{~mol}^{-1}\end{array}$ & $\begin{array}{c}\Delta S_{298 K} \\
\mathrm{~J} \mathrm{~K}^{-1} \mathrm{~mol}^{-1}\end{array}$ \\
\hline \multirow{2}{*}{ AAR } & $\mathrm{a}_{1}-\mathrm{y}_{2}$ & -1101.943153 & 178.3 & 181.0 & 171.0 & 33.3 \\
\hline & $\mathrm{b}_{2}-\mathrm{y}_{1}$ & -1101.959436 & 144.1 & 142.7 & 148.3 & -18.9 \\
\hline \multirow{2}{*}{$\mathrm{A}_{3} \mathrm{R}$} & $\mathrm{a}_{1}-\mathrm{y}_{3}$ & -1349.311815 & 159.4 & 164.8 & 148.7 & 53.9 \\
\hline & $\mathrm{b}_{2}-\mathrm{y}_{2}$ & -1349.318077 & 151.9 & 152.5 & 151.6 & 2.8 \\
\hline \multirow{2}{*}{$\mathrm{A}_{4} \mathrm{R}$} & $a_{1}-y_{4}$ & -1596.672214 & 166.5 & 170.9 & 158.5 & 41.5 \\
\hline & $\mathrm{b}_{2}-\mathrm{y}_{3}$ & -1596.692014 & 122.7 & 122.9 & 119.5 & 11.3 \\
\hline \multirow{2}{*}{$\mathrm{A}_{5} \mathrm{R}$} & $a_{1}-y_{5}$ & -1844.03016 & 165.1 & 169.7 & 154.9 & 49.7 \\
\hline & $\mathrm{b}_{2}-\mathrm{y}_{4}$ & -1844.049931 & 121.7 & 121.8 & 121.9 & -0.2 \\
\hline \multirow{2}{*}{ GGR } & $a_{1}-y_{2}$ & -1023.294996 & 190.4 & 194.6 & 181.0 & 45.6 \\
\hline & $\mathrm{b}_{2}-\mathrm{y}_{1}$ & -1023.312541 & 151.8 & 151.7 & 151.5 & 0.5 \\
\hline \multirow{2}{*}{$\mathrm{G}_{3} \mathrm{R}$} & $a_{1}-y_{3}$ & -1231.341039 & 181.5 & 185.9 & 174.2 & 39.5 \\
\hline & $b_{2}-y_{2}$ & -1231.352572 & 159.7 & 159.4 & 161.7 & -7.5 \\
\hline \multirow{2}{*}{$\mathrm{G}_{4} \mathrm{R}$} & $\mathrm{a}_{1}-\mathrm{y}_{4}$ & -1439.385426 & 171.0 & 176.2 & 160.2 & 53.8 \\
\hline & $\mathrm{b}_{2}-\mathrm{y}_{3}$ & -1439.405167 & 130.6 & 129.3 & 135.8 & -21.7 \\
\hline \multirow{2}{*}{$\mathrm{G}_{5} \mathrm{R}$} & $a_{1}-y_{5}$ & -1647.424114 & 176.9 & 182.3 & 165.5 & 56.3 \\
\hline & $\mathrm{b}_{2}-\mathrm{y}_{4}$ & -1647.446314 & 127.8 & 127.8 & 128.2 & -1.4 \\
\hline
\end{tabular}


Table S2. Relative energies of the transition structures of $a_{1}-y_{N-1}$ and $b_{2}-y_{N-2}$ pathways for each doubly protonated peptides calculated at the M06-2X/6-31+G(d,p) level of theory.

\begin{tabular}{|c|c|c|c|c|c|c|}
\hline $\begin{array}{c}\text { Doubly } \\
\text { Protonated } \\
\text { Peptide }\end{array}$ & Reaction & $E_{\mathrm{e}} / \mathbf{H}$ & $\begin{array}{c}\Delta E_{\mathrm{el}+\mathrm{ZEP}, 0 \mathrm{~K}} \\
\mathrm{~kJ} \mathrm{~mol}^{-1}\end{array}$ & $\begin{array}{c}\Delta H_{298 \mathrm{~K}} \\
\mathrm{~kJ} \mathrm{~mol}^{-1}\end{array}$ & $\begin{array}{c}\Delta G_{298 K} \\
\mathrm{KJ} \mathrm{mol}^{-1}\end{array}$ & $\begin{array}{c}\Delta S_{298 K} \\
\mathrm{~J} \mathrm{~K}^{-1} \mathrm{~mol}^{-1}\end{array}$ \\
\hline \multirow{2}{*}{ AAR } & $a_{1}-y_{2}$ & -1101.455624 & 197.8 & 201.8 & 188.1 & 45.9 \\
\hline & $\mathrm{b}_{2}-\mathrm{y}_{1}$ & -1101.477181 & 151.0 & 148.1 & 156.4 & -28.0 \\
\hline \multirow{2}{*}{$\mathrm{A}_{3} \mathrm{R}$} & $a_{1}-y_{3}$ & -1348.721573 & 184.0 & 190.1 & 168.7 & 71.8 \\
\hline & $\mathrm{b}_{2}-\mathrm{y}_{2}$ & -1348.736257 & 155.5 & 155.7 & 148.9 & 22.6 \\
\hline \multirow{2}{*}{$\mathrm{A}_{4} \mathrm{R}$} & $\mathrm{a}_{1}-\mathrm{y}_{4}$ & -1595.986706 & 192.7 & 197.4 & 182.9 & 48.6 \\
\hline & $\mathrm{b}_{2}-\mathrm{y}_{3}$ & -1596.000576 & 161.7 & 164.6 & 143.6 & 70.4 \\
\hline \multirow{2}{*}{$\mathrm{A}_{5} \mathrm{R}$} & $\mathrm{a}_{1}-\mathrm{y}_{5}$ & -1843.245218 & 194.9 & 198.1 & 188.3 & 33.0 \\
\hline & $\mathrm{b}_{2}-\mathrm{y}_{4}$ & -1843.261538 & 158.3 & 159.3 & 144.3 & 50.6 \\
\hline \multirow{2}{*}{ GGR } & $a_{1}-y_{2}$ & -1022.848354 & 202.6 & 207.8 & 192.0 & 53.1 \\
\hline & $\mathrm{b}_{2}-\mathrm{y}_{1}$ & -1022.872171 & 151.4 & 149.7 & 154.1 & -14.8 \\
\hline \multirow{2}{*}{$\mathrm{G}_{3} \mathrm{R}$} & $a_{1}-y_{3}$ & -1230.810459 & 196.1 & 202.0 & 182.4 & 65.9 \\
\hline & $\mathrm{b}_{2}-\mathrm{y}_{2}$ & -1230.831678 & 154.9 & 153.6 & 154.6 & -3.5 \\
\hline \multirow{2}{*}{$\mathrm{G}_{4} \mathrm{R}$} & $a_{1}-y_{4}$ & -1438.776995 & 206.3 & 210.9 & 198.2 & 42.7 \\
\hline & $\mathrm{b}_{2}-\mathrm{y}_{3}$ & -1438.799364 & 155.1 & 157.0 & 142.5 & 49.1 \\
\hline \multirow{2}{*}{$\mathrm{G}_{5} \mathrm{R}$} & $a_{1}-y_{5}$ & -1646.737525 & 209.5 & 212.9 & 204.9 & 26.9 \\
\hline & $\mathrm{b}_{2}-\mathrm{y}_{4}$ & -1646.754948 & 165.5 & 167.8 & 154.3 & 45.3 \\
\hline
\end{tabular}


Table S3. Relative energies of the consecutive $\mathrm{b}_{2}-\mathrm{a}_{2}$ reaction (Scheme S1) transition structures for each doubly protonated peptides calculated at the B3LYP/6-31+G(d,p) level of theory. The doubly protonated precursor peptide ion GM is the reference value. Note that the $\mathrm{b}_{2}-\mathrm{a}_{2}$ barriers for the $\left[\mathrm{A}_{2} \mathrm{R}+2 \mathrm{H}\right]^{2+}$ and $\left[\mathrm{A}_{3} \mathrm{R}+2 \mathrm{H}\right]^{2+}$ species require less energy than the preceding $\mathrm{b}_{2}-\mathrm{y}_{\mathrm{N}-2}$ transition structures $\left(\Delta \mathrm{E}_{\mathrm{el}+\mathrm{ZEP}, 0 \mathrm{~K}}=144.1\right.$ and $151.9 \mathrm{~kJ} \mathrm{~mol}^{-1}$ respectively, Table $\left.\mathrm{S} 1\right)$.

\begin{tabular}{|c|c|c|c|c|c|}
\hline $\begin{array}{c}\text { Doubly } \\
\text { Protonated } \\
\text { Peptide/ Reaction }\end{array}$ & $E_{\mathrm{el}} / \mathbf{H}$ & $\begin{array}{c}\Delta E_{\mathrm{el}+\mathrm{ZEP}, 0 \mathrm{~K} /} / \\
\mathrm{kJ} \mathrm{mol}^{-1}\end{array}$ & $\begin{array}{l}\Delta H_{298 \mathrm{~K}} / \\
\mathrm{kJ} \mathrm{mol}^{-1}\end{array}$ & $\begin{array}{c}\Delta G_{298 \mathrm{~K}} / \\
\mathrm{kJ} \mathrm{mol}^{-1}\end{array}$ & $\begin{array}{c}\Delta S_{298 \mathrm{~K} /} / \\
\mathrm{J} \mathrm{K}^{-1} \mathrm{~mol}^{-1}\end{array}$ \\
\hline $\mathrm{A}_{2} \mathrm{R} / \mathrm{b}_{2}-\mathrm{a}_{2}$ & -1101.973799 & 90.5 & 92.2 & 36.7 & 186.2 \\
\hline $\mathrm{A}_{3} \mathrm{R} / \mathrm{b}_{2}-\mathrm{a}_{2}$ & -1349.332472 & 97.0 & 101.2 & 38.2 & 211.1 \\
\hline $\mathrm{A}_{4} \mathrm{R} / \mathrm{b}_{2}-\mathrm{a}_{2}$ & -1596.676099 & 149.3 & 152.8 & 93.4 & 199.1 \\
\hline $\mathrm{A}_{5} \mathrm{R} / \mathrm{b}_{2}-\mathrm{a}_{2}$ & -1844.030928 & 156.8 & 158.0 & 104.2 & 180.5 \\
\hline
\end{tabular}

Table S4. Relative energies of the consecutive $\mathrm{b}_{2}-\mathrm{a}_{2}$ reaction (Scheme S1) transition structures for each doubly protonated peptide calculated at the M06-2X/6-31+G(d,p) level of theory. The doubly protonated precursor peptide ion GM is the reference value. Note that the $\mathrm{b}_{2}-\mathrm{a}_{2}$ barriers for the $\left[\mathrm{A}_{2} \mathrm{R}+2 \mathrm{H}\right]^{2+}$ and $\left[\mathrm{A}_{3} \mathrm{R}+2 \mathrm{H}\right]^{2+}$ species require less energy than the preceding $\mathrm{b}_{2}-\mathrm{y}_{\mathrm{N}-2}$ transition structures $\left(\Delta \mathrm{E}_{\mathrm{el}+Z E P, 0 \mathrm{~K}}=151.0\right.$ and $155.5 \mathrm{~kJ} \mathrm{~mol}^{-1}$ respectively, Table S2).

\begin{tabular}{|c|c|c|c|c|c|}
\hline $\begin{array}{l}\text { Doubly Protonated } \\
\text { Peptide/ Reaction }\end{array}$ & $E_{\mathrm{e} 1} / \mathbf{H}$ & $\begin{array}{c}\Delta E_{\text {el+ZEP, oK }} / \\
\mathrm{kJ} \mathrm{mol}^{-1}\end{array}$ & $\begin{array}{l}\Delta H_{298 K} / \\
\mathrm{kJ} \mathrm{mol}^{-1}\end{array}$ & $\begin{array}{l}\Delta G_{298 K} / \\
\mathrm{kJ} \mathrm{mol}^{-1}\end{array}$ & $\begin{array}{c}\Delta S_{298 K} / \\
\mathrm{J} \mathrm{K}^{-1} \mathrm{~mol}^{-1}\end{array}$ \\
\hline $\mathrm{A}_{2} \mathrm{R} / \mathrm{b}_{2}-\mathrm{a}_{2}$ & -1101.480291 & 126.4 & 130.0 & 61.5 & 229.6 \\
\hline $\mathrm{A}_{3} \mathrm{R} / \mathrm{b}_{2}-\mathrm{a}_{2}$ & -1348.736627 & 135.4 & 141.3 & 63.9 & 259.3 \\
\hline $\mathrm{A}_{4} \mathrm{R} / \mathrm{b}_{2}-\mathrm{a}_{2}$ & -1595.986021 & 184.5 & 190.8 & 107.3 & 280.2 \\
\hline$A_{5} R / b_{2}-a_{2}$ & -1843.241845 & 194.1 & 197.8 & 123.0 & 254.8 \\
\hline
\end{tabular}


Table S5. Relative energies of the consecutive $\mathrm{b}_{2}-\mathrm{a}_{2}$ reaction (Scheme S1) transition structures for each doubly protonated peptides calculated at the B3LYP/6-31+G(d,p) level of theory. The doubly protonated precursor peptide ion GM is the reference value. Note that the $\mathrm{b}_{2}-\mathrm{a}_{2}$ barriers for the $\left[\mathrm{G}_{2} \mathrm{R}+2 \mathrm{H}\right]^{2+}$, and $\left[\mathrm{G}_{3} \mathrm{R}+2 \mathrm{H}\right]^{2+}$ species require less energy than the preceding $\mathrm{b}_{2}-\mathrm{y}_{\mathrm{N}-2}$ transition structures $\left(\Delta \mathrm{E}_{\mathrm{el}+\mathrm{ZEP}, 0 \mathrm{~K}}=151.8\right.$, and $159.7 \mathrm{~kJ} \mathrm{~mol}^{-1}$ respectively, Table $\left.\mathrm{S} 1\right)$.

\begin{tabular}{|c|c|c|c|c|c|}
\hline $\begin{array}{c}\text { Doubly Protonated } \\
\text { Peptide/ Reaction }\end{array}$ & $E_{\mathrm{e} 1} / \mathbf{H}$ & $\begin{array}{c}\Delta E_{\text {el+ZEP, } 0 \mathrm{~K} /} \\
\mathrm{kJ} \mathrm{mol}^{-1} \\
\end{array}$ & $\begin{array}{r}\Delta H_{298 \mathrm{~K} /} \\
\mathrm{kJ} \mathrm{mol}^{-1} \\
\end{array}$ & $\begin{array}{r}\Delta G_{298 K} / \\
\mathrm{kJ} \mathrm{mol}^{-1}\end{array}$ & $\begin{array}{c}\Delta S_{298 \mathrm{~K} /} \\
\mathrm{J} \mathrm{K}^{-1} \mathrm{~mol}^{-1} \\
\end{array}$ \\
\hline $\mathrm{G}_{2} \mathrm{R} / \mathrm{b}_{2}-\mathrm{a}_{2}$ & -1023.320914 & 114.7 & 118.0 & 56.4 & 206.6 \\
\hline $\mathrm{G}_{3} \mathrm{R} / \mathrm{b}_{2}-\mathrm{a}_{2}$ & -1231.353657 & 138.9 & 142.5 & 81.2 & 205.6 \\
\hline $\mathrm{G}_{4} \mathrm{R} / \mathrm{b}_{2}-\mathrm{a}_{2}$ & -1439.387993 & 158.3 & 161.6 & 102.1 & 199.6 \\
\hline $\mathrm{G}_{5} \mathrm{R} / \mathrm{b}_{2}-\mathrm{a}_{2}$ & -1647.426223 & 172.3 & 171.7 & 128.8 & 144.1 \\
\hline
\end{tabular}

Table S6. Relative energies of the consecutive $\mathrm{b}_{2}-\mathrm{a}_{2}$ reaction (Scheme S1) transition structures for each doubly protonated peptide calculated at the M06-2X/6-31+G(d,p) level of theory. The doubly protonated precursor peptide ion GM is the reference value. Note that only the $\mathrm{b}_{2}-\mathrm{a}_{2}$ barrier for the $\left[\mathrm{G}_{2} \mathrm{R}+2 \mathrm{H}\right]^{2+}$ requires less energy than the preceding $\mathrm{b}_{2}-\mathrm{y}_{\mathrm{N}-2}$ transition structures $\left(\Delta \mathrm{E}_{\mathrm{el}+\mathrm{ZEP}, 0 \mathrm{~K}}=\right.$ 151.4 and $154.9 \mathrm{~kJ} \mathrm{~mol}^{-1}$ respectively, Table S2).

\begin{tabular}{|c|c|c|c|c|c|}
\hline $\begin{array}{l}\text { Doubly Protonated } \\
\text { Peptide/ Reaction }\end{array}$ & $E_{\mathrm{el}} / \mathbf{H}$ & $\begin{array}{c}\Delta E_{\mathrm{el}+\mathrm{ZEP}, 0 \mathrm{OK}} / \\
\mathrm{kJ} \mathrm{mol}^{-1}\end{array}$ & $\begin{array}{l}\Delta H_{298 \mathrm{~K}} / \\
\mathrm{kJ} \mathrm{mol}^{-1}\end{array}$ & $\begin{array}{l}\Delta G_{298 \mathrm{~K} /} \\
\mathrm{kJ} \mathrm{mol}^{-1}\end{array}$ & $\begin{array}{c}\Delta S_{298 K} / \\
\mathrm{J} \mathrm{K}^{-1} \mathrm{~mol}^{-1}\end{array}$ \\
\hline $\mathrm{G}_{2} \mathrm{R} / \mathrm{b}_{2}-\mathrm{a}_{2}$ & -1022.869735 & 139.0 & 143.6 & 74.0 & 237.1 \\
\hline $\mathrm{G}_{3} \mathrm{R} / \mathrm{b}_{2}-\mathrm{a}_{2}$ & -1230.819582 & 165.5 & 169.8 & 101.0 & 234.4 \\
\hline $\mathrm{G}_{4} \mathrm{R} / \mathrm{b}_{2}-\mathrm{a}_{2}$ & -1438.781067 & 191.2 & 193.4 & 128.7 & 220.4 \\
\hline $\mathrm{G}_{5} \mathrm{R} / \mathrm{b}_{2}-\mathrm{a}_{2}$ & -1646.732275 & 212.0 & 217.0 & 139.4 & 260.0 \\
\hline
\end{tabular}



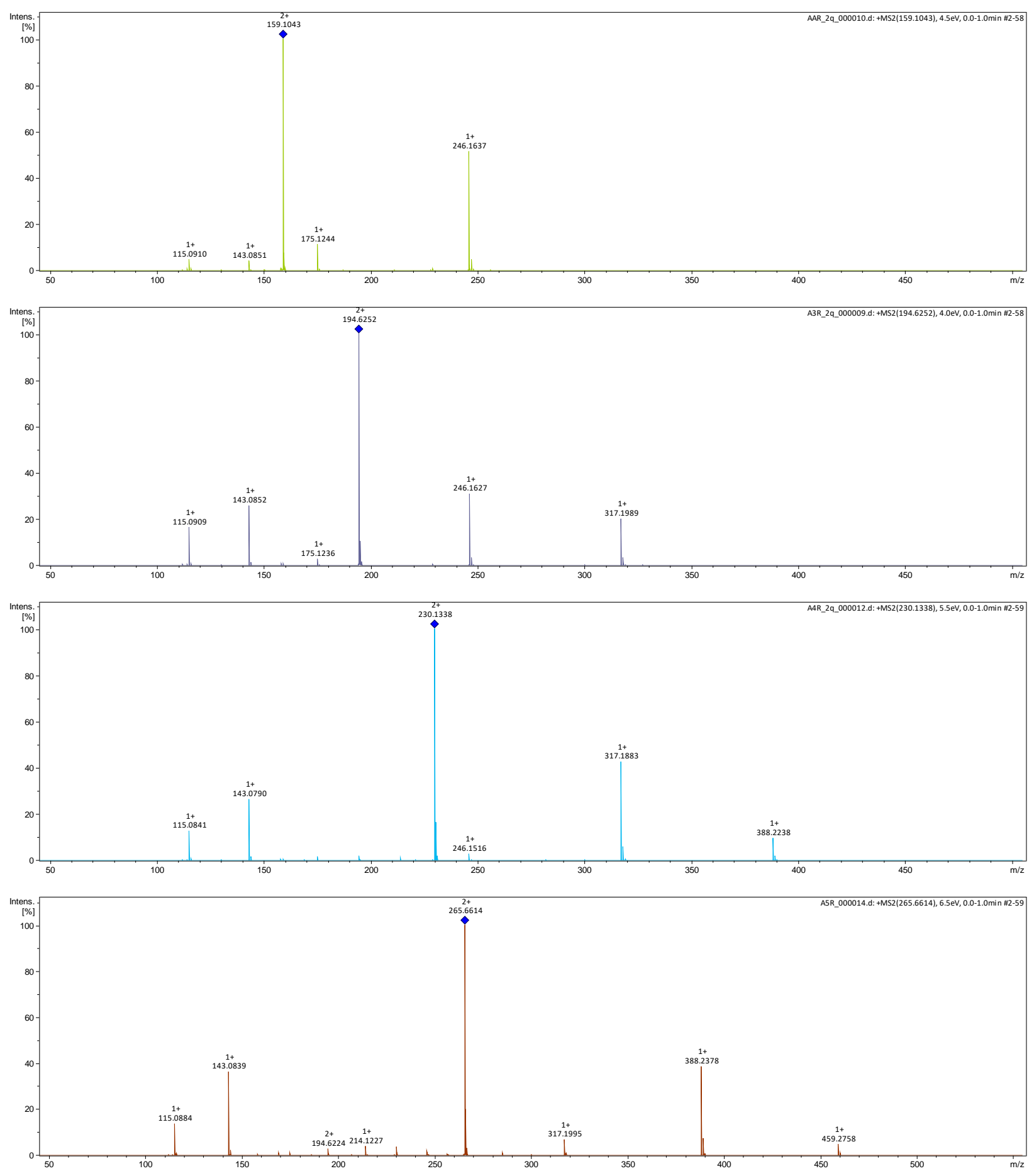

Figure S1. Example $\left[\mathrm{A}_{\mathrm{x}} \mathrm{R}+2 \mathrm{H}\right]^{2+} \mathrm{MS} / \mathrm{MS}$ spectra corresponding to $\sim 50 \%$ dissociation of the precursor ion (indicated by blue diamond) population. 

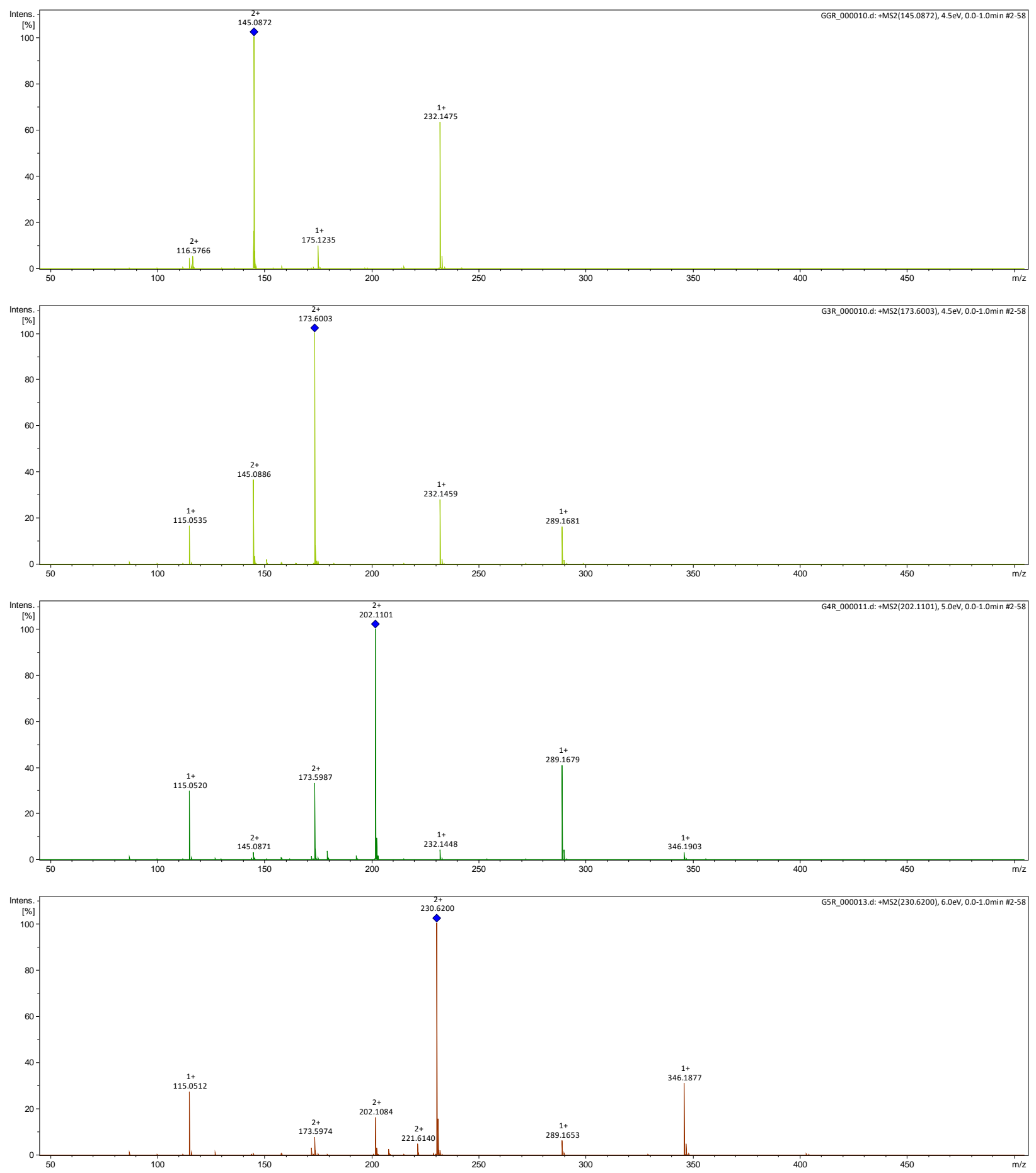

Figure S2. Example $\left[\mathrm{G}_{\mathrm{x}} \mathrm{R}+2 \mathrm{H}\right]^{2+} \mathrm{MS} / \mathrm{MS}$ spectra corresponding to $\sim 50 \%$ dissociation of the precursor ion (indicated by blue diamond) population. 

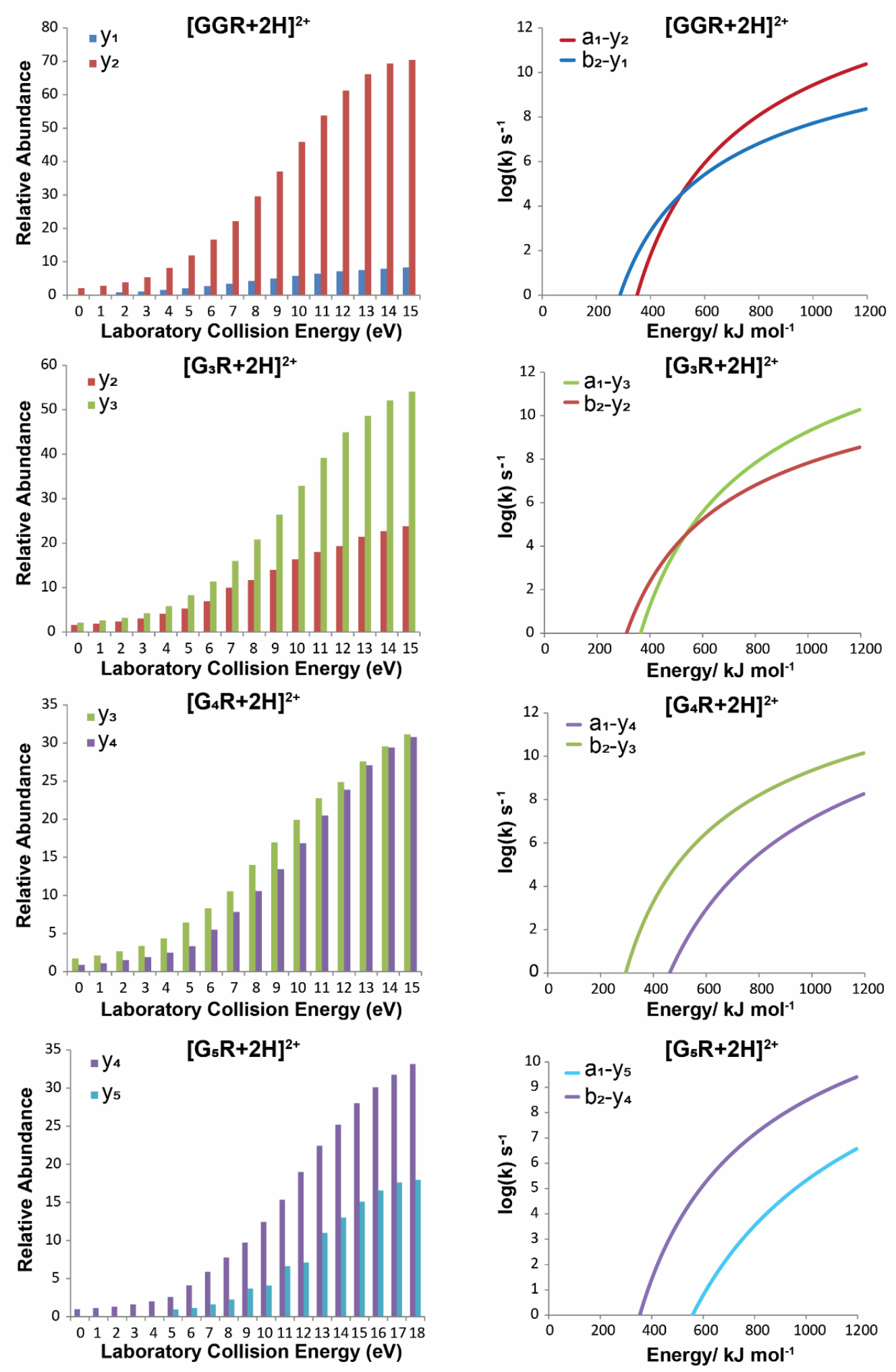

Figure S3. Left column: Relative abundances of $\mathrm{y}_{\mathrm{N}-1}$ and $\mathrm{y}_{\mathrm{N}-2}$ peaks as a function of collision energy for $\left[\mathrm{G}_{\mathrm{x}} \mathrm{R}+2 \mathrm{H}\right]^{2+}$. Right column: RRKM plots for $\mathrm{b}_{2}-\mathrm{y}_{\mathrm{N}-2}$ and $\mathrm{a}_{1}-\mathrm{y}_{\mathrm{N}-1}$ reactions for $\left[\mathrm{G}_{\mathrm{x}} \mathrm{R}+2 \mathrm{H}\right]^{2+}$ using data from M06-2X/6-31+G(d,p) calculations of global minima and TSs. 

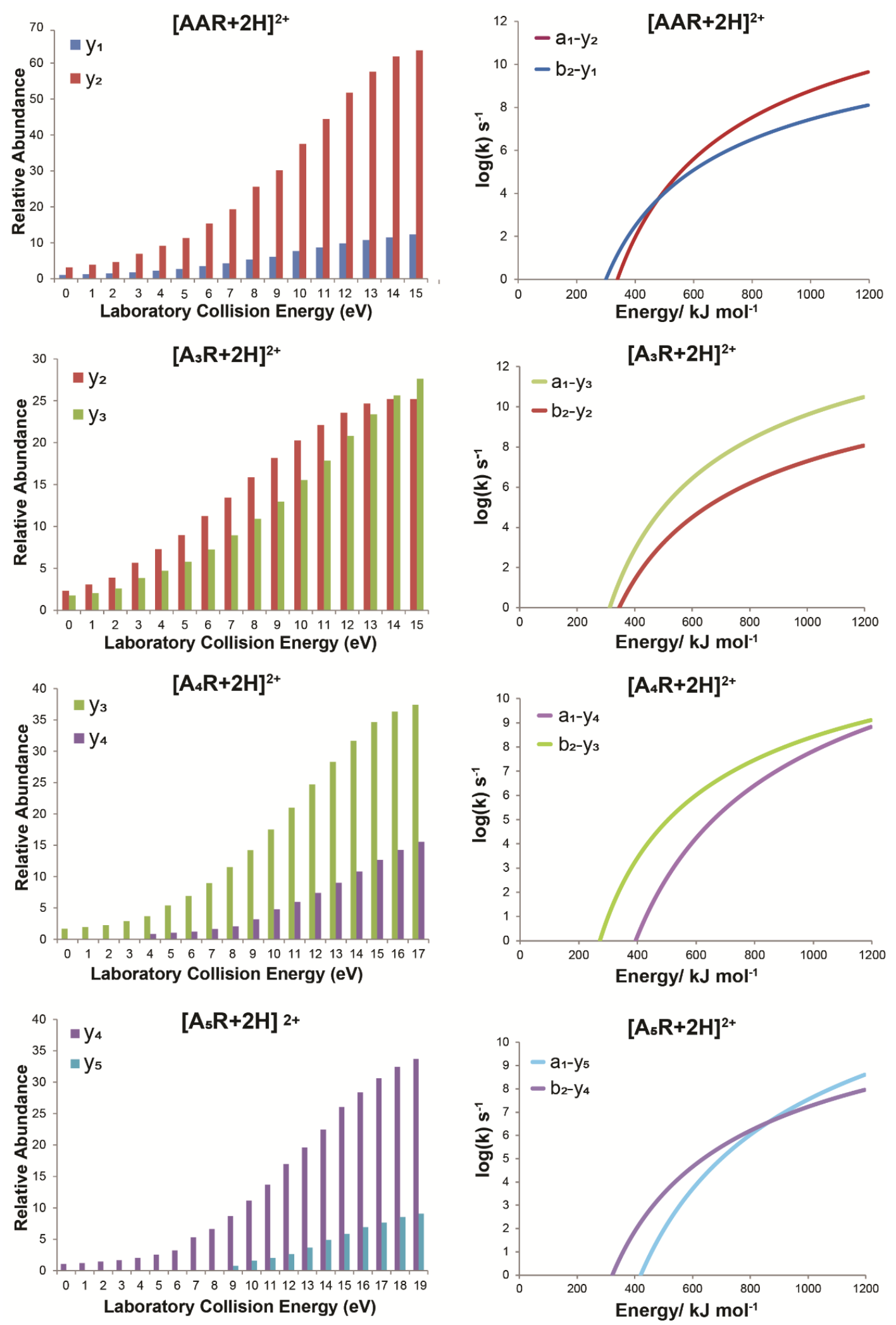

Figure S4. Left column: Relative abundances of $\mathrm{y}_{\mathrm{N}-1}$ and $\mathrm{y}_{\mathrm{N}-2}$ peaks as a function of collision energy for $\left[\mathrm{A}_{x} \mathrm{R}+2 \mathrm{H}\right]^{2+}$. Right column: RRKM plots for $\mathrm{b}_{2}-\mathrm{y}_{\mathrm{N}-2}$ and $\mathrm{a}_{1}-\mathrm{y}_{\mathrm{N}-1}$ reactions for $\left[\mathrm{A}_{\mathrm{x}} \mathrm{R}+2 \mathrm{H}\right]^{2+}$ using data from B3LYP/6-31+G(d,p) calculations of global minima and TSs. 

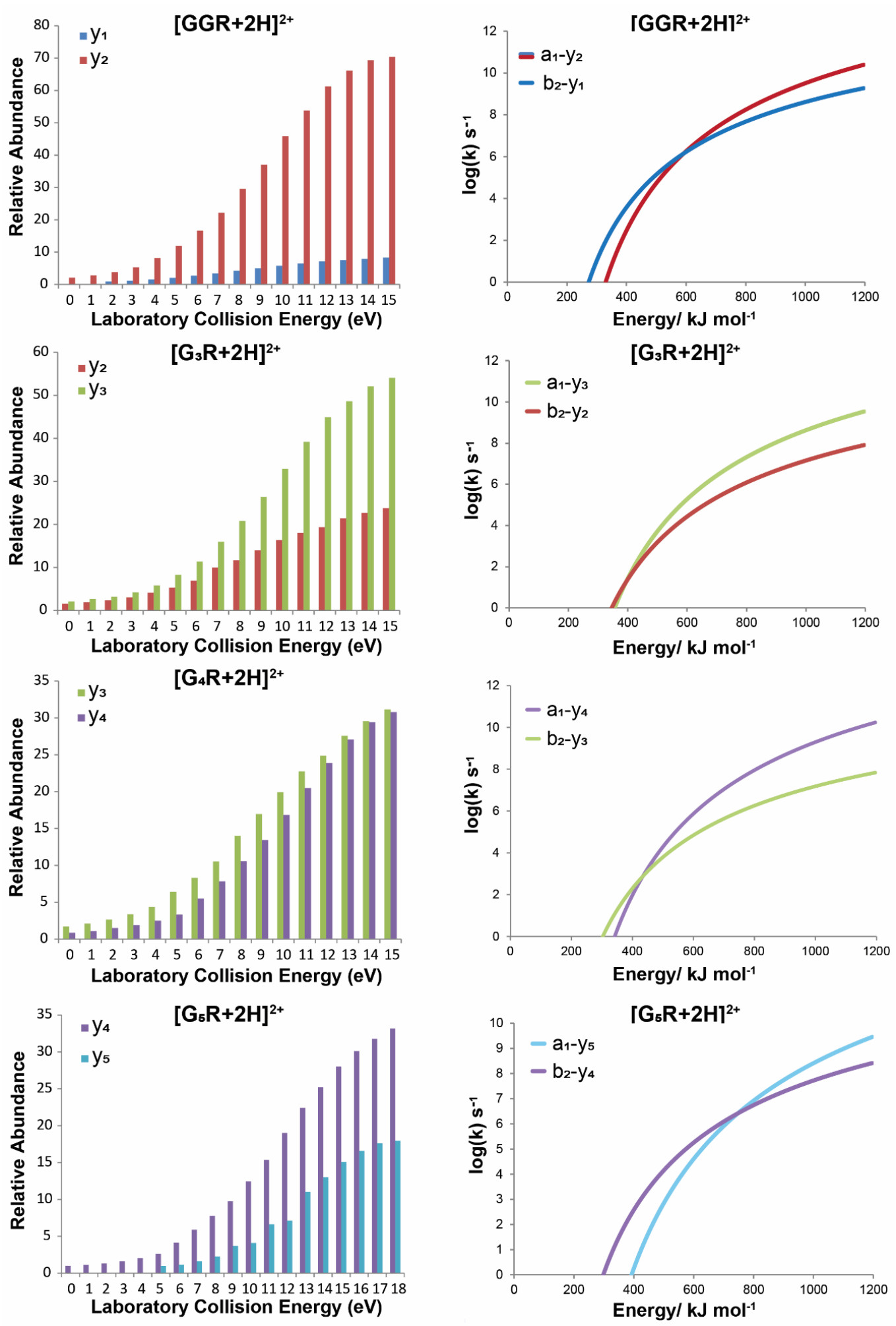

Figure S5. Left column: Relative abundances of $\mathrm{y}_{\mathrm{N}-1}$ and $\mathrm{y}_{\mathrm{N}-2}$ peaks as a function of collision energy for $\left[\mathrm{G}_{\mathrm{x}} \mathrm{R}+2 \mathrm{H}\right]^{2+}$. Right column: RRKM plots for $\mathrm{b}_{2}-\mathrm{y}_{\mathrm{N}-2}$ and $\mathrm{a}_{1}-\mathrm{y}_{\mathrm{N}-1}$ reactions for $\left[\mathrm{G}_{\mathrm{x}} \mathrm{R}+2 \mathrm{H}\right]^{2+}$ using data from B3LYP/6-31+G(d,p) calculations of global minima and TSs. 

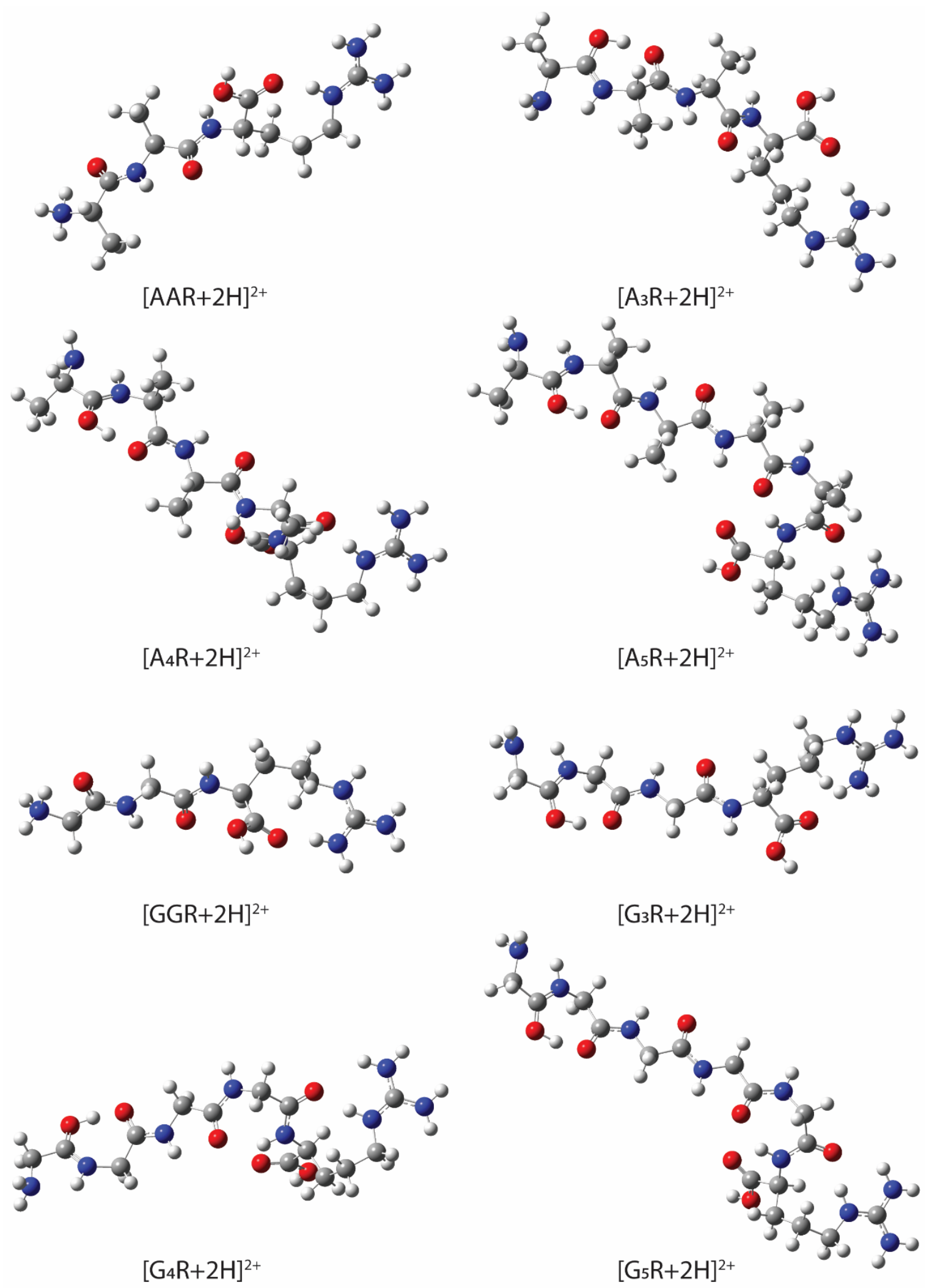

Figure S6. Global minima of doubly protonated peptides calculated at the B3LYP/6-31+G(d,p) level of theory. 


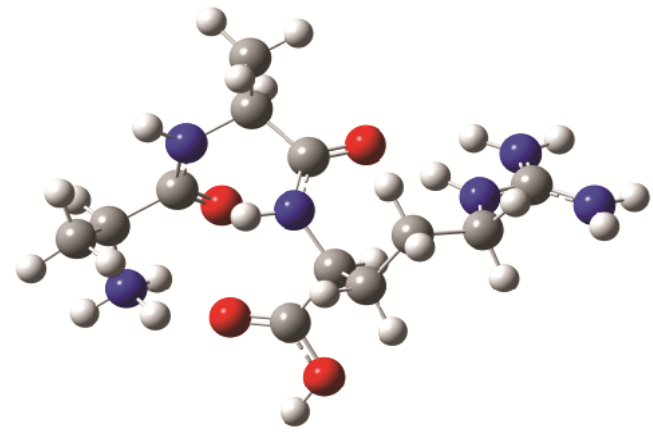

$[\mathrm{AAR}+2 \mathrm{H}]^{2+}$

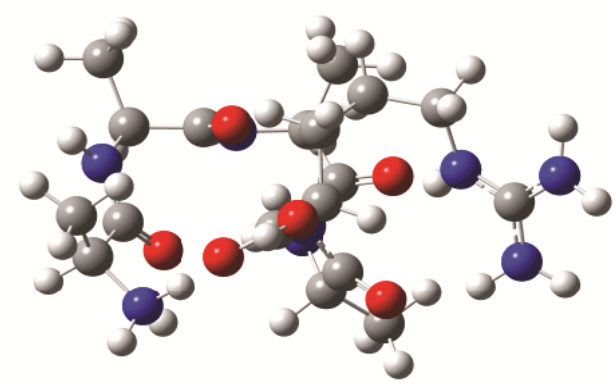

$\left[\mathrm{A}_{4} \mathrm{R}+2 \mathrm{H}\right]^{2+}$

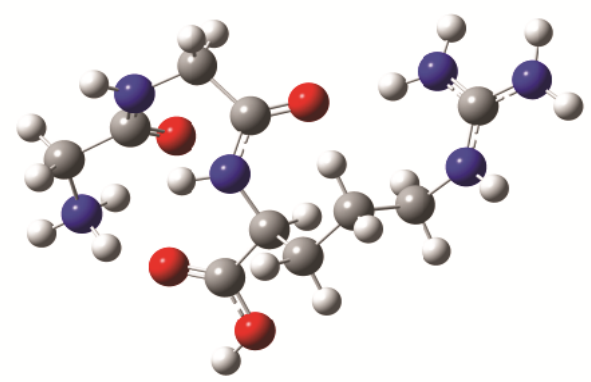

$[\mathrm{GGR}+2 \mathrm{H}]^{2+}$

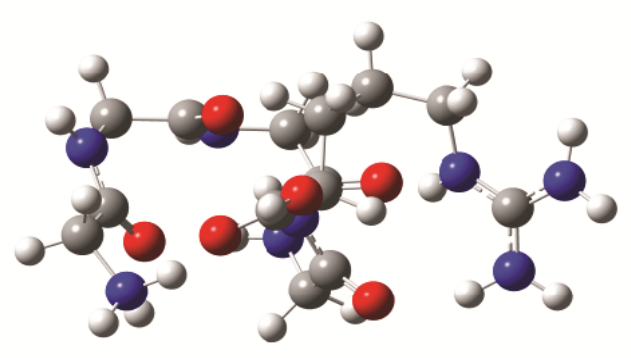

$\left[\mathrm{G}_{4} \mathrm{R}+2 \mathrm{H}\right]^{2+}$

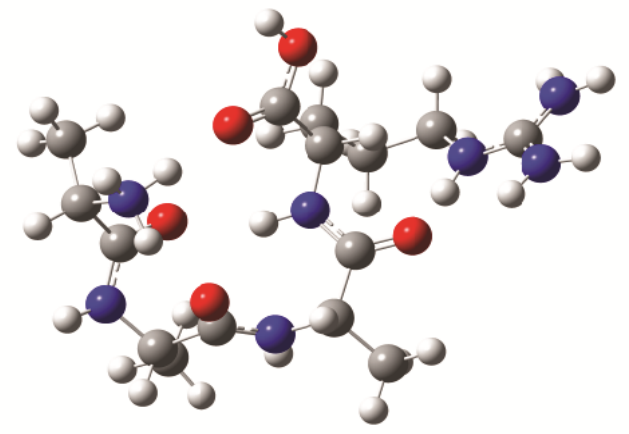

$[\mathrm{A} 3 \mathrm{R}+2 \mathrm{H}]^{2+}$

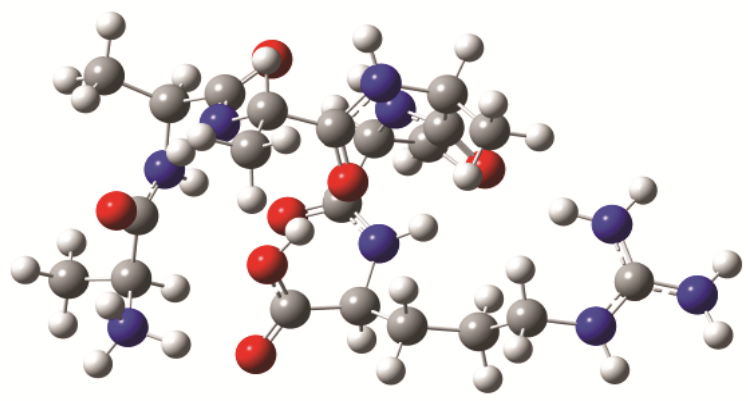

$[\mathrm{A} 5 \mathrm{R}+2 \mathrm{H}]^{2+}$

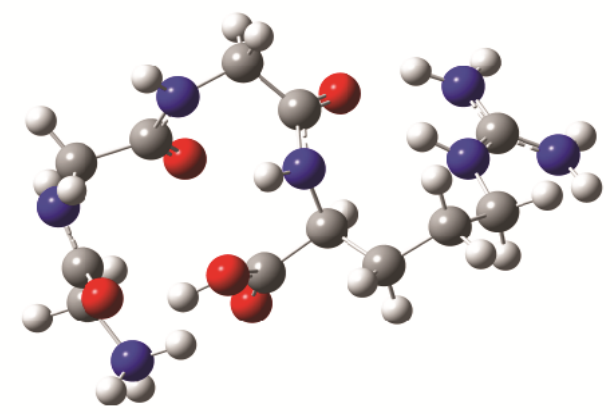

$[\mathrm{G} 3 \mathrm{R}+2 \mathrm{H}]^{2+}$

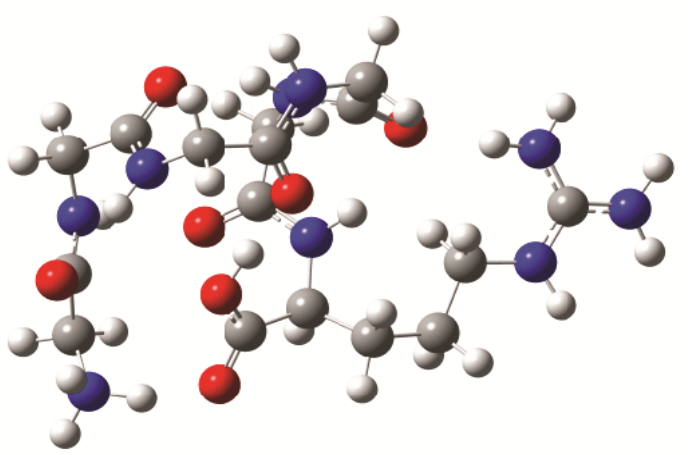

$[\mathrm{G} s \mathrm{R}+2 \mathrm{H}]^{2+}$

Figure S7. Global minima of doubly protonated peptides calculated at the M062X/6-31+G(d,p) level of theory. Note the greater degree of folding relative to the B3LYP congeners. i.e., improved charge-solvation at the cost of decreasing the distances between the charges. 


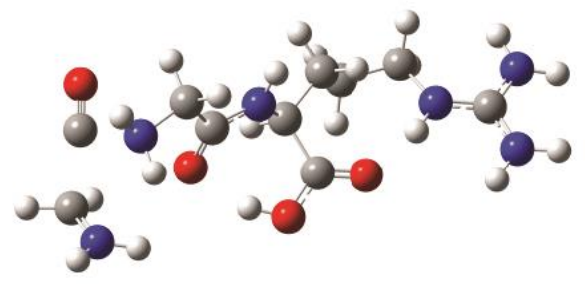

$$
\text { a1-y2 TS }
$$

$[\mathrm{GGR}+2 \mathrm{H}]^{2+}$

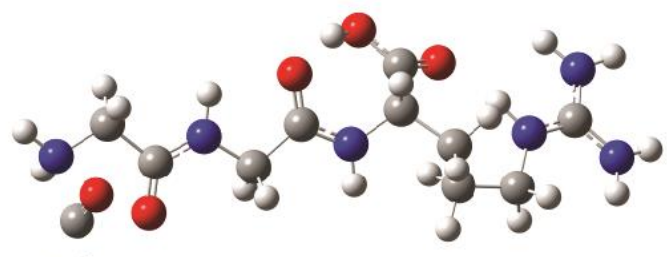

ogs.

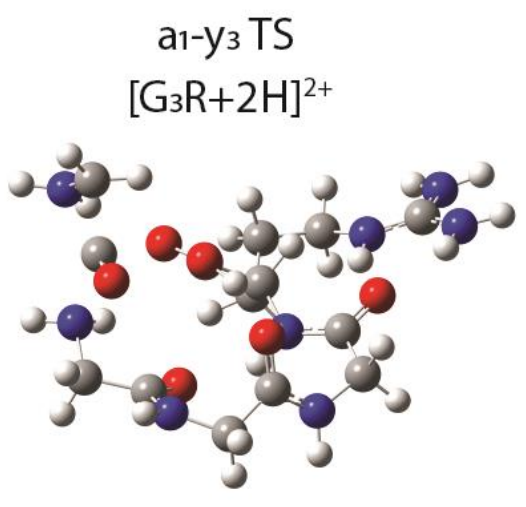

$\mathrm{a}_{1}-\mathrm{y}_{4} \mathrm{TS}$

$\left[\mathrm{G}_{4} \mathrm{R}+2 \mathrm{H}\right]^{2+}$

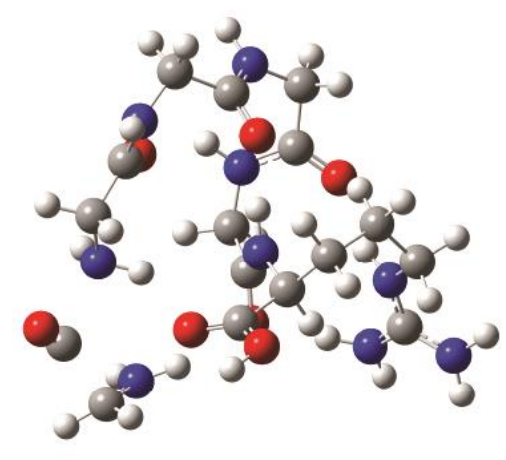

$$
\begin{gathered}
\mathrm{a}_{1}-\mathrm{y}_{5} \mathrm{TS} \\
{[\mathrm{G} \mathrm{R}+2 \mathrm{H}]^{2+}}
\end{gathered}
$$

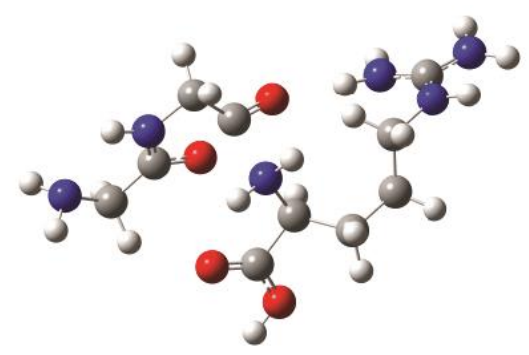

$\mathrm{b}_{2}-\mathrm{y}_{1} \mathrm{TS}$

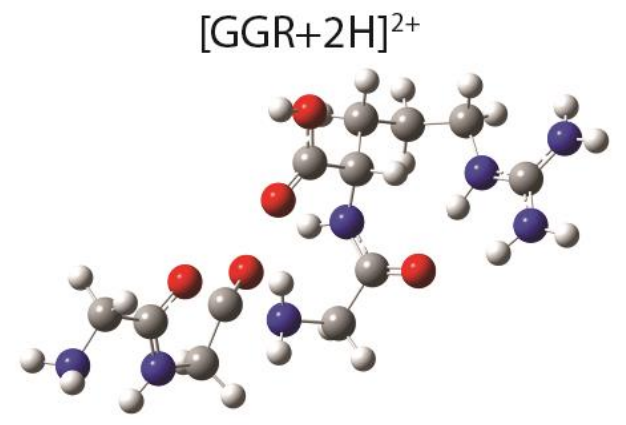

$\mathrm{b}_{2}-\mathrm{y}_{2} \mathrm{TS}$

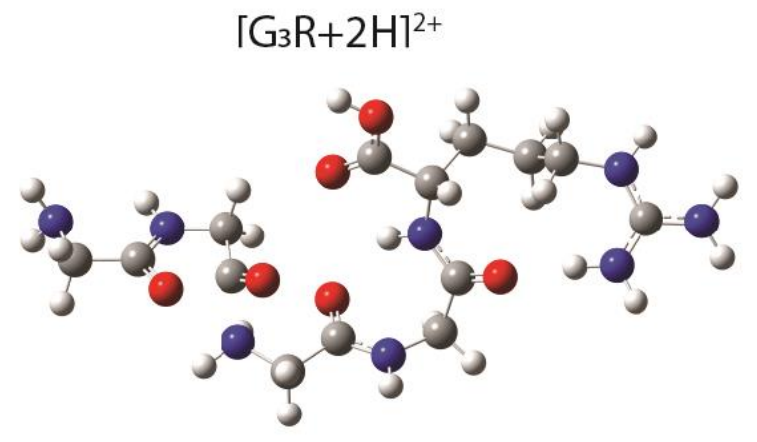

$\mathrm{b}_{2}-\mathrm{y}_{3} \mathrm{TS}$

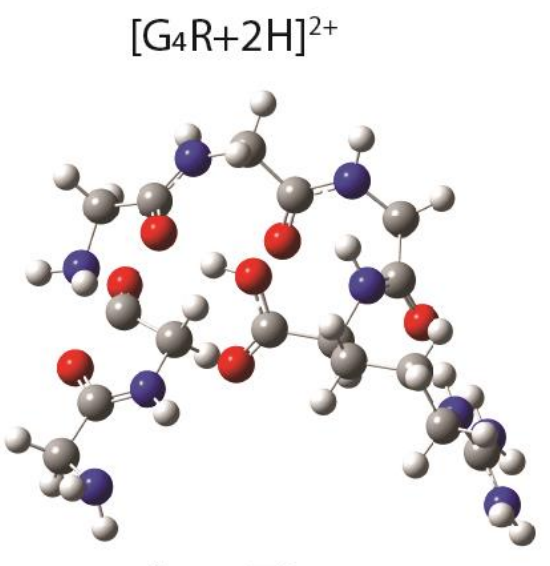

$\mathrm{b}_{2}-\mathrm{y} 4 \mathrm{TS}$

$[\mathrm{G} 5 \mathrm{R}+2 \mathrm{H}]^{2+}$

Figure S8. Transition Structures of $a_{1}-y_{N-1}$ and $b_{2}-y_{N-2}$ reactions for $\left[G_{x} R+H\right]^{+}$calculated at the M06-2X/6-31+G(d,p) level of theory. 

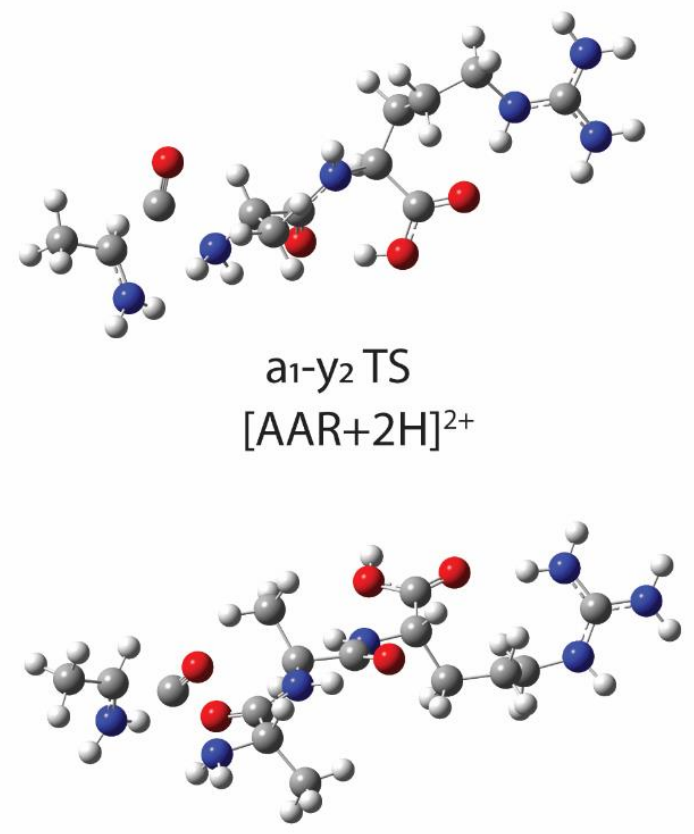

a1- $\mathrm{y}_{3}$ TS

$\left[\mathrm{A}_{3} \mathrm{R}+2 \mathrm{H}\right]^{2+}$

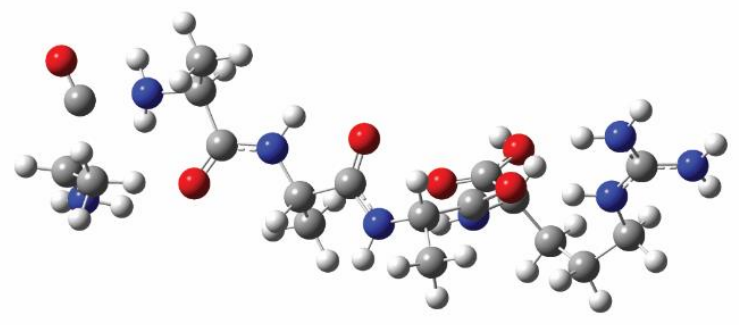

$\mathrm{a}_{1}-\mathrm{y}_{4} \mathrm{TS}$

$\left[\mathrm{A}_{4} \mathrm{R}+2 \mathrm{H}\right]^{2+}$

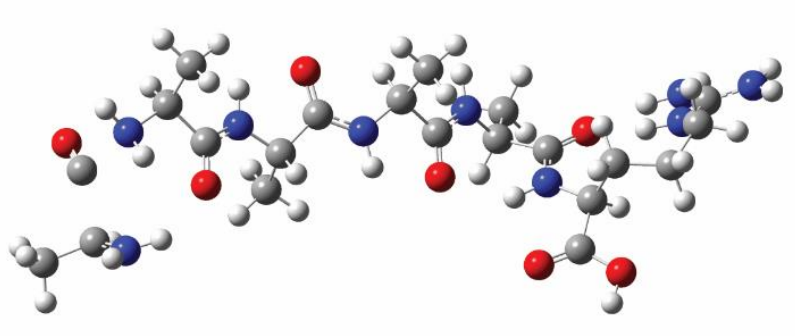

$\mathrm{a}_{1}-\mathrm{y}_{5} \mathrm{TS}$

$\left[\mathrm{A}_{5} \mathrm{R}+2 \mathrm{H}\right]^{2+}$

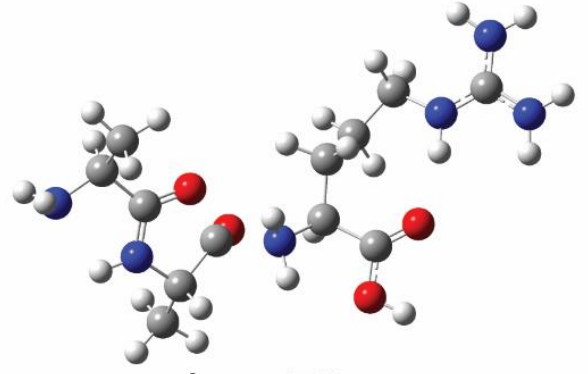

$b_{2}-y_{1} T S$

$[\mathrm{AAR}+2 \mathrm{H}]^{2+}$

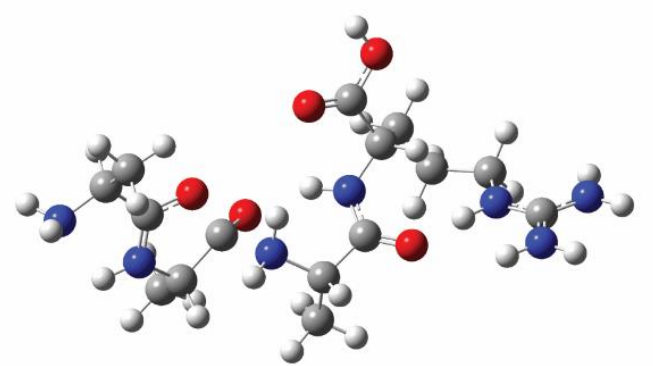

$\mathrm{b}_{2}-\mathrm{y}_{2} \mathrm{TS}$

$\left[\mathrm{A}_{3} \mathrm{R}+2 \mathrm{H}\right]^{2+}$

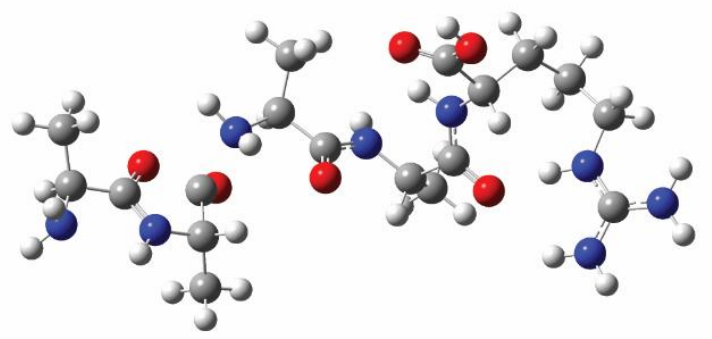

$\mathrm{b}_{2}-\mathrm{y}_{3} \mathrm{TS}$

$\left[\mathrm{A}_{4} \mathrm{R}+2 \mathrm{H}\right]^{2+}$

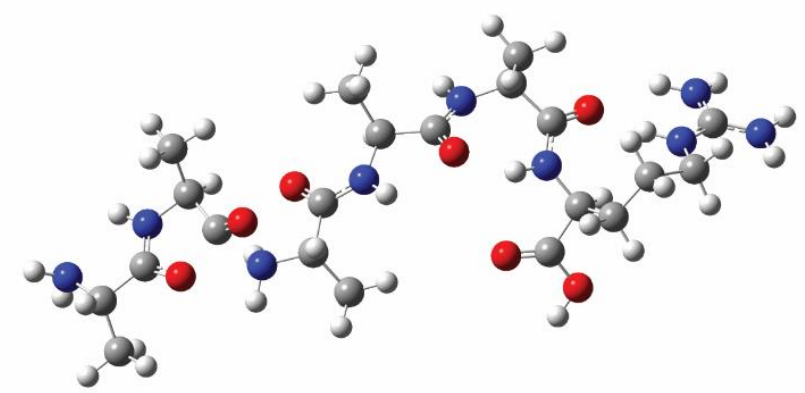

$\mathrm{b}_{2}-\mathrm{y}_{4} \mathrm{TS}$

$\left[\mathrm{A}_{5} \mathrm{R}+2 \mathrm{H}\right]^{2+}$

Figure S9. Transition Structures of $a_{1}-y_{N-1}$ and $b_{2}-y_{N-2}$ reactions for $\left[A_{x} R+H\right]^{+}$calculated at the B3LYP/6-31+G(d,p) level of theory. 

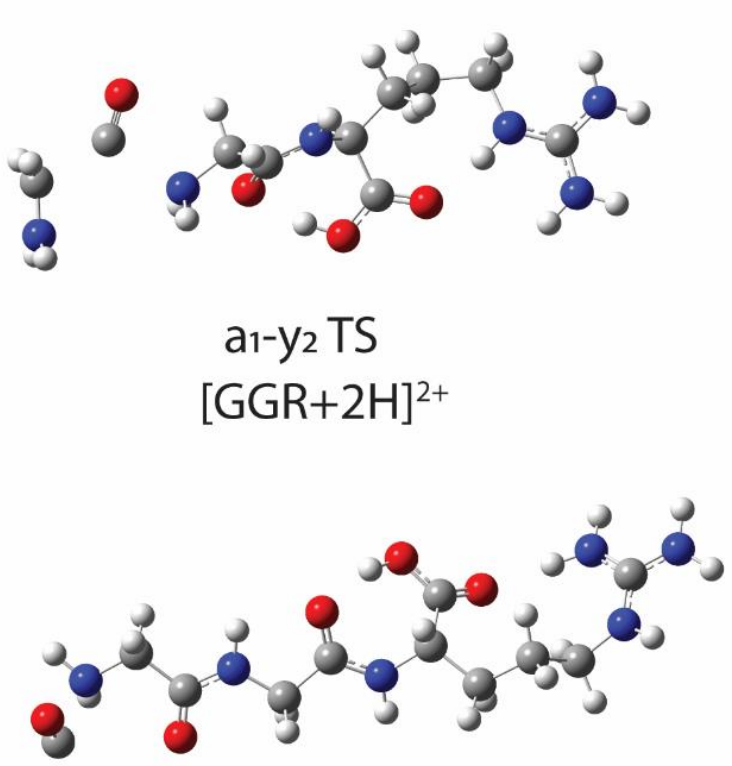

20

$$
\mathrm{a}_{1}-\mathrm{y}_{3} \mathrm{TS}
$$

$[\mathrm{G} 3 \mathrm{R}+2 \mathrm{H}]^{2+}$

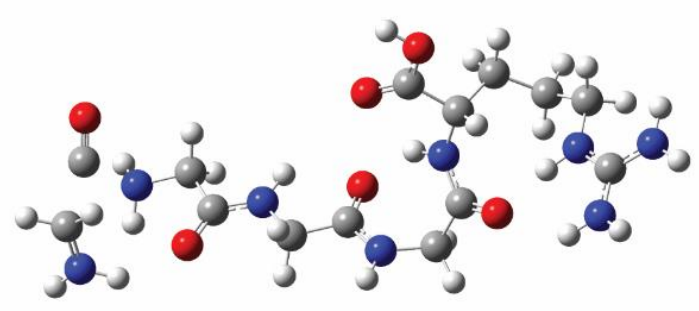

$$
\mathrm{a}_{1}-\mathrm{y} 4 \mathrm{TS}
$$

$\left[\mathrm{G}_{4} \mathrm{R}+2 \mathrm{H}\right]^{2+}$

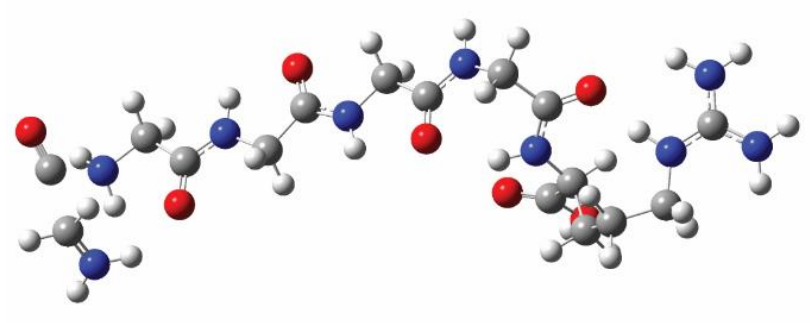

$$
\begin{gathered}
\mathrm{a}_{1}-\mathrm{y}_{5} \mathrm{TS} \\
{\left[\mathrm{G}_{5} \mathrm{R}+2 \mathrm{H}\right]^{2+}}
\end{gathered}
$$

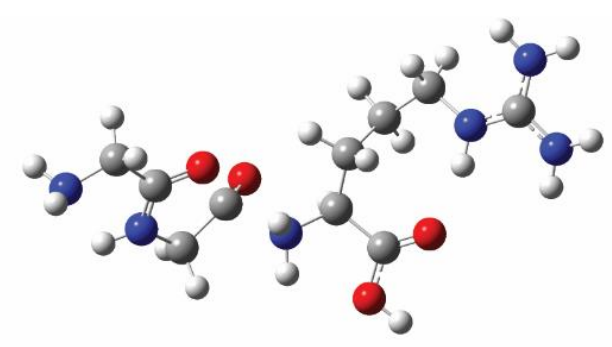

$\mathrm{b}_{2}-\mathrm{y}_{1} \mathrm{TS}$

$[\mathrm{GGR}+2 \mathrm{H}]^{2+}$

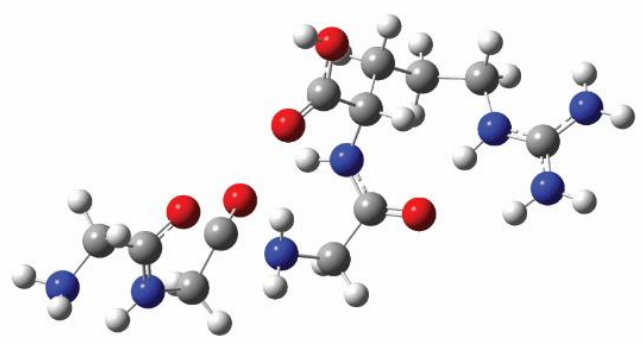

$\mathrm{b}_{2}-\mathrm{y}_{2} \mathrm{TS}$

$\left[\mathrm{G}_{3} \mathrm{R}+2 \mathrm{H}\right]^{2+}$

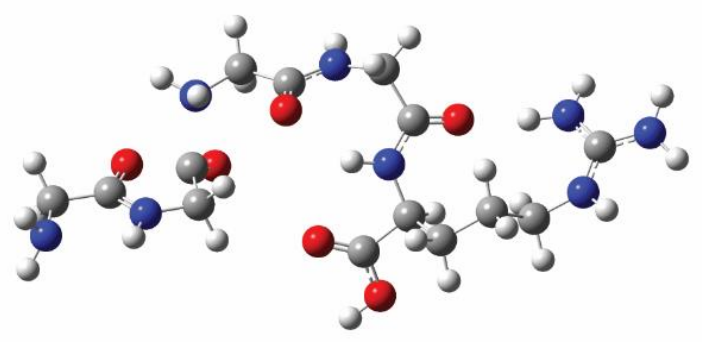

$\mathrm{b}_{2}-\mathrm{y}_{3} \mathrm{TS}$

$\left[\mathrm{G}_{4} \mathrm{R}+2 \mathrm{H}\right]^{2+}$

Figure S10. Transition Structures of $a_{1}-y_{N-1}$ and $b_{2}-y_{N-2}$ reactions for $\left[G_{x} R+H\right]^{+}$calculated at the B3LYP/6-31+G(d,p) level of theory. 

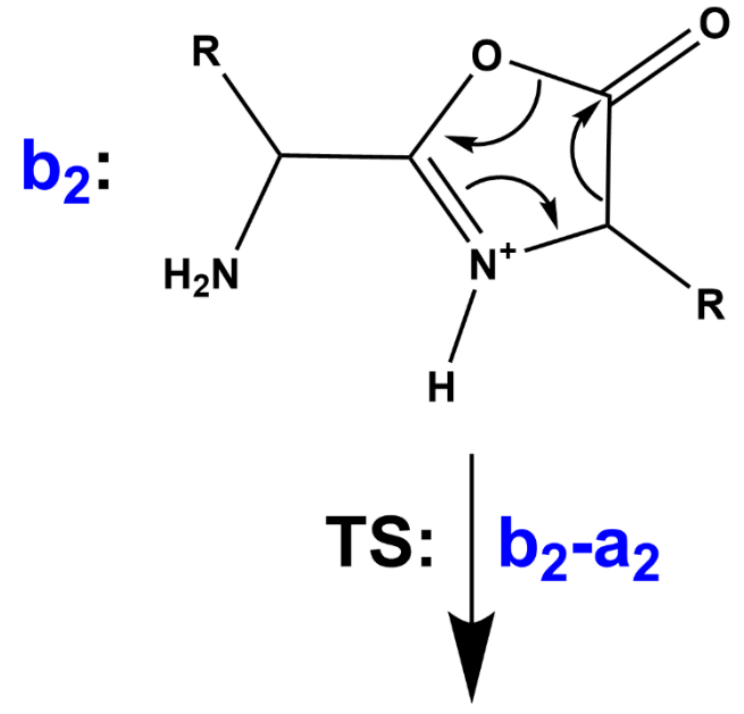
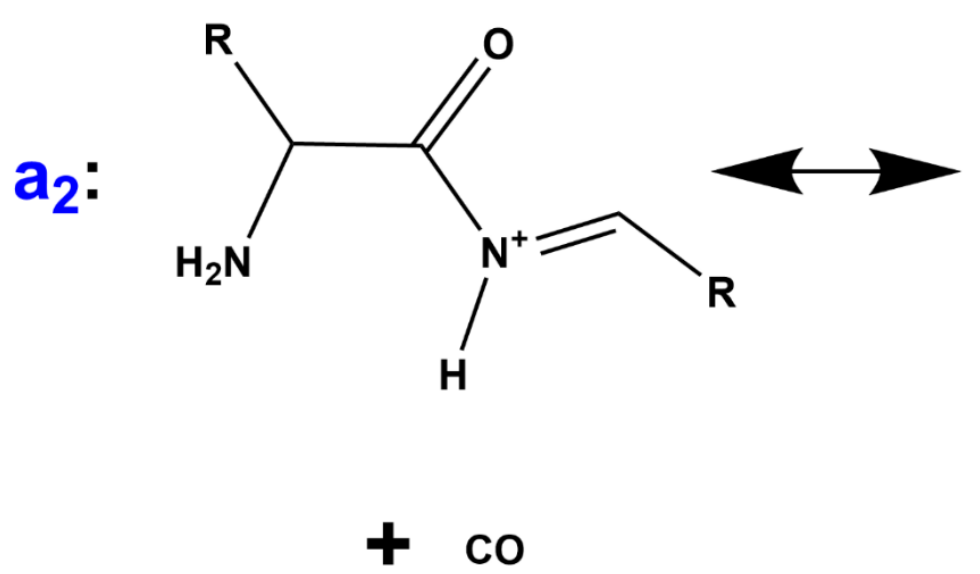

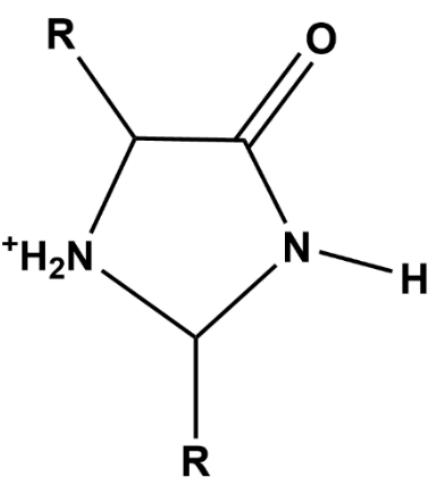

+ co

Scheme S1. Generic $b_{2}-a_{2}$ reaction. Note that the degree of the post-decarbonylation, $a_{2}$ ion cyclization reaction is sequence dependent. 
Images and coordinates of global minima and transition structures discussed in this manuscript.

1. $A A R$

Minimum

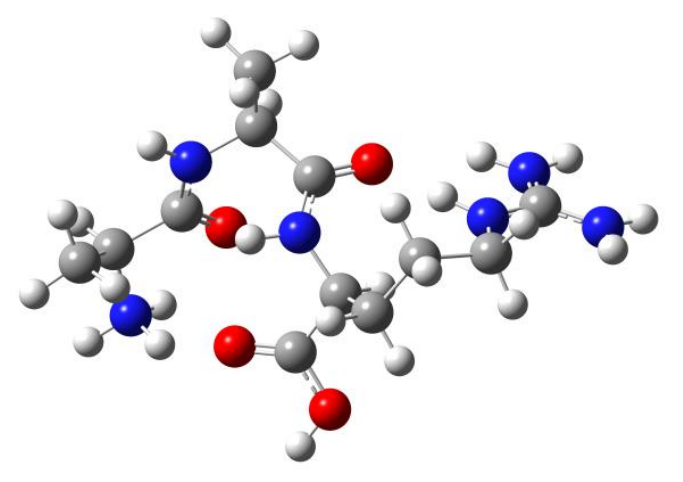

\begin{tabular}{|c|c|c|c|}
\hline $\begin{array}{c}\text { Atom } \\
\text { number }\end{array}$ & $\mathrm{x}$ & $\mathrm{y}$ & $\mathrm{z}$ \\
\hline 7 & 4.147657 & 0.980997 & -1.413521 \\
\hline 1 & 3.708856 & 0.652848 & -2.286604 \\
\hline 1 & 3.498458 & 1.679667 & -1.012452 \\
\hline 1 & 5.055244 & 1.412025 & -1.606888 \\
\hline 6 & 4.256638 & -0.186990 & -0.470295 \\
\hline 1 & 5.018309 & -0.855136 & -0.886629 \\
\hline 6 & 2.885690 & -0.873566 & -0.598974 \\
\hline 8 & 2.214809 & -0.671364 & -1.601610 \\
\hline 7 & 2.457090 & -1.641912 & 0.423422 \\
\hline 6 & 1.146158 & -2.284637 & 0.326719 \\
\hline 1 & 3.061405 & -1.869794 & 1.202336 \\
\hline 1 & 1.104783 & -2.887143 & -0.585988 \\
\hline 6 & 0.047751 & -1.231816 & 0.152578 \\
\hline 8 & -0.985002 & -1.514518 & -0.454631 \\
\hline 7 & 0.257268 & -0.029486 & 0.733674 \\
\hline 6 & -0.442764 & 1.174751 & 0.331404 \\
\hline 1 & 1.163735 & 0.116111 & 1.162027 \\
\hline 1 & -1.065747 & 0.930164 & -0.537152 \\
\hline 6 & 0.602558 & 2.177712 & -0.152962 \\
\hline 8 & 1.806470 & 1.989899 & -0.099721 \\
\hline 6 & -1.301447 & 1.796794 & 1.453687 \\
\hline 1 & -0.629632 & 2.068029 & 2.276587 \\
\hline 1 & -1.733630 & 2.729465 & 1.077464 \\
\hline 6 & -2.406659 & 0.895395 & 2.017673 \\
\hline & & & \\
\hline
\end{tabular}




\begin{tabular}{|l|l|l|l|}
\hline 1 & -2.004587 & -0.093480 & 2.274155 \\
\hline 1 & -2.738862 & 1.337963 & 2.960704 \\
\hline 6 & -3.653432 & 0.727795 & 1.145684 \\
\hline 1 & -4.462272 & 0.314801 & 1.760764 \\
\hline 1 & -3.974075 & 1.703930 & 0.759035 \\
\hline 7 & -3.377249 & -0.175809 & 0.031121 \\
\hline 1 & -2.482001 & -0.674689 & 0.011449 \\
\hline 6 & -4.239494 & -0.527552 & -0.915121 \\
\hline 7 & -5.476941 & -0.025549 & -0.940030 \\
\hline 1 & -6.129972 & -0.270495 & -1.669694 \\
\hline 1 & -5.815840 & 0.569784 & -0.199732 \\
\hline 7 & -3.837303 & -1.380587 & -1.864493 \\
\hline 1 & -4.476837 & -1.749483 & -2.551732 \\
\hline 1 & -2.899800 & -1.762740 & -1.829809 \\
\hline 8 & 0.045286 & 3.278938 & -0.618113 \\
\hline 1 & 0.719806 & 3.925378 & -0.886632 \\
\hline 6 & 4.645418 & 0.294615 & 0.919033 \\
\hline 1 & 4.817659 & -0.555869 & 1.582415 \\
\hline 1 & 5.584975 & 0.852601 & 0.888107 \\
\hline 1 & 3.866959 & 0.932070 & 1.347998 \\
\hline 6 & 0.882307 & -3.152106 & 1.554964 \\
\hline 1 & -0.102575 & -3.614201 & 1.468610 \\
\hline 1 & 1.623647 & -3.952039 & 1.622026 \\
\hline 1 & 0.909483 & -2.559343 & 2.474682 \\
\hline & & & \\
\hline
\end{tabular}

$\mathrm{a}_{1}-\mathrm{y}_{2} \mathrm{TS}:$

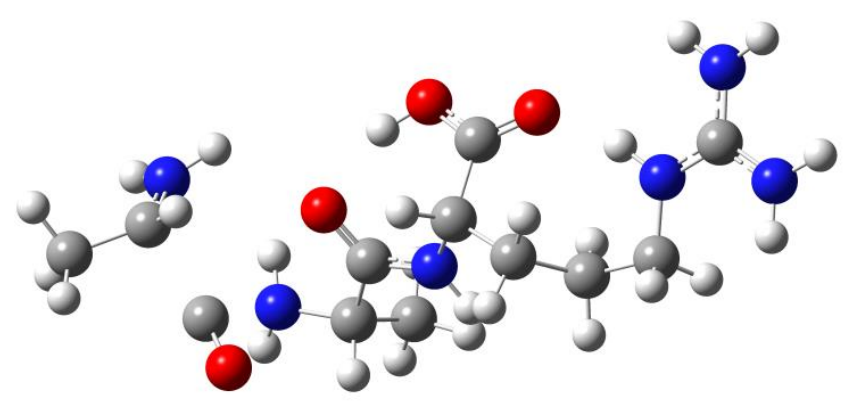

\begin{tabular}{|c|c|c|c|}
\hline $\begin{array}{c}\text { Atom } \\
\text { number }\end{array}$ & $\mathrm{x}$ & $\mathrm{y}$ & $\mathrm{z}$ \\
\hline 7 & -4.842444 & -1.667368 & 0.677827 \\
\hline 6 & -4.929117 & -1.418478 & -0.623931 \\
\hline 6 & -4.349298 & 0.414683 & -1.030595 \\
\hline
\end{tabular}




\begin{tabular}{|c|c|c|c|}
\hline 8 & -3.923729 & 1.116554 & -1.820421 \\
\hline 7 & -3.797619 & 1.273471 & 0.913864 \\
\hline 6 & -2.427503 & 1.751550 & 0.730611 \\
\hline 6 & -1.567852 & 0.547511 & 0.339590 \\
\hline 8 & -2.036115 & -0.601514 & 0.320184 \\
\hline 7 & -0.280344 & 0.790820 & 0.036377 \\
\hline 6 & 0.628321 & -0.227599 & -0.504997 \\
\hline 6 & 1.131645 & -1.178899 & 0.603734 \\
\hline 8 & 2.310158 & -1.366850 & 0.814935 \\
\hline 8 & 0.205584 & -1.809341 & 1.297313 \\
\hline 6 & 1.789160 & 0.415153 & -1.261610 \\
\hline 6 & 2.640847 & 1.400385 & -0.428785 \\
\hline 6 & 4.140548 & 1.257838 & -0.695990 \\
\hline 7 & 4.588496 & -0.038268 & -0.186732 \\
\hline 6 & 5.851699 & -0.441787 & -0.133193 \\
\hline 7 & 6.151950 & -1.589591 & 0.483454 \\
\hline 7 & 6.825469 & 0.289968 & -0.682696 \\
\hline 6 & -1.861279 & 2.468069 & 1.964814 \\
\hline 6 & -6.282557 & -1.449808 & -1.276205 \\
\hline 1 & -5.655710 & -1.607751 & 1.276182 \\
\hline 1 & -3.946797 & -1.877430 & 1.096662 \\
\hline 1 & -4.083053 & -1.783756 & -1.205384 \\
\hline 1 & -3.852288 & 0.600316 & 1.674309 \\
\hline 1 & -4.417013 & 2.051438 & 1.133479 \\
\hline 1 & -2.439219 & 2.440629 & -0.124486 \\
\hline 1 & 0.059997 & 1.742555 & 0.091674 \\
\hline 1 & 0.040991 & -0.844890 & -1.197600 \\
\hline 1 & -0.694041 & -1.600787 & 0.967456 \\
\hline 1 & 2.411417 & -0.407790 & -1.627623 \\
\hline 1 & 1.390076 & 0.915690 & -2.148910 \\
\hline 1 & 2.368496 & 2.434724 & -0.659380 \\
\hline 1 & 2.478683 & 1.255677 & 0.646656 \\
\hline 1 & 4.334750 & 1.327549 & -1.774659 \\
\hline 1 & 4.684812 & 2.060807 & -0.186567 \\
\hline 1 & 3.864754 & -0.642527 & 0.211586 \\
\hline 1 & 7.082869 & -1.978031 & 0.458741 \\
\hline 1 & 5.440376 & -2.113456 & 0.973314 \\
\hline 1 & 7.795869 & 0.028458 & -0.588825 \\
\hline 1 & 6.621347 & 1.113545 & -1.227447 \\
\hline 1 & -0.875589 & 2.900466 & 1.773510 \\
\hline & -2.522471 & 3.289509 & 2.250083 \\
\hline & & & \\
\hline 1 & & & \\
\hline & & & \\
\hline & & &
\end{tabular}




\begin{tabular}{|l|l|l|l|}
\hline 1 & -1.776499 & 1.779058 & 2.810257 \\
\hline 1 & -6.595282 & -2.497213 & -1.335762 \\
\hline 1 & -7.021218 & -0.897837 & -0.690646 \\
\hline 1 & -6.248692 & -1.044669 & -2.288028 \\
\hline
\end{tabular}

$\mathrm{b}_{2}-\mathrm{y}_{1} \mathrm{TS}$ :

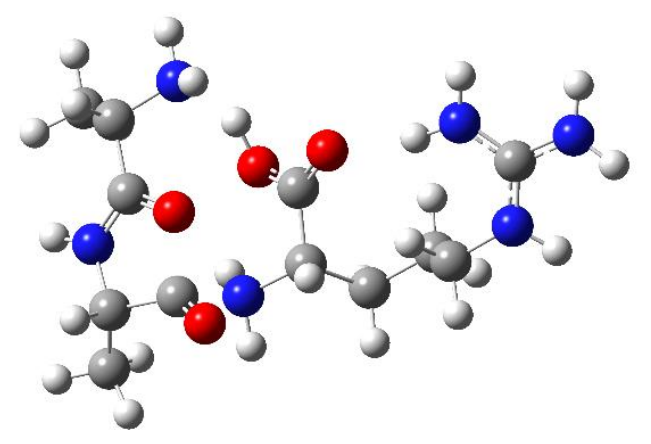

\begin{tabular}{|c|c|c|c|}
\hline $\begin{array}{c}\text { Atom } \\
\text { number }\end{array}$ & $\mathrm{x}$ & $\mathrm{y}$ & $\mathrm{z}$ \\
\hline 7 & 1.728006 & 2.857755 & -0.111415 \\
\hline 6 & 3.054811 & 2.244257 & -0.261948 \\
\hline 6 & 2.880289 & 0.759149 & -0.503975 \\
\hline 8 & 1.852423 & 0.302420 & -1.103530 \\
\hline 7 & 3.777436 & -0.149883 & -0.172497 \\
\hline 6 & 3.465931 & -1.477865 & -0.702155 \\
\hline 6 & 1.986531 & -1.364738 & -1.059982 \\
\hline 8 & 1.315642 & -1.953984 & -1.824956 \\
\hline 7 & 1.061839 & -1.499657 & 0.679941 \\
\hline 6 & -0.318092 & -1.019981 & 0.473391 \\
\hline 6 & -1.319832 & -1.624223 & 1.478370 \\
\hline 6 & -2.743448 & -1.065632 & 1.360339 \\
\hline 6 & -3.365427 & -1.280335 & -0.023486 \\
\hline 7 & -4.714950 & -0.723763 & -0.118567 \\
\hline 6 & -4.990897 & 0.538642 & -0.456481 \\
\hline 7 & -4.012370 & 1.417626 & -0.658669 \\
\hline 7 & -6.265975 & 0.917115 & -0.599625 \\
\hline 6 & -0.325677 & 0.498210 & 0.535098 \\
\hline 8 & -1.138515 & 1.163224 & -0.078518 \\
\hline 8 & 0.614383 & 0.999764 & 1.312896 \\
\hline 1 & 1.198496 & 2.813912 & -0.982249 \\
\hline 1 & 1.835776 & 3.842691 & 0.122903 \\
\hline 1 & 3.570593 & 2.607066 & -1.165570 \\
\hline 1 & 3.968039 & -1.598641 & -1.671594 \\
\hline
\end{tabular}




\begin{tabular}{|c|c|c|c|}
\hline 1 & 1.473160 & -1.035244 & 1.492524 \\
\hline 1 & 1.057914 & -2.506792 & 0.848935 \\
\hline 1 & -0.593060 & -1.296592 & -0.550781 \\
\hline 1 & -0.955740 & -1.446131 & 2.496401 \\
\hline 1 & -1.334267 & -2.710331 & 1.324818 \\
\hline 1 & -2.752629 & 0.001921 & 1.607785 \\
\hline 1 & -3.367382 & -1.561961 & 2.110286 \\
\hline 1 & -3.443129 & -2.346672 & -0.252677 \\
\hline 1 & -2.764409 & -0.828414 & -0.818517 \\
\hline 1 & -5.490208 & -1.312082 & 0.156414 \\
\hline 1 & -4.223015 & 2.329034 & -1.038110 \\
\hline 1 & -3.034300 & 1.216021 & -0.458592 \\
\hline 1 & -6.514445 & 1.885406 & -0.738415 \\
\hline 1 & -7.018423 & 0.244425 & -0.611386 \\
\hline 1 & 0.876427 & 1.927561 & 0.963705 \\
\hline 1 & 4.651141 & 0.070098 & 0.290899 \\
\hline 6 & 3.920696 & 2.538465 & 0.961635 \\
\hline 1 & 4.939528 & 2.161565 & 0.840487 \\
\hline 1 & 4.010540 & 3.619721 & 1.087744 \\
\hline 1 & 3.477929 & 2.120915 & 1.870140 \\
\hline 6 & 3.831961 & -2.624041 & 0.225093 \\
\hline 1 & 3.443505 & -3.559679 & -0.185154 \\
\hline 1 & 4.917841 & -2.720849 & 0.289929 \\
\hline 1 & 3.439671 & -2.474127 & 1.233375 \\
\hline & & & \\
\hline
\end{tabular}

2. $A_{3} R$

minimum

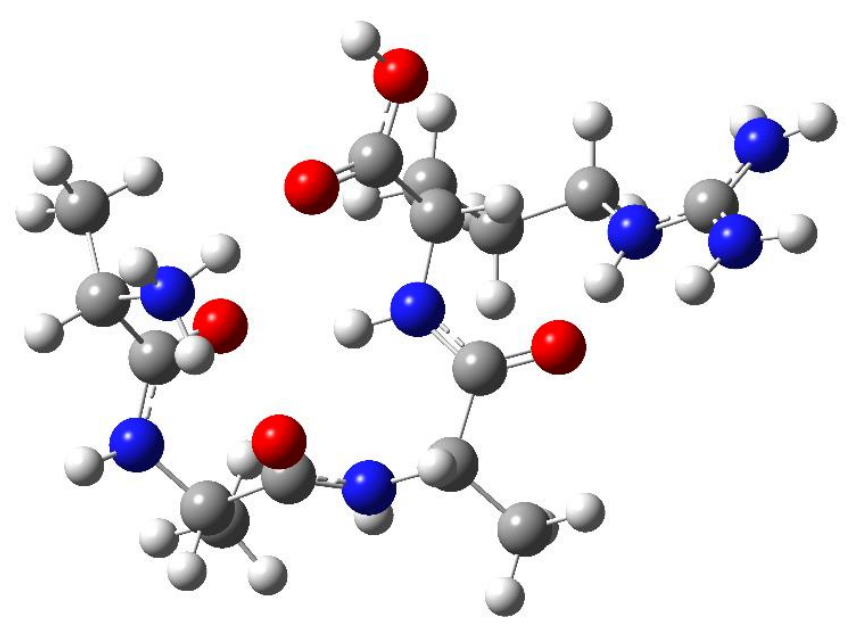




\begin{tabular}{|c|c|c|c|}
\hline $\begin{array}{c}\text { Atom } \\
\text { number }\end{array}$ & $\mathrm{x}$ & $\mathrm{y}$ & $\mathrm{z}$ \\
\hline 7 & -3.424064 & -1.507290 & -1.556809 \\
\hline 1 & -3.385015 & -0.515248 & -1.858939 \\
\hline 1 & -2.432967 & -1.794023 & -1.427745 \\
\hline 1 & -3.846601 & -2.086218 & -2.286740 \\
\hline 6 & -4.154869 & -1.642398 & -0.234043 \\
\hline 1 & -5.215802 & -1.474821 & -0.437659 \\
\hline 6 & -3.538092 & -0.486726 & 0.560302 \\
\hline 8 & -2.380600 & -0.579219 & 0.953144 \\
\hline 7 & -4.242232 & 0.661541 & 0.587028 \\
\hline 6 & -3.514273 & 1.926585 & 0.669227 \\
\hline 1 & -5.174714 & 0.675314 & 0.197693 \\
\hline 1 & -4.188070 & 2.694088 & 0.272918 \\
\hline 6 & -2.359178 & 1.810469 & -0.353452 \\
\hline 8 & -2.508812 & 1.185695 & -1.405655 \\
\hline 7 & -1.192734 & 2.396618 & -0.033942 \\
\hline 6 & -0.046130 & 2.408887 & -0.931562 \\
\hline 1 & -1.108783 & 2.883932 & 0.848472 \\
\hline 1 & -0.438827 & 2.304229 & -1.950037 \\
\hline 6 & 0.879930 & 1.200506 & -0.725247 \\
\hline 8 & 2.059020 & 1.270723 & -1.097142 \\
\hline 7 & 0.364885 & 0.090396 & -0.163671 \\
\hline 6 & 1.128379 & -1.134480 & -0.032844 \\
\hline 1 & -0.607894 & 0.068708 & 0.138303 \\
\hline 1 & 2.060111 & -1.010016 & -0.592858 \\
\hline 6 & 0.396295 & -2.290139 & -0.701656 \\
\hline 8 & -0.738309 & -2.259562 & -1.130992 \\
\hline 6 & 1.428249 & -1.494694 & 1.440336 \\
\hline 1 & 0.466984 & -1.568377 & 1.962773 \\
\hline 1 & 1.885987 & -2.488931 & 1.466571 \\
\hline 6 & 2.318685 & -0.500361 & 2.195214 \\
\hline 1 & 1.931561 & 0.522399 & 2.093383 \\
\hline 1 & 2.251488 & -0.740314 & 3.259948 \\
\hline 6 & 3.805406 & -0.520311 & 1.826978 \\
\hline 1 & 4.375800 & -0.001125 & 2.606202 \\
\hline 1 & 4.162242 & -1.556344 & 1.765880 \\
\hline 7 & 4.015201 & 0.138994 & 0.539739 \\
\hline 1 & 3.246079 & 0.675304 & 0.131607 \\
\hline 7 & 5.119027 & 0.101230 & -0.195856 \\
\hline 7 & 6.231841 & -0.480424 & 0.261331 \\
\hline & & & \\
\hline 7 & & & \\
\hline 1 & & & \\
\hline 1 & & & \\
\hline 1 & & &
\end{tabular}




\begin{tabular}{|l|l|l|l|}
\hline 1 & 7.051264 & -0.575011 & -0.320505 \\
\hline 1 & 6.315941 & -0.760324 & 1.226830 \\
\hline 7 & 5.087483 & 0.632777 & -1.423455 \\
\hline 1 & 5.931524 & 0.790352 & -1.952904 \\
\hline 1 & 4.218458 & 1.021183 & -1.773408 \\
\hline 8 & 1.163973 & -3.369500 & -0.752600 \\
\hline 1 & 0.686247 & -4.102409 & -1.176201 \\
\hline 6 & -3.896818 & -3.007731 & 0.369105 \\
\hline 1 & -4.441734 & -3.098108 & 1.311110 \\
\hline 1 & -4.241337 & -3.802905 & -0.296894 \\
\hline 1 & -2.830506 & -3.131006 & 0.571987 \\
\hline 6 & -3.116612 & 2.290447 & 2.097410 \\
\hline 1 & -2.713788 & 3.306273 & 2.147703 \\
\hline 1 & -4.004891 & 2.272488 & 2.731111 \\
\hline 1 & -2.392348 & 1.578257 & 2.499429 \\
\hline 6 & 0.724168 & 3.717571 & -0.795635 \\
\hline 1 & 1.114268 & 3.841793 & 0.220024 \\
\hline 1 & 1.567740 & 3.726575 & -1.484719 \\
\hline 1 & 0.067751 & 4.557779 & -1.028577 \\
\hline
\end{tabular}

$a_{1}-y_{3} T S$

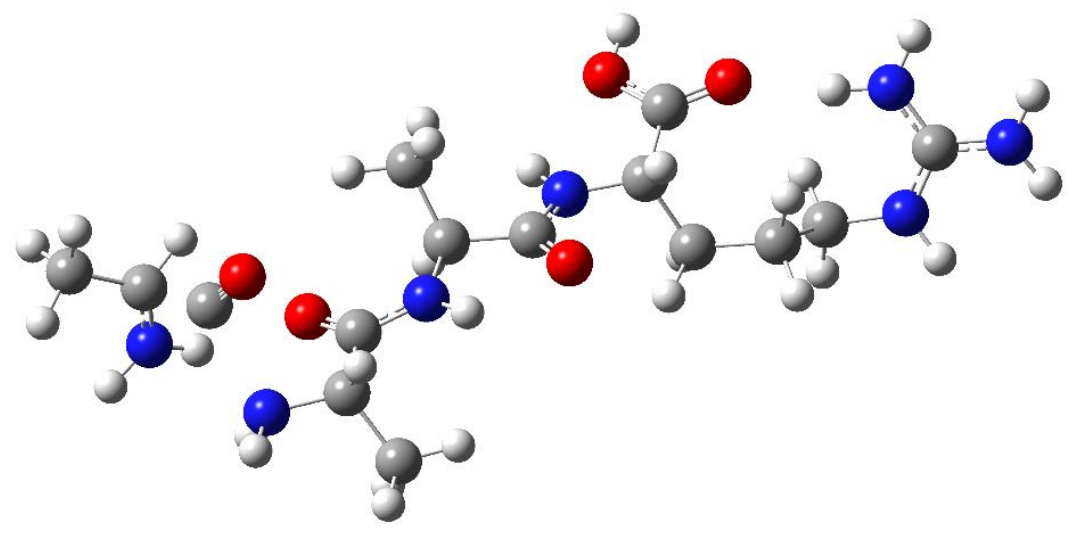

\begin{tabular}{|c|c|c|c|}
\hline $\begin{array}{c}\text { Atom } \\
\text { number }\end{array}$ & $\mathrm{x}$ & $\mathrm{y}$ & $\mathrm{z}$ \\
\hline 7 & 6.632289 & -0.698378 & -1.588622 \\
\hline 6 & 6.690226 & -1.281559 & -0.401190 \\
\hline 6 & 6.125043 & -0.016788 & 1.036160 \\
\hline 8 & 5.675250 & 0.058366 & 2.081637 \\
\hline 7 & 5.559324 & 1.807844 & -0.013481 \\
\hline 6 & 4.144934 & 1.995482 & 0.318047 \\
\hline 6 & 3.393605 & 0.742882 & -0.149858 \\
\hline
\end{tabular}




\begin{tabular}{|c|c|c|c|}
\hline 8 & 3.937894 & -0.101461 & -0.869299 \\
\hline 7 & 2.121318 & 0.638400 & 0.255792 \\
\hline 6 & 1.266874 & -0.469225 & -0.133973 \\
\hline 6 & -0.167852 & -0.028789 & 0.164697 \\
\hline 8 & -0.406344 & 0.961742 & 0.842186 \\
\hline 7 & -1.151696 & -0.821043 & -0.328388 \\
\hline 6 & -2.533373 & -0.549394 & 0.022834 \\
\hline 6 & -3.381604 & -1.802676 & -0.067744 \\
\hline 8 & -4.561859 & -1.827756 & 0.216004 \\
\hline 8 & -2.728410 & -2.872678 & -0.508700 \\
\hline 6 & -3.129675 & 0.599329 & -0.823327 \\
\hline 6 & -4.471863 & 1.149948 & -0.303123 \\
\hline 6 & -5.663457 & 0.723800 & -1.167040 \\
\hline 7 & -6.948236 & 1.155385 & -0.609298 \\
\hline 6 & -7.621409 & 0.491506 & 0.331825 \\
\hline 7 & -7.116919 & -0.613232 & 0.874207 \\
\hline 7 & -8.818735 & 0.941244 & 0.730151 \\
\hline 6 & 1.613718 & -1.750939 & 0.634768 \\
\hline 6 & 3.543386 & 3.264752 & -0.297295 \\
\hline 6 & 8.019042 & -1.747113 & 0.119966 \\
\hline 1 & 5.724287 & -0.529687 & -2.006810 \\
\hline 1 & 7.457841 & -0.298746 & -2.014010 \\
\hline 1 & 5.809350 & -1.872382 & -0.154977 \\
\hline 1 & 6.111923 & 2.596703 & 0.316149 \\
\hline 1 & 5.682668 & 1.729328 & -1.019841 \\
\hline 1 & 4.074175 & 2.045260 & 1.412126 \\
\hline 1 & 1.684098 & 1.344472 & 0.841475 \\
\hline 1 & 1.389168 & -0.639070 & -1.210592 \\
\hline 1 & -0.929759 & -1.645304 & -0.870245 \\
\hline 1 & -2.551945 & -0.233661 & 1.074164 \\
\hline 1 & -3.334502 & -3.632578 & -0.545855 \\
\hline 1 & -2.376920 & 1.390323 & -0.799878 \\
\hline 1 & -3.212619 & 0.270062 & -1.867216 \\
\hline 1 & -4.435789 & 2.243262 & -0.284759 \\
\hline 1 & -4.634539 & 0.832627 & 0.733567 \\
\hline 1 & -5.697016 & -0.360681 & -1.304930 \\
\hline 1 & -5.590115 & 1.167483 & -2.162765 \\
\hline 1 & -7.305622 & 2.059444 & -0.886657 \\
\hline 1 & -7.687685 & -1.189619 & 1.474808 \\
\hline 1 & -6.179444 & -0.950409 & 0.660833 \\
\hline & -9.295182 & 1.680860 & 0.236318 \\
\hline & & & \\
\hline 1 & & & \\
\hline 1 & & &
\end{tabular}




\begin{tabular}{|c|c|c|c|}
\hline 1 & -9.302068 & 0.523240 & 1.510883 \\
\hline 1 & 1.466188 & -1.599995 & 1.707390 \\
\hline 1 & 2.658393 & -2.006813 & 0.448480 \\
\hline 1 & 0.993973 & -2.590426 & 0.309174 \\
\hline 1 & 4.112286 & 4.142265 & 0.019806 \\
\hline 1 & 3.567561 & 3.209621 & -1.389956 \\
\hline 1 & 2.506412 & 3.409841 & 0.015899 \\
\hline 1 & 8.789927 & -0.985577 & -0.016762 \\
\hline 1 & 8.303964 & -2.639214 & -0.446717 \\
\hline 1 & 7.961759 & -2.015164 & 1.175262 \\
\hline
\end{tabular}

$b_{2}-y_{2} T S$

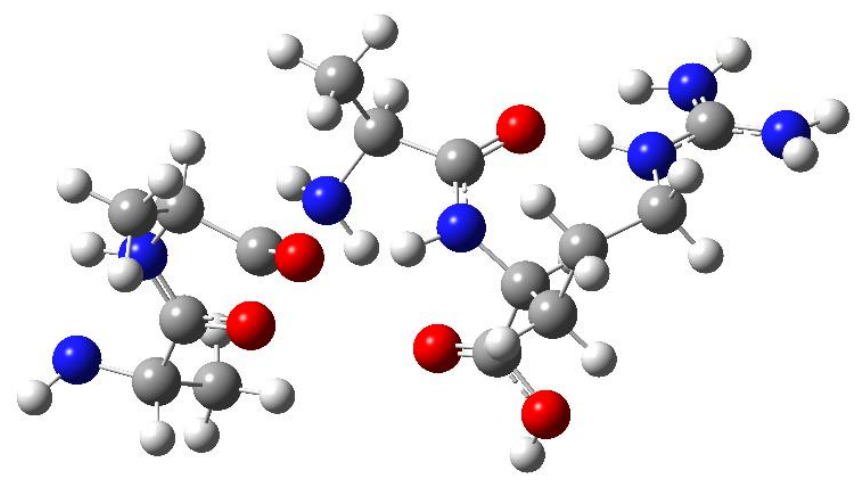

\begin{tabular}{|c|c|c|c|}
\hline $\begin{array}{c}\text { Atom } \\
\text { number }\end{array}$ & $\mathrm{x}$ & $\mathrm{y}$ & $\mathrm{z}$ \\
\hline 7 & 6.340307 & 0.849126 & -0.762536 \\
\hline 1 & 6.650363 & 1.125640 & -1.688169 \\
\hline 1 & 7.077567 & 1.075331 & -0.103927 \\
\hline 6 & 5.065183 & 1.448898 & -0.409592 \\
\hline 1 & 5.139875 & 2.112335 & 0.462008 \\
\hline 6 & 4.139480 & 0.335567 & 0.007153 \\
\hline 8 & 2.947887 & 0.566660 & 0.460450 \\
\hline 7 & 4.454913 & -0.926784 & -0.076156 \\
\hline 6 & 3.423522 & -1.793360 & 0.497692 \\
\hline 1 & 5.399746 & -1.173535 & -0.374101 \\
\hline 1 & 3.130602 & -2.556110 & -0.226137 \\
\hline 6 & 2.279539 & -0.779025 & 0.719475 \\
\hline 8 & 1.441771 & -0.819277 & 1.580340 \\
\hline 7 & 1.370402 & -0.728887 & -1.005134 \\
\hline 6 & 0.178126 & -1.617108 & -1.196844 \\
\hline 1 & 2.025633 & -0.878635 & -1.773668 \\
\hline 1 & 0.047828 & -1.791832 & -2.268595 \\
\hline
\end{tabular}




\begin{tabular}{|c|c|c|c|}
\hline 6 & -1.092632 & -0.870618 & -0.789301 \\
\hline 8 & -2.091340 & -0.917634 & -1.510425 \\
\hline 7 & -1.051729 & -0.164984 & 0.360416 \\
\hline 6 & -1.815503 & 1.059087 & 0.529365 \\
\hline 1 & -0.207289 & -0.244647 & 0.930624 \\
\hline 1 & -2.577378 & 1.090762 & -0.257572 \\
\hline 6 & -0.865878 & 2.220763 & 0.214549 \\
\hline 8 & 0.223531 & 2.075191 & -0.297665 \\
\hline 6 & -2.476648 & 1.164877 & 1.910290 \\
\hline 1 & -1.689291 & 1.174189 & 2.673227 \\
\hline 1 & -2.986123 & 2.131093 & 1.975571 \\
\hline 6 & -3.452197 & 0.027142 & 2.234729 \\
\hline 1 & -2.953831 & -0.945567 & 2.138812 \\
\hline 1 & -3.733275 & 0.119348 & 3.287393 \\
\hline 6 & -4.753526 & 0.009373 & 1.430297 \\
\hline 1 & -5.468561 & -0.659506 & 1.923512 \\
\hline 1 & -5.186399 & 1.017188 & 1.393562 \\
\hline 7 & -4.518839 & -0.453871 & 0.059067 \\
\hline 1 & -3.618774 & -0.860953 & -0.183285 \\
\hline 6 & -5.381410 & -0.349239 & -0.947869 \\
\hline 7 & -6.634946 & 0.058187 & -0.726707 \\
\hline 1 & -7.271911 & 0.227072 & -1.491274 \\
\hline 1 & -7.008990 & 0.116371 & 0.208452 \\
\hline 7 & -4.971971 & -0.630919 & -2.187764 \\
\hline 1 & -3.991456 & -0.829895 & -2.357211 \\
\hline 1 & -5.628207 & -0.737056 & -2.946318 \\
\hline 8 & -1.392391 & 3.395245 & 0.530501 \\
\hline 1 & -0.781043 & 4.109268 & 0.282696 \\
\hline 6 & 4.411767 & 2.221930 & -1.563170 \\
\hline 1 & 4.348467 & 1.590726 & -2.455888 \\
\hline 1 & 3.410097 & 2.562429 & -1.291163 \\
\hline 1 & 5.022990 & 3.094786 & -1.799907 \\
\hline 6 & 3.850127 & -2.421557 & 1.819187 \\
\hline 1 & 4.690063 & -3.101069 & 1.661381 \\
\hline 1 & 4.135611 & -1.653399 & 2.541819 \\
\hline 1 & 3.011852 & -2.987246 & 2.231122 \\
\hline 1 & -0.589359 & -3.552972 & -0.666670 \\
\hline 1 & 1.149874 & -3.534901 & -0.911231 \\
\hline 1 & 0.456797 & -2.851998 & 0.587040 \\
\hline & 1.087336 & 0.260606 & -1.032929 \\
\hline & & & \\
\hline 1 & 0.315810 & -2.967127 & -0.489805 \\
\hline 1 & & & \\
\hline 1 & & & \\
\hline & & &
\end{tabular}


3. $A_{4} R$

Minimum

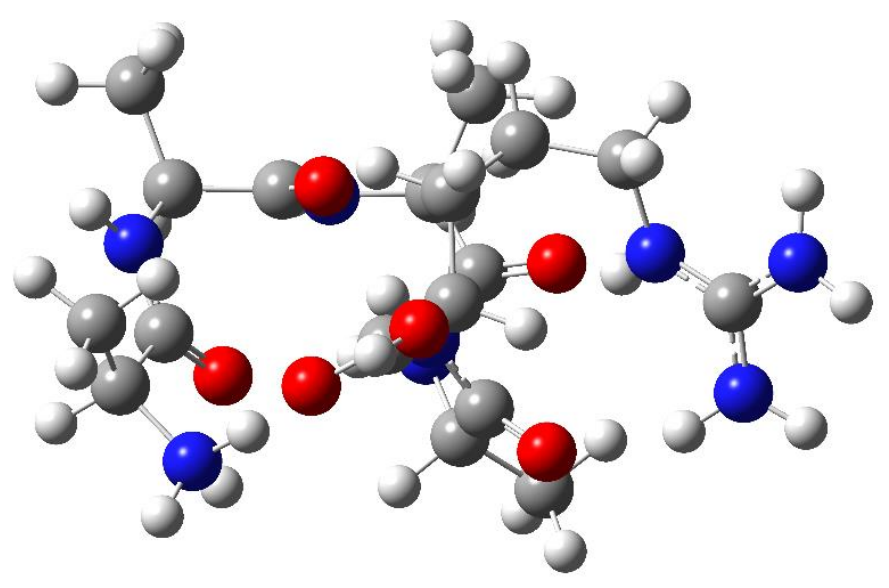

\begin{tabular}{|c|c|c|c|}
\hline $\begin{array}{c}\text { Atom } \\
\text { number }\end{array}$ & $\mathrm{x}$ & $\mathrm{y}$ & $\mathrm{z}$ \\
\hline 7 & -2.488217 & 2.644287 & 1.572022 \\
\hline 1 & -1.621354 & 2.772791 & 0.986136 \\
\hline 1 & -2.701864 & 3.537960 & 2.020842 \\
\hline 1 & -2.298531 & 1.927161 & 2.285123 \\
\hline 6 & -3.626160 & 2.160445 & 0.723959 \\
\hline 1 & -4.541687 & 2.307590 & 1.307492 \\
\hline 6 & -3.403359 & 0.644070 & 0.609195 \\
\hline 8 & -2.748227 & 0.072253 & 1.483045 \\
\hline 6 & -3.689178 & 2.934888 & -0.585473 \\
\hline 1 & -3.697842 & 4.011146 & -0.394909 \\
\hline 1 & -2.835225 & 2.701921 & -1.226395 \\
\hline 1 & -4.616812 & 2.707731 & -1.116088 \\
\hline 7 & -3.967138 & 0.011958 & -0.424372 \\
\hline 6 & -3.788017 & -1.422140 & -0.615118 \\
\hline 1 & -4.413480 & 0.539091 & -1.162756 \\
\hline 1 & -4.292764 & -1.944367 & 0.206211 \\
\hline 6 & -2.287665 & -1.737622 & -0.571741 \\
\hline 8 & -1.456494 & -0.968374 & -1.050407 \\
\hline 7 & -1.950848 & -2.896747 & 0.029465 \\
\hline 6 & -0.561112 & -3.353565 & 0.098300 \\
\hline 1 & -2.680513 & -3.502737 & 0.380268 \\
\hline 1 & -0.553884 & -4.208223 & 0.784374 \\
\hline 6 & 0.320712 & -2.282918 & 0.761732 \\
\hline 8 & 1.509087 & -2.168232 & 0.463339 \\
\hline & & & \\
\hline
\end{tabular}




\begin{tabular}{|l|l|l|l|}
\hline 7 & -0.263314 & -1.514036 & 1.701224 \\
\hline 6 & 0.478536 & -0.494602 & 2.427310 \\
\hline 1 & -1.264561 & -1.566158 & 1.837613 \\
\hline 1 & -0.273475 & 0.079108 & 2.983145 \\
\hline 6 & 1.145963 & 0.509983 & 1.475687 \\
\hline 8 & 2.191256 & 1.071899 & 1.789197 \\
\hline 7 & 0.499840 & 0.786785 & 0.311861 \\
\hline 6 & 1.122754 & 1.635901 & -0.679296 \\
\hline 1 & -0.266639 & 0.183515 & 0.012983 \\
\hline 1 & 2.179743 & 1.743824 & -0.415064 \\
\hline 6 & 0.541100 & 3.039366 & -0.647620 \\
\hline 8 & -0.490937 & 3.373016 & -0.085469 \\
\hline 6 & 0.984514 & 1.064303 & -2.105704 \\
\hline 1 & -0.082338 & 0.980641 & -2.347187 \\
\hline 1 & 1.421520 & 1.788269 & -2.801069 \\
\hline 6 & 1.627408 & -0.310297 & -2.311291 \\
\hline 1 & 1.144996 & -1.055910 & -1.671523 \\
\hline 1 & 1.425560 & -0.612442 & -3.343416 \\
\hline 6 & 3.142390 & -0.373199 & -2.103193 \\
\hline 1 & 3.537339 & -1.268050 & -2.599757 \\
\hline 1 & 3.623138 & 0.508196 & -2.545198 \\
\hline 7 & 3.469852 & -0.411389 & -0.675732 \\
\hline 1 & 2.817687 & -0.914892 & -0.068468 \\
\hline 6 & 4.654324 & -0.086300 & -0.163346 \\
\hline 7 & 4.781766 & 0.035570 & 1.160150 \\
\hline 1 & 3.955611 & 0.262268 & 1.711693 \\
\hline 1 & 5.692149 & 0.132136 & 1.584192 \\
\hline 7 & 5.701765 & 0.139375 & -0.967170 \\
\hline 1 & 5.719020 & -0.216086 & -1.911340 \\
\hline 1 & 6.558583 & 0.530617 & -0.604357 \\
\hline 8 & 1.273076 & 3.888889 & -1.343519 \\
\hline 1 & 0.876034 & 4.776274 & -1.322206 \\
\hline 6 & -4.377911 & -1.842169 & -1.959789 \\
\hline 1 & -4.266047 & -2.918901 & -2.102541 \\
\hline 1 & -5.444804 & -1.608611 & -1.998669 \\
\hline 1 & -3.860888 & -1.332055 & -2.777441 \\
\hline 6 & -0.014572 & -3.790141 & -1.256354 \\
\hline 1 & 1.032130 & -4.079961 & -1.155670 \\
\hline 1 & -0.585076 & -4.645949 & -1.622321 \\
\hline-0.090631 & -2.976276 & -1.980976 \\
\hline 1.483606 & -1.078614 & 3.416518 \\
\hline 1 & & & \\
\hline 1 & & & \\
\hline 1 & & &
\end{tabular}




\begin{tabular}{|l|l|l|l|}
\hline 1 & 1.982442 & -0.276083 & 3.961042 \\
\hline 1 & 0.960472 & -1.718761 & 4.129101 \\
\hline 1 & 2.233957 & -1.675340 & 2.892845 \\
\hline
\end{tabular}

$a_{1}-y_{4} T S$

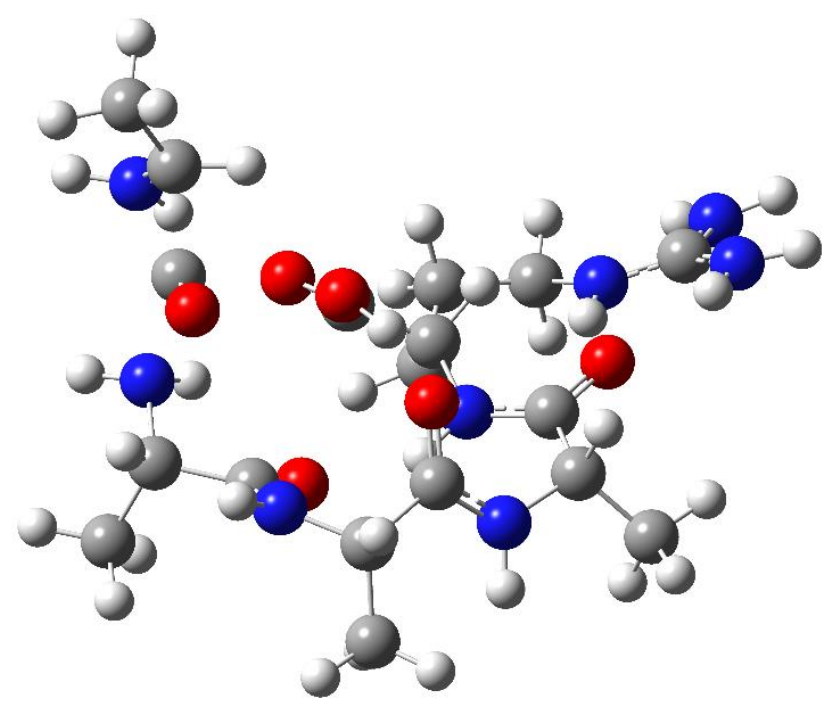

\begin{tabular}{|c|c|c|c|}
\hline $\begin{array}{c}\text { Atom } \\
\text { number }\end{array}$ & $\mathrm{x}$ & $\mathrm{y}$ & $\mathrm{z}$ \\
\hline 7 & -2.586778 & -4.037969 & -0.095912 \\
\hline 6 & -2.715834 & -3.479944 & -1.289685 \\
\hline 6 & -3.530678 & -1.652253 & -1.119623 \\
\hline 8 & -3.978376 & -0.716111 & -1.593578 \\
\hline 7 & -3.382509 & -1.255034 & 1.023970 \\
\hline 6 & -3.898504 & 0.105577 & 1.195400 \\
\hline 6 & -2.810017 & 1.081377 & 0.757788 \\
\hline 8 & -1.658519 & 0.964873 & 1.190917 \\
\hline 7 & -3.191573 & 2.078194 & -0.056110 \\
\hline 6 & -2.325993 & 3.201331 & -0.398383 \\
\hline 6 & -0.968961 & 2.655867 & -0.869298 \\
\hline 8 & -0.904671 & 1.708528 & -1.651010 \\
\hline 7 & 0.136690 & 3.269565 & -0.394654 \\
\hline 6 & 1.476866 & 2.936532 & -0.854993 \\
\hline 6 & 1.907258 & 1.527363 & -0.458099 \\
\hline 8 & 2.991357 & 1.082287 & -0.858554 \\
\hline 7 & 1.064158 & 0.789941 & 0.283497 \\
\hline 6 & 1.264989 & -0.636875 & 0.417372 \\
\hline 6 & -0.040299 & -1.378160 & 0.119687 \\
\hline 8 & -0.341706 & -2.397181 & 0.706652 \\
\hline
\end{tabular}




\begin{tabular}{|c|c|c|c|}
\hline 8 & -0.799455 & -0.931760 & -0.873891 \\
\hline 6 & 1.908943 & -1.075783 & 1.743135 \\
\hline 6 & 2.808691 & -2.315541 & 1.588480 \\
\hline 6 & 4.294295 & -1.987972 & 1.414655 \\
\hline 7 & 4.515788 & -1.051499 & 0.311122 \\
\hline 6 & 5.670237 & -0.859459 & -0.322042 \\
\hline 7 & 5.724080 & 0.050226 & -1.299562 \\
\hline 7 & 6.757546 & -1.574026 & -0.013478 \\
\hline 6 & 2.479630 & 3.974764 & -0.358811 \\
\hline 6 & -2.282466 & 4.224406 & 0.736932 \\
\hline 6 & -4.290874 & 0.429548 & 2.640811 \\
\hline 6 & -3.669480 & -4.082258 & -2.282303 \\
\hline 1 & -1.794330 & -3.782140 & 0.485033 \\
\hline 1 & -3.301354 & -4.649618 & 0.274514 \\
\hline 1 & -1.805740 & -3.019108 & -1.668948 \\
\hline 1 & -3.923575 & -1.912859 & 1.581082 \\
\hline 1 & -2.410457 & -1.309123 & 1.333472 \\
\hline 1 & -4.772644 & 0.217063 & 0.542430 \\
\hline 1 & -4.125335 & 2.059419 & -0.441861 \\
\hline 1 & -2.761631 & 3.663541 & -1.290987 \\
\hline 1 & 0.040179 & 4.034650 & 0.258612 \\
\hline 1 & 1.471525 & 2.913914 & -1.952076 \\
\hline 1 & 0.208924 & 1.196844 & 0.660656 \\
\hline 1 & 1.915146 & -0.927739 & -0.420949 \\
\hline 1 & -0.612653 & -0.010552 & -1.177878 \\
\hline 1 & 2.488710 & -0.229088 & 2.129800 \\
\hline 1 & 1.117458 & -1.271801 & 2.470524 \\
\hline 1 & 2.465871 & -2.928272 & 0.746969 \\
\hline 1 & 2.729923 & -2.946675 & 2.477154 \\
\hline 1 & 4.831058 & -2.917567 & 1.200701 \\
\hline 1 & 4.692289 & -1.560629 & 2.344173 \\
\hline 1 & 3.805590 & -0.350078 & 0.092494 \\
\hline 1 & 6.596276 & 0.292232 & -1.743667 \\
\hline 1 & 4.896847 & 0.595805 & -1.521015 \\
\hline 1 & 6.774924 & -2.188014 & 0.785496 \\
\hline 1 & 7.613069 & -1.477193 & -0.539596 \\
\hline 1 & 2.225728 & 4.961819 & -0.751286 \\
\hline 1 & 3.479454 & 3.713032 & -0.705715 \\
\hline 1 & 2.491841 & 4.016873 & 0.734958 \\
\hline 1 & -1.740836 & 5.128319 & 0.443568 \\
\hline & -3.300574 & 4.533219 & 0.981069 \\
\hline & & & \\
\hline 1 & & & \\
\hline 1 & & &
\end{tabular}




\begin{tabular}{|l|l|l|l|}
\hline 1 & -1.833128 & 3.795211 & 1.637754 \\
\hline 1 & -4.683889 & 1.447160 & 2.717869 \\
\hline 1 & -3.422128 & 0.338166 & 3.297783 \\
\hline 1 & -5.071124 & -0.256381 & 2.981364 \\
\hline 1 & -3.847950 & -3.412356 & -3.123893 \\
\hline 1 & -3.211280 & -5.000017 & -2.664923 \\
\hline 1 & -4.623871 & -4.340043 & -1.817199 \\
\hline
\end{tabular}

$\mathrm{b}_{2}-\mathrm{y}_{3} \mathrm{TS}$

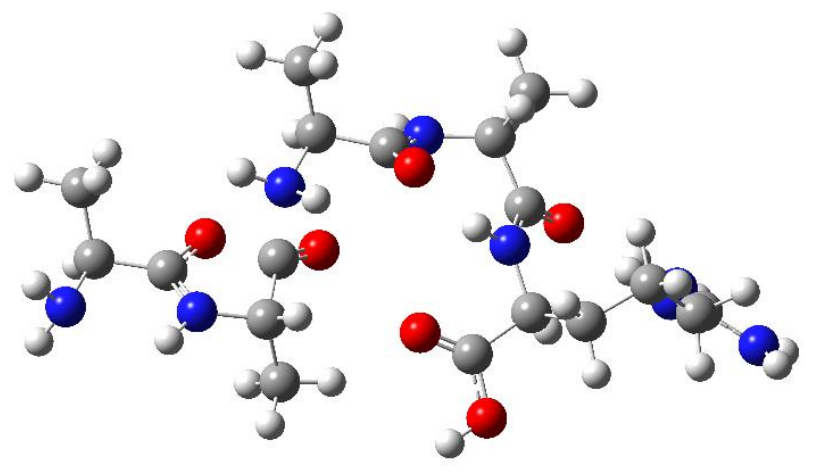

\begin{tabular}{|c|c|c|c|}
\hline $\begin{array}{c}\text { Atom } \\
\text { number }\end{array}$ & $\mathrm{x}$ & $\mathrm{y}$ & $\mathrm{z}$ \\
\hline 7 & -7.112703 & -1.807666 & -0.047301 \\
\hline 6 & -6.492923 & -0.736000 & -0.809978 \\
\hline 1 & -7.856577 & -1.467478 & 0.552724 \\
\hline 1 & -6.653655 & -0.840469 & -1.890779 \\
\hline 6 & -5.000166 & -0.827019 & -0.607066 \\
\hline 8 & -4.171376 & -0.071508 & -1.239116 \\
\hline 7 & -4.455345 & -1.653415 & 0.248507 \\
\hline 6 & -2.995194 & -1.654835 & 0.160385 \\
\hline 1 & -2.533319 & -1.590961 & 1.147317 \\
\hline 6 & -2.738792 & -0.367307 & -0.632282 \\
\hline 8 & -1.802437 & -0.070076 & -1.303368 \\
\hline 7 & -2.966193 & 1.031384 & 0.715399 \\
\hline 6 & -2.332830 & 2.224715 & 0.122770 \\
\hline 1 & -2.447043 & 0.805781 & 1.571596 \\
\hline 1 & -2.544663 & 2.219477 & -0.950976 \\
\hline 6 & -0.836400 & 2.063091 & 0.383873 \\
\hline 8 & -0.476552 & 1.658222 & 1.490356 \\
\hline 7 & 0.002154 & 2.426169 & -0.600846 \\
\hline 6 & 1.459244 & 2.533843 & -0.482782 \\
\hline 1 & -0.396597 & 2.571246 & -1.518671 \\
\hline
\end{tabular}




\begin{tabular}{|c|c|c|c|}
\hline 1 & 1.802865 & 2.961229 & -1.426341 \\
\hline 6 & 2.186917 & 1.186402 & -0.393699 \\
\hline 8 & 3.148488 & 0.970898 & -1.149607 \\
\hline 7 & 1.791419 & 0.328172 & 0.554283 \\
\hline 6 & 2.338493 & -1.003859 & 0.707506 \\
\hline 1 & 0.982609 & 0.584375 & 1.126855 \\
\hline 1 & 2.863008 & -1.278047 & -0.213671 \\
\hline 6 & 1.178115 & -1.977326 & 0.877240 \\
\hline 8 & 0.029201 & -1.657306 & 1.074916 \\
\hline 6 & 3.271937 & -1.135597 & 1.933928 \\
\hline 1 & 2.677268 & -0.889775 & 2.821365 \\
\hline 1 & 3.565751 & -2.186472 & 2.027710 \\
\hline 6 & 4.521076 & -0.246236 & 1.936890 \\
\hline 1 & 4.259436 & 0.794194 & 1.703167 \\
\hline 1 & 4.911061 & -0.235440 & 2.958491 \\
\hline 6 & 5.669089 & -0.700099 & 1.031566 \\
\hline 1 & 6.585729 & -0.173125 & 1.323397 \\
\hline 1 & 5.833914 & -1.778810 & 1.154881 \\
\hline 7 & 5.363289 & -0.397056 & -0.363970 \\
\hline 1 & 4.486517 & 0.104857 & -0.576867 \\
\hline 6 & 6.205152 & -0.512448 & -1.379552 \\
\hline 7 & 5.838206 & -0.030921 & -2.574021 \\
\hline 1 & 4.958837 & 0.463513 & -2.662676 \\
\hline 1 & 6.408978 & -0.148519 & -3.396427 \\
\hline 7 & 7.397360 & -1.099574 & -1.228405 \\
\hline 1 & 7.671715 & -1.502147 & -0.345613 \\
\hline 1 & 8.063258 & -1.144272 & -1.984899 \\
\hline 8 & 1.603432 & -3.239934 & 0.816114 \\
\hline 1 & 0.863172 & -3.842552 & 0.992310 \\
\hline 6 & -6.962503 & 0.657696 & -0.374674 \\
\hline 1 & -6.412870 & 1.440452 & -0.901797 \\
\hline 1 & -8.023348 & 0.768824 & -0.607350 \\
\hline 1 & -6.831107 & 0.784025 & 0.705619 \\
\hline 6 & -2.474423 & -2.858422 & -0.619210 \\
\hline 1 & -1.396884 & -2.747063 & -0.746856 \\
\hline 1 & -2.682012 & -3.779915 & -0.070671 \\
\hline 1 & -2.942109 & -2.915737 & -1.605602 \\
\hline 6 & -2.818946 & 3.524285 & 0.766836 \\
\hline 1 & -2.321545 & 4.382734 & 0.308381 \\
\hline 1 & -3.895686 & 3.644441 & 0.617461 \\
\hline 1 & -2.600993 & 3.527140 & 1.838036 \\
\hline
\end{tabular}




\begin{tabular}{|l|l|l|l|}
\hline 6 & 1.893078 & 3.451499 & 0.665644 \\
\hline 1 & 2.982020 & 3.547168 & 0.661021 \\
\hline 1 & 1.462658 & 4.444821 & 0.521807 \\
\hline 1 & 1.570973 & 3.062048 & 1.632655 \\
\hline 1 & -7.496900 & -2.537266 & -0.637626 \\
\hline 1 & -5.059352 & -2.312675 & 0.738607 \\
\hline 1 & -3.934011 & 1.228083 & 0.969175 \\
\hline
\end{tabular}

\section{$\mathrm{A}_{5} \mathrm{R}$}

Minimum

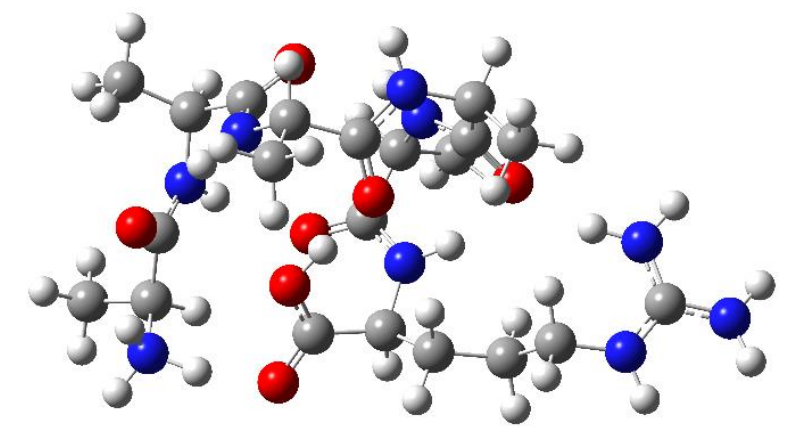

\begin{tabular}{|c|c|c|c|}
\hline $\begin{array}{c}\text { Atom } \\
\text { number }\end{array}$ & $\mathrm{x}$ & $\mathrm{y}$ & $\mathrm{z}$ \\
\hline 7 & -3.878819 & 2.987716 & -1.170613 \\
\hline 1 & -4.388364 & 3.868445 & -1.2643 \\
\hline 1 & -2.850506 & 3.160882 & -1.32756 \\
\hline 1 & -4.238636 & 2.301196 & -1.853197 \\
\hline 6 & -4.037902 & 2.386411 & 0.191003 \\
\hline 1 & -3.17259 & 2.691878 & 0.787562 \\
\hline 6 & -4.037277 & 0.86956 & -0.029427 \\
\hline 8 & -4.402986 & 0.42147 & -1.117795 \\
\hline 7 & -3.700715 & 0.149092 & 1.045938 \\
\hline 6 & -3.767085 & -1.310816 & 1.139648 \\
\hline 1 & -3.131114 & 0.614377 & 1.748383 \\
\hline 1 & -3.575826 & -1.545974 & 2.188687 \\
\hline 6 & -2.615601 & -1.981254 & 0.385738 \\
\hline 8 & -1.795369 & -2.680679 & 0.984328 \\
\hline 7 & -2.541613 & -1.794918 & -0.948216 \\
\hline 6 & -1.549647 & -2.515474 & -1.728903 \\
\hline 1 & -3.225394 & -1.198143 & -1.410176 \\
\hline 1 & -1.705005 & -3.592653 & -1.589038 \\
\hline 6 & -0.153744 & -2.15066 & -1.222641 \\
\hline
\end{tabular}




\begin{tabular}{|c|c|c|c|}
\hline 8 & 0.244148 & -0.974889 & -1.208454 \\
\hline 7 & 0.634621 & -3.145018 & -0.788431 \\
\hline 6 & 1.959828 & -2.889232 & -0.223054 \\
\hline 1 & 0.244466 & -4.076097 & -0.726733 \\
\hline 1 & 2.241478 & -3.795732 & 0.323975 \\
\hline 6 & 1.890994 & -1.743551 & 0.810089 \\
\hline 8 & 2.816567 & -0.913245 & 0.877787 \\
\hline 7 & 0.806319 & -1.719413 & 1.58918 \\
\hline 6 & 0.373935 & -0.648505 & 2.503053 \\
\hline 1 & 0.063421 & -2.382706 & 1.372066 \\
\hline 1 & -0.537925 & -1.028981 & 2.968983 \\
\hline 6 & -0.063048 & 0.613109 & 1.733094 \\
\hline 8 & -1.15992 & 1.144332 & 1.898942 \\
\hline 7 & 0.858695 & 1.085774 & 0.873617 \\
\hline 6 & 0.694594 & 2.220794 & -0.006956 \\
\hline 1 & 1.72161 & 0.556541 & 0.792793 \\
\hline 1 & 0.77417 & 3.166243 & 0.541844 \\
\hline 6 & -0.676501 & 2.259432 & -0.676093 \\
\hline 8 & -1.250868 & 3.32897 & -0.819945 \\
\hline 6 & 1.787536 & 2.118616 & -1.086542 \\
\hline 1 & 1.666557 & 1.142967 & -1.578421 \\
\hline 1 & 1.626002 & 2.892451 & -1.842831 \\
\hline 6 & 3.205129 & 2.24141 & -0.501234 \\
\hline 1 & 3.498394 & 3.295028 & -0.447573 \\
\hline 1 & 3.248526 & 1.856839 & 0.525257 \\
\hline 6 & 4.209075 & 1.465843 & -1.355626 \\
\hline 1 & 3.958848 & 0.399065 & -1.370849 \\
\hline 1 & 4.185856 & 1.809986 & -2.393274 \\
\hline 7 & 5.589418 & 1.610627 & -0.891302 \\
\hline 1 & 6.1056 & 2.41728 & -1.216367 \\
\hline 6 & 6.197288 & 0.819236 & -0.005111 \\
\hline 7 & 5.547853 & -0.17342 & 0.596561 \\
\hline 1 & 6.05828 & -0.812831 & 1.187934 \\
\hline 1 & 4.538318 & -0.324951 & 0.539601 \\
\hline 7 & 7.492377 & 1.025009 & 0.270942 \\
\hline 1 & 7.95787 & 0.521879 & 1.011334 \\
\hline 1 & 8.053982 & 1.662483 & -0.273524 \\
\hline 8 & -1.215301 & 1.156498 & -1.145467 \\
\hline 1 & -0.648085 & 0.323003 & -1.102818 \\
\hline 6 & -5.350446 & 2.833233 & 0.830055 \\
\hline 1 & -5.365534 & 3.917993 & 0.965091 \\
\hline & & & \\
\hline 1 & & & \\
\hline 1 & & &
\end{tabular}




\begin{tabular}{|c|c|c|c|}
\hline 1 & -6.203951 & 2.531281 & 0.215653 \\
\hline 1 & -5.457736 & 2.373126 & 1.813925 \\
\hline 6 & -5.130469 & -1.879905 & 0.74468 \\
\hline 1 & -5.133394 & -2.958253 & 0.920294 \\
\hline 1 & -5.905648 & -1.430256 & 1.369307 \\
\hline 1 & -5.368373 & -1.68917 & -0.302324 \\
\hline 6 & -1.676737 & -2.146646 & -3.203904 \\
\hline 1 & -0.939684 & -2.689558 & -3.799489 \\
\hline 1 & -2.67155 & -2.411825 & -3.568932 \\
\hline 1 & -1.516261 & -1.073682 & -3.340912 \\
\hline 6 & 2.99142 & -2.605891 & -1.309328 \\
\hline 1 & 3.976934 & -2.446259 & -0.867923 \\
\hline 1 & 3.048849 & -3.45872 & -1.98806 \\
\hline 1 & 2.70264 & -1.718885 & -1.879355 \\
\hline 6 & 1.397346 & -0.319181 & 3.590385 \\
\hline 1 & 0.973953 & 0.42076 & 4.273779 \\
\hline 1 & 1.629256 & -1.222476 & 4.158527 \\
\hline 1 & 2.32132 & 0.076478 & 3.166192 \\
\hline 1 & 2.32132 & 0.076478 & 3.166192 \\
\hline & & &
\end{tabular}

$a_{1}-y_{5} T S$

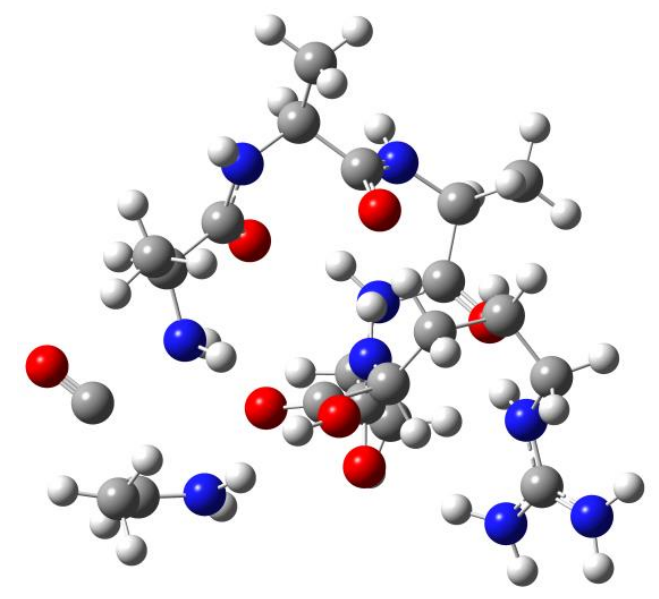

\begin{tabular}{|c|c|c|c|}
\hline $\begin{array}{c}\text { Atom } \\
\text { number }\end{array}$ & $\mathrm{x}$ & $\mathrm{y}$ & $\mathrm{z}$ \\
\hline 7 & -2.268667 & -3.202977 & -1.130082 \\
\hline 1 & -1.860162 & -3.154468 & -2.053203 \\
\hline 1 & -1.658985 & -2.986523 & -0.344950 \\
\hline 6 & -3.551165 & -3.465813 & -0.947239 \\
\hline 1 & -4.082818 & -3.848548 & -1.815528 \\
\hline 6 & -4.626442 & -1.706735 & -0.976816 \\
\hline
\end{tabular}




\begin{tabular}{|l|l|l|l|}
\hline 8 & -5.578995 & -1.082294 & -1.032472 \\
\hline 7 & -3.016766 & -0.371696 & -0.451464 \\
\hline 1 & -2.553281 & -0.150757 & -1.332803 \\
\hline 1 & -2.323692 & -0.779819 & 0.179602 \\
\hline 6 & -3.557031 & 0.871501 & 0.105735 \\
\hline 1 & -4.468978 & 1.118367 & -0.457692 \\
\hline 6 & -2.554660 & 1.993901 & -0.198660 \\
\hline 8 & -1.940940 & 1.977588 & -1.269727 \\
\hline 7 & -2.358302 & 2.966621 & 0.707012 \\
\hline 6 & -1.349783 & 3.991253 & 0.477421 \\
\hline 1 & -2.802446 & 2.921176 & 1.613356 \\
\hline 1 & -1.646470 & 4.579184 & -0.399010 \\
\hline 6 & -0.010453 & 3.299918 & 0.191240 \\
\hline 8 & 0.374404 & 2.347956 & 0.872856 \\
\hline 7 & 0.704148 & 3.782911 & -0.844068 \\
\hline 6 & 2.001278 & 3.222059 & -1.223073 \\
\hline 1 & 0.329481 & 4.554042 & -1.379760 \\
\hline 1 & 2.247417 & 3.653373 & -2.200214 \\
\hline 6 & 1.888994 & 1.705111 & -1.457803 \\
\hline 8 & 2.851102 & 0.961395 & -1.257671 \\
\hline 7 & 0.706443 & 1.255138 & -1.917715 \\
\hline 6 & 0.451308 & -0.147371 & -2.190609 \\
\hline 1 & -0.093053 & 1.880578 & -1.939277 \\
\hline 1 & -0.610144 & -0.198528 & -2.464919 \\
\hline 6 & 0.599140 & -1.044236 & -0.950078 \\
\hline 8 & 0.741477 & -2.267379 & -1.088877 \\
\hline 7 & 0.548156 & -0.470167 & 0.265499 \\
\hline 6 & 0.879447 & -1.222407 & 1.458063 \\
\hline 1 & 0.455159 & 0.544849 & 0.352156 \\
\hline 1 & 1.524730 & -2.057181 & 1.152752 \\
\hline 6 & -0.345242 & -1.914261 & 2.038739 \\
\hline 8 & -1.421167 & -2.018130 & 1.477187 \\
\hline 1 & 1.606339 & -0.347177 & 2.492615 \\
\hline 1 & 0.936178 & 0.464110 & 2.800694 \\
\hline 6 & 1.798823 & -0.961175 & 3.376813 \\
\hline 1 & 2.914702 & 0.275089 & 1.993596 \\
\hline 1 & 2.721487 & 0.974369 & 1.174164 \\
\hline 1 & 3.328537 & 0.869618 & 2.813623 \\
\hline 3.996318 & -0.715556 & 1.557698 \\
\hline 463588 & -0.200567 & 1.514786 \\
\hline 1 & & & \\
\hline 1 & & & \\
\hline 1.53873 & -538158 & 2.27929 \\
\hline 1 & & & \\
\hline 1 & & &
\end{tabular}




\begin{tabular}{|l|l|l|l|}
\hline 7 & 3.678243 & -1.285205 & 0.243346 \\
\hline 1 & 3.243556 & -0.648063 & -0.433393 \\
\hline 6 & 4.147813 & -2.442011 & -0.213074 \\
\hline 7 & 3.598861 & -2.983365 & -1.306491 \\
\hline 1 & 4.045635 & -3.755053 & -1.778714 \\
\hline 1 & 2.631053 & -2.762614 & -1.536316 \\
\hline 7 & 5.137314 & -3.076322 & 0.428115 \\
\hline 1 & 5.749563 & -2.578619 & 1.057780 \\
\hline 1 & 5.385820 & -4.025780 & 0.192145 \\
\hline 8 & -0.096800 & -2.454706 & 3.221841 \\
\hline 1 & -0.882897 & -2.926261 & 3.544205 \\
\hline 6 & -4.007099 & -3.907939 & 0.412465 \\
\hline 1 & -3.575190 & -3.272743 & 1.189456 \\
\hline 1 & -5.094749 & -3.908130 & 0.487177 \\
\hline 1 & -3.650694 & -4.933133 & 0.560196 \\
\hline 6 & -3.907488 & 0.682013 & 1.575152 \\
\hline 1 & -4.445573 & 1.545075 & 1.975474 \\
\hline 1 & -4.570423 & -0.179901 & 1.685756 \\
\hline 1 & -3.009736 & 0.502915 & 2.177114 \\
\hline 6 & -1.224232 & 4.887621 & 1.706073 \\
\hline 1 & -0.478270 & 5.666380 & 1.534104 \\
\hline 1 & -2.178478 & 5.376053 & 1.917895 \\
\hline 1 & -0.913751 & 4.299194 & 2.574042 \\
\hline 6 & 3.104747 & 3.566915 & -0.228734 \\
\hline 1 & 4.041478 & 3.100681 & -0.536889 \\
\hline 1 & 3.241218 & 4.649423 & -0.196488 \\
\hline 1 & 2.841490 & 3.214380 & 0.771034 \\
\hline 6 & 1.274485 & -0.686828 & -3.362274 \\
\hline 1 & 1.006636 & -1.724452 & -3.566492 \\
\hline 1 & 1.067601 & -0.087735 & -4.250725 \\
\hline 1 & 2.342942 & -0.625852 & -3.143949 \\
\hline & & & \\
\hline 1 & & & \\
\hline 1 & & & \\
\hline 1 & & &
\end{tabular}

$b_{2}-y_{4} T S$ 


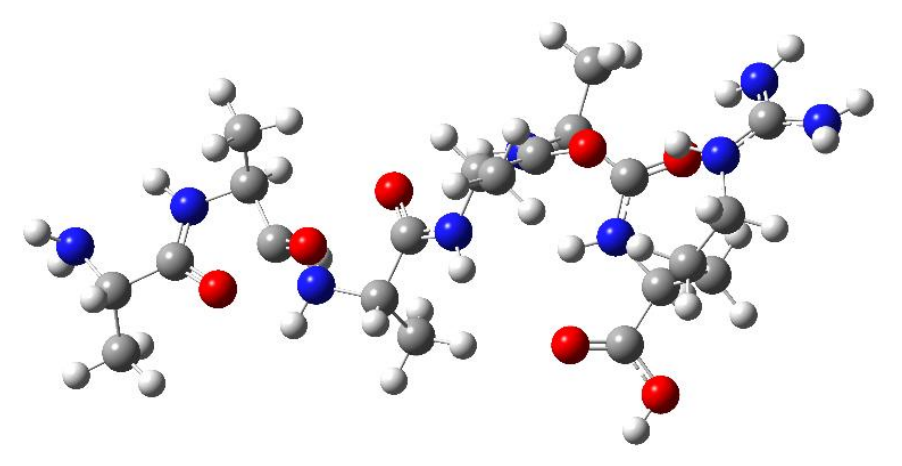

\begin{tabular}{|c|c|c|c|}
\hline $\begin{array}{c}\text { Atom } \\
\text { number }\end{array}$ & $\mathrm{x}$ & $\mathrm{y}$ & $\mathrm{z}$ \\
\hline 7 & 8.76491 & -0.524629 & -0.89857 \\
\hline 1 & 9.142703 & 0.000408 & -1.680311 \\
\hline 1 & 9.5233 & -1.049904 & -0.478476 \\
\hline 6 & 8.081046 & 0.333731 & 0.056149 \\
\hline 1 & 8.493823 & 0.217971 & 1.066602 \\
\hline 6 & 6.648918 & -0.136282 & 0.16118 \\
\hline 8 & 5.80279 & 0.412168 & 0.958455 \\
\hline 7 & 6.183874 & -1.126885 & -0.555622 \\
\hline 6 & 4.824076 & -1.509645 & -0.180952 \\
\hline 1 & 6.831909 & -1.629222 & -1.161894 \\
\hline 1 & 4.168328 & -1.52198 & -1.054546 \\
\hline 6 & 4.416616 & -0.36043 & 0.768341 \\
\hline 8 & 3.717289 & -0.440363 & 1.733908 \\
\hline 7 & 3.712955 & 0.961463 & -0.366434 \\
\hline 1 & 3.594814 & 0.606711 & -1.31889 \\
\hline 1 & 4.324383 & 1.777929 & -0.385118 \\
\hline 6 & 2.385461 & 1.3266 & 0.166806 \\
\hline 1 & 2.529715 & 1.597272 & 1.216589 \\
\hline 6 & 1.546848 & 0.053834 & 0.070128 \\
\hline 8 & 1.758017 & -0.746893 & -0.846084 \\
\hline 7 & 0.570495 & -0.107299 & 0.976384 \\
\hline 6 & -0.18236 & -1.35915 & 1.100719 \\
\hline 1 & 0.49465 & 0.55771 & 1.7363 \\
\hline 1 & 0.477953 & -2.158136 & 0.742002 \\
\hline 6 & -1.418303 & -1.332327 & 0.187121 \\
\hline 8 & -2.558212 & -1.434263 & 0.635588 \\
\hline 7 & -1.166082 & -1.149593 & -1.127642 \\
\hline 6 & -2.248912 & -1.138166 & -2.107076 \\
\hline 1 & -0.198368 & -1.179477 & -1.438194 \\
\hline 1 & -1.80762 & -0.792492 & -3.049127 \\
\hline & & & \\
\hline 1 & & & \\
\hline 1 & & & \\
\hline & & & \\
\hline 1 & & &
\end{tabular}




\begin{tabular}{|c|c|c|c|}
\hline 6 & -3.309504 & -0.084665 & -1.737055 \\
\hline 8 & -4.499827 & -0.278506 & -1.979256 \\
\hline 7 & -2.839678 & 1.054628 & -1.196856 \\
\hline 6 & -3.654868 & 2.198369 & -0.824267 \\
\hline 1 & -1.851489 & 1.122011 & -0.978796 \\
\hline 1 & -4.07748 & 2.668269 & -1.721161 \\
\hline 6 & -2.713052 & 3.198118 & -0.172629 \\
\hline 8 & -1.550998 & 2.969007 & 0.089476 \\
\hline 6 & -4.815359 & 1.865384 & 0.135815 \\
\hline 1 & -5.3623 & 2.792778 & 0.326619 \\
\hline 1 & -5.482489 & 1.198009 & -0.413369 \\
\hline 6 & -4.356972 & 1.216659 & 1.44967 \\
\hline 1 & -3.379439 & 0.737775 & 1.317855 \\
\hline 1 & -4.2436 & 1.966769 & 2.239119 \\
\hline 6 & -5.336155 & 0.144395 & 1.935767 \\
\hline 1 & -5.020634 & -0.259474 & 2.902335 \\
\hline 1 & -6.337733 & 0.57149 & 2.045732 \\
\hline 7 & -5.395814 & -0.930759 & 0.942785 \\
\hline 1 & -4.501442 & -1.208985 & 0.535776 \\
\hline 6 & -6.49621 & -1.427157 & 0.390236 \\
\hline 7 & -7.672847 & -1.35056 & 1.02915 \\
\hline 1 & -8.535888 & -1.565285 & 0.552962 \\
\hline 1 & -7.710752 & -1.214075 & 2.027892 \\
\hline 7 & -6.417313 & -2.009027 & -0.810051 \\
\hline 1 & -7.189227 & -2.549611 & -1.169895 \\
\hline 1 & -5.698568 & -1.678382 & -1.455633 \\
\hline 8 & -3.325832 & 4.343163 & 0.102088 \\
\hline 1 & -2.699978 & 4.955353 & 0.522542 \\
\hline 6 & 8.105734 & 1.819897 & -0.316726 \\
\hline 1 & 7.53153 & 2.41379 & 0.39721 \\
\hline 1 & 9.138142 & 2.174149 & -0.307879 \\
\hline 1 & 7.695869 & 1.969811 & -1.32123 \\
\hline 1 & 4.774992 & -2.844174 & 0.551274 \\
\hline 1 & 3.745671 & -3.039952 & 0.857765 \\
\hline 1 & 5.10606 & -3.649375 & -0.108083 \\
\hline 1 & 5.404116 & -2.824244 & 1.444218 \\
\hline 1 & 1.738413 & 2.46537 & -0.6211 \\
\hline & 1.606182 & 2.179518 & -1.669448 \\
\hline 0.757916 & 2.709537 & -0.204285 \\
\hline 2.360004 & 3.363714 & -0.57695 \\
\hline-0.566564 & -1.592642 & 2.551575 \\
\hline & & & \\
\hline 1 & & & \\
\hline 1 & & &
\end{tabular}




\begin{tabular}{|l|l|l|l|}
\hline 1 & -1.211218 & -0.787486 & 2.916621 \\
\hline 1 & -1.122365 & -2.526202 & 2.645685 \\
\hline 1 & 0.331983 & -1.649352 & 3.169631 \\
\hline 6 & -2.872699 & -2.513419 & -2.321688 \\
\hline 1 & -3.645142 & -2.456723 & -3.090316 \\
\hline 1 & -2.104707 & -3.216235 & -2.650743 \\
\hline 1 & -3.314789 & -2.886083 & -1.394231 \\
\hline
\end{tabular}

GGR

Minimum

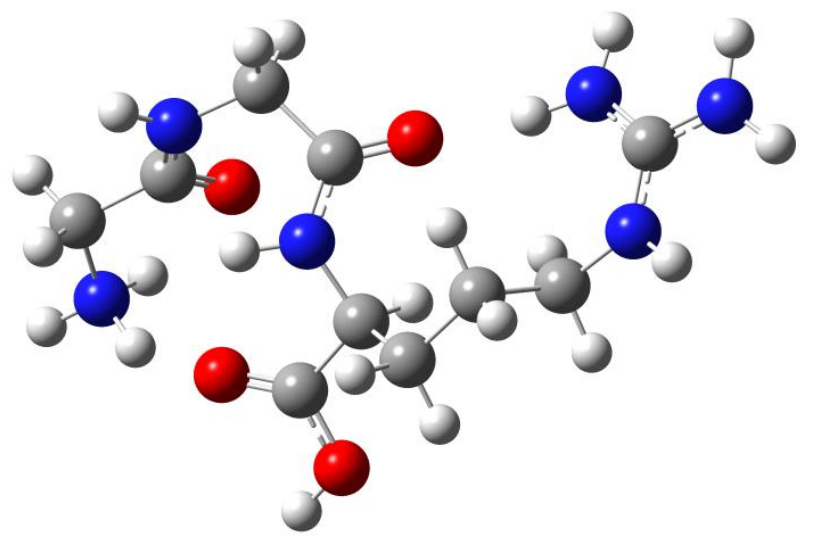

\begin{tabular}{|c|c|c|c|}
\hline $\begin{array}{c}\text { Atom } \\
\text { number }\end{array}$ & $\mathrm{x}$ & $\mathrm{y}$ & $\mathrm{z}$ \\
\hline 7 & 5.1 & 1.814081 & -0.717531 \\
\hline 6 & 5.22476 & 0.752828 & -1.485828 \\
\hline 6 & 4.943928 & -0.944409 & -0.514643 \\
\hline 8 & 4.663124 & -2.042288 & -0.501529 \\
\hline 7 & 4.339565 & -0.186771 & 1.59746 \\
\hline 6 & 3.023841 & -0.791296 & 1.761315 \\
\hline 6 & 2.065448 & -0.255241 & 0.705248 \\
\hline 8 & 2.457156 & 0.463155 & -0.226421 \\
\hline 7 & 0.776377 & -0.614565 & 0.83726 \\
\hline 6 & -0.246265 & -0.341007 & -0.178728 \\
\hline 6 & -0.711263 & 1.12831 & -0.066818 \\
\hline 8 & -1.850002 & 1.432671 & 0.210576 \\
\hline 8 & 0.210642 & 2.045909 & -0.289833 \\
\hline 6 & -1.423993 & -1.300997 & -0.027504 \\
\hline 6 & -2.388185 & -1.234123 & -1.231501 \\
\hline 6 & -3.861225 & -1.319182 & -0.835089 \\
\hline 7 & -4.23031 & -0.103591 & -0.106316 \\
\hline
\end{tabular}




\begin{tabular}{|c|c|c|c|}
\hline 6 & -5.47335 & 0.259297 & 0.184607 \\
\hline 7 & -5.707078 & 1.476657 & 0.686731 \\
\hline 7 & -6.493596 & -0.577881 & -0.024429 \\
\hline 1 & 5.900449 & 2.243323 & -0.273344 \\
\hline 1 & 4.178023 & 2.163036 & -0.490552 \\
\hline 1 & 6.218574 & 0.505343 & -1.847732 \\
\hline 1 & 4.384127 & 0.510842 & -2.130252 \\
\hline 1 & 5.000631 & -0.607896 & 2.246966 \\
\hline 1 & 4.31236 & 0.809099 & 1.798084 \\
\hline 1 & 3.115453 & -1.874688 & 1.616592 \\
\hline 1 & 2.588451 & -0.632339 & 2.756712 \\
\hline 1 & 0.497759 & -1.153938 & 1.648805 \\
\hline 1 & 0.229398 & -0.469993 & -1.160275 \\
\hline 1 & 1.075269 & 1.635499 & -0.496259 \\
\hline 1 & -1.035207 & -2.31842 & 0.077168 \\
\hline 1 & -1.949474 & -1.054635 & 0.903664 \\
\hline 1 & -2.175046 & -2.052509 & -1.923437 \\
\hline 1 & -2.24626 & -0.304907 & -1.795552 \\
\hline 1 & -4.478322 & -1.401096 & -1.736565 \\
\hline 1 & -4.034552 & -2.203254 & -0.207028 \\
\hline 1 & -3.47021 & 0.541243 & 0.120223 \\
\hline 1 & -6.616936 & 1.741442 & 1.033402 \\
\hline 1 & -4.968724 & 2.164153 & 0.738929 \\
\hline 1 & -7.450413 & -0.280079 & 0.097023 \\
\hline 1 & -6.339594 & -1.536378 & -0.297507 \\
\hline
\end{tabular}

$a_{1}-y_{2}$ TS

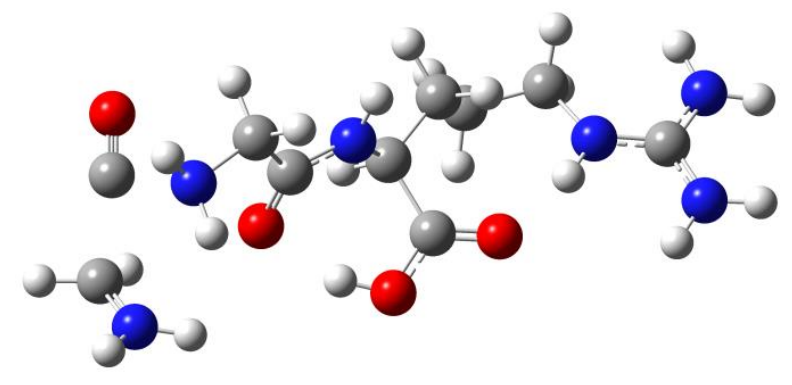

\begin{tabular}{|c|c|c|c|}
\hline $\begin{array}{c}\text { Atom } \\
\text { number }\end{array}$ & $\mathrm{x}$ & $\mathrm{y}$ & $\mathrm{z}$ \\
\hline 7 & 5.1 & 1.814081 & -0.717531 \\
\hline 6 & 5.22476 & 0.752828 & -1.485828 \\
\hline 6 & 4.943928 & -0.944409 & -0.514643 \\
\hline 8 & 4.663124 & -2.042288 & -0.501529 \\
\hline
\end{tabular}




\begin{tabular}{|c|c|c|c|}
\hline 7 & 4.339565 & -0.186771 & 1.59746 \\
\hline 6 & 3.023841 & -0.791296 & 1.761315 \\
\hline 6 & 2.065448 & -0.255241 & 0.705248 \\
\hline 8 & 2.457156 & 0.463155 & -0.226421 \\
\hline 7 & 0.776377 & -0.614565 & 0.83726 \\
\hline 6 & -0.246265 & -0.341007 & -0.178728 \\
\hline 6 & -0.711263 & 1.12831 & -0.066818 \\
\hline 8 & -1.850002 & 1.432671 & 0.210576 \\
\hline 8 & 0.210642 & 2.045909 & -0.289833 \\
\hline 6 & -1.423993 & -1.300997 & -0.027504 \\
\hline 6 & -2.388185 & -1.234123 & -1.231501 \\
\hline 6 & -3.861225 & -1.319182 & -0.835089 \\
\hline 7 & -4.23031 & -0.103591 & -0.106316 \\
\hline 6 & -5.47335 & 0.259297 & 0.184607 \\
\hline 7 & -5.707078 & 1.476657 & 0.686731 \\
\hline 7 & -6.493596 & -0.577881 & -0.024429 \\
\hline 1 & 5.900449 & 2.243323 & -0.273344 \\
\hline 1 & 4.178023 & 2.163036 & -0.490552 \\
\hline 1 & 6.218574 & 0.505343 & -1.847732 \\
\hline 1 & 4.384127 & 0.510842 & -2.130252 \\
\hline 1 & 5.000631 & -0.607896 & 2.246966 \\
\hline 1 & 4.31236 & 0.809099 & 1.798084 \\
\hline 1 & 3.115453 & -1.874688 & 1.616592 \\
\hline 1 & 2.588451 & -0.632339 & 2.756712 \\
\hline 1 & 0.497759 & -1.153938 & 1.648805 \\
\hline 1 & 0.229398 & -0.469993 & -1.160275 \\
\hline 1 & 1.075269 & 1.635499 & -0.496259 \\
\hline 1 & -1.035207 & -2.31842 & 0.077168 \\
\hline 1 & -1.949474 & -1.054635 & 0.903664 \\
\hline 1 & -2.175046 & -2.052509 & -1.923437 \\
\hline 1 & -2.24626 & -0.304907 & -1.795552 \\
\hline 1 & -4.478322 & -1.401096 & -1.736565 \\
\hline 1 & -4.034552 & -2.203254 & -0.207028 \\
\hline 1 & -3.47021 & 0.541243 & 0.120223 \\
\hline 1 & -6.616936 & 1.741442 & 1.033402 \\
\hline 1 & -4.968724 & 2.164153 & 0.738929 \\
\hline 1 & -7.450413 & -0.280079 & 0.097023 \\
\hline 1 & -6.339594 & -1.536378 & -0.297507 \\
\hline & & & \\
\hline 1 & & & \\
\hline & & & \\
\hline & & &
\end{tabular}

$b_{2}-y_{1}$ TS 


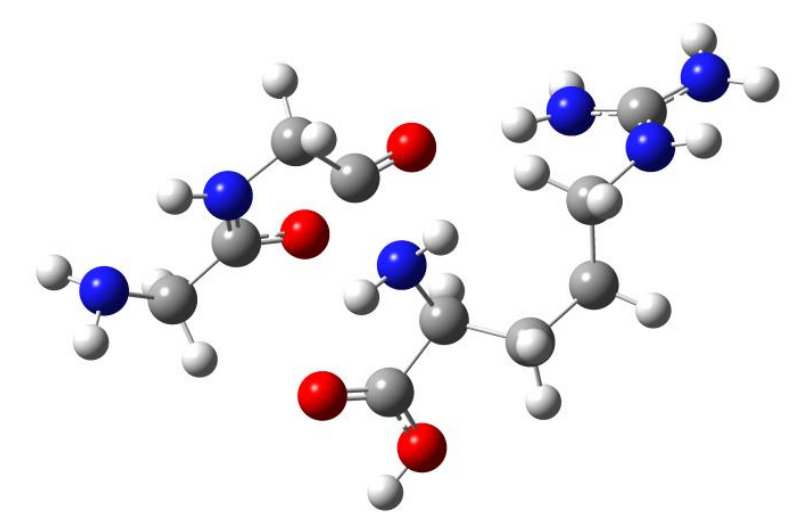

\begin{tabular}{|c|c|c|c|}
\hline $\begin{array}{c}\text { Atom } \\
\text { number }\end{array}$ & $\mathrm{x}$ & $\mathrm{y}$ & $\mathrm{z}$ \\
\hline 7 & 5.254171 & -0.930087 & -0.170321 \\
\hline 1 & 5.793795 & -0.14905 & 0.18487 \\
\hline 1 & 5.88077 & -1.543306 & -0.679179 \\
\hline 6 & 4.121336 & -0.502754 & -0.965008 \\
\hline 1 & 4.018688 & 0.586391 & -1.032359 \\
\hline 6 & 2.869187 & -1.003245 & -0.311414 \\
\hline 8 & 1.678327 & -0.814328 & -0.810884 \\
\hline 7 & 2.876841 & -1.679275 & 0.800562 \\
\hline 6 & 1.542283 & -2.139563 & 1.154417 \\
\hline 1 & 3.778193 & -1.905948 & 1.227005 \\
\hline 1 & 1.427048 & -3.20762 & 0.942063 \\
\hline 6 & 0.686832 & -1.338806 & 0.168237 \\
\hline 8 & -0.428584 & -1.562708 & -0.222731 \\
\hline 7 & 0.552296 & 0.395852 & 1.145011 \\
\hline 6 & 0.069999 & 1.399034 & 0.174515 \\
\hline 1 & 1.422399 & 0.732582 & 1.568826 \\
\hline 1 & -0.24652 & 0.85745 & -0.731523 \\
\hline 6 & 1.291929 & 2.207474 & -0.252188 \\
\hline 8 & 2.41546 & 1.942813 & 0.118348 \\
\hline 6 & -1.114925 & 2.261283 & 0.652914 \\
\hline 1 & -1.018438 & 2.456261 & 1.728741 \\
\hline 1 & -1.049577 & 3.232532 & 0.156749 \\
\hline 6 & -2.48575 & 1.660951 & 0.313781 \\
\hline 1 & -3.258135 & 2.40624 & 0.528042 \\
\hline 1 & -2.530282 & 1.461905 & -0.764317 \\
\hline 6 & -2.821849 & 0.378563 & 1.078787 \\
\hline 1 & -2.052717 & -0.389884 & 0.950804 \\
\hline 1 & -2.899279 & 0.576051 & 2.151809 \\
\hline & & & \\
\hline
\end{tabular}




\begin{tabular}{|c|c|c|c|}
\hline 7 & -4.109583 & -0.188674 & 0.669879 \\
\hline 1 & -4.92478 & 0.053862 & 1.217228 \\
\hline 6 & -4.308811 & -0.908629 & -0.438032 \\
\hline 7 & -3.27643 & -1.372348 & -1.142109 \\
\hline 1 & -2.328644 & -1.393523 & -0.778196 \\
\hline 1 & -3.436993 & -1.84668 & -2.019041 \\
\hline 7 & -5.55568 & -1.157452 & -0.848154 \\
\hline 1 & -6.352386 & -0.698026 & -0.43114 \\
\hline 1 & -5.746562 & -1.84159 & -1.566104 \\
\hline 8 & 0.979651 & 3.180516 & -1.089162 \\
\hline 1 & 1.778215 & 3.669394 & -1.353222 \\
\hline 1 & -0.126401 & 0.239545 & 1.888753 \\
\hline 1 & 1.294662 & -1.939416 & 2.196724 \\
\hline 1 & 4.12542 & -0.889479 & -1.99122 \\
\hline
\end{tabular}

$\mathrm{G}_{3} \mathrm{R}$

Minimum

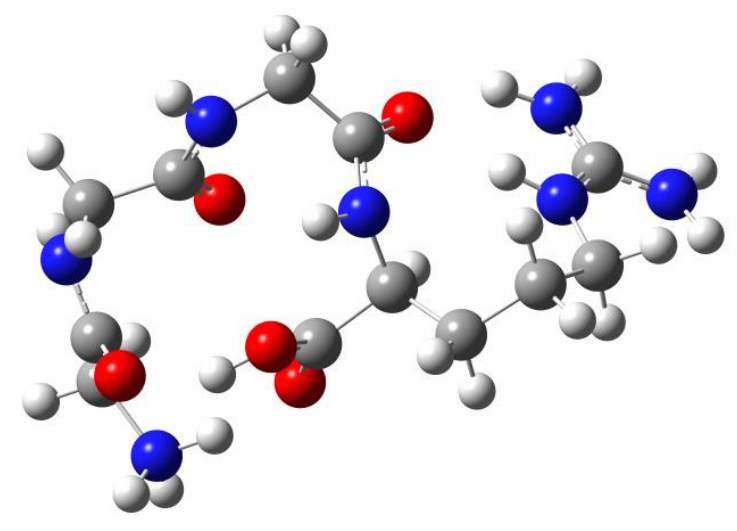

\begin{tabular}{|c|c|c|c|}
\hline $\begin{array}{c}\text { Atom } \\
\text { number }\end{array}$ & $\mathrm{x}$ & $\mathrm{y}$ & $\mathrm{z}$ \\
\hline 7 & 3.474685 & -2.77823 & 1.162071 \\
\hline 1 & 3.446293 & -3.50679 & 1.879985 \\
\hline 1 & 2.50997 & -2.61387 & 0.773229 \\
\hline 1 & 4.052406 & -3.09892 & 0.377052 \\
\hline 6 & 4.00652 & -1.48994 & 1.694187 \\
\hline 1 & 3.349127 & -1.15773 & 2.500592 \\
\hline 6 & 3.974062 & -0.50923 & 0.523048 \\
\hline 8 & 3.804412 & -0.96135 & -0.60808 \\
\hline 7 & 4.120723 & 0.792579 & 0.80167 \\
\hline 6 & 3.599246 & 1.774208 & -0.12982 \\
\hline
\end{tabular}




\begin{tabular}{|c|c|c|c|}
\hline 1 & 4.145863 & 1.067103 & 1.776347 \\
\hline 1 & 4.050392 & 2.747911 & 0.075712 \\
\hline 1 & 3.863399 & 1.48051 & -1.14914 \\
\hline 6 & 2.069525 & 1.833102 & 0.03996 \\
\hline 8 & 1.501061 & 1.093388 & 0.831547 \\
\hline 7 & 1.389636 & 2.716409 & -0.73558 \\
\hline 6 & -0.0096 & 3.009105 & -0.43808 \\
\hline 1 & 1.901284 & 3.367419 & -1.3174 \\
\hline 1 & -0.1155 & 3.535908 & 0.514446 \\
\hline 1 & -0.41017 & 3.642156 & -1.23448 \\
\hline 6 & -0.85504 & 1.746315 & -0.3119 \\
\hline 8 & -1.73021 & 1.670396 & 0.548483 \\
\hline 7 & -0.61131 & 0.748628 & -1.19631 \\
\hline 6 & -0.76759 & -0.65177 & -0.80023 \\
\hline 1 & 0.132402 & 0.91806 & -1.86567 \\
\hline 1 & -1.18787 & -0.67404 & 0.208103 \\
\hline 6 & 0.631757 & -1.25251 & -0.70118 \\
\hline 8 & 0.995963 & -1.99484 & 0.195361 \\
\hline 6 & -1.61461 & -1.47215 & -1.79058 \\
\hline 1 & -1.08742 & -1.48662 & -2.75111 \\
\hline 1 & -1.65146 & -2.50811 & -1.43248 \\
\hline 6 & -3.03535 & -0.9501 & -2.03744 \\
\hline 1 & -3.00553 & 0.110068 & -2.31776 \\
\hline 1 & -3.43083 & -1.48175 & -2.90725 \\
\hline 6 & -4.04379 & -1.14566 & -0.90382 \\
\hline 1 & -5.05286 & -0.98937 & -1.30429 \\
\hline 1 & -3.9798 & -2.16926 & -0.51146 \\
\hline 7 & -3.79837 & -0.19088 & 0.175372 \\
\hline 1 & -3.04515 & 0.494706 & 0.081871 \\
\hline 6 & -4.51645 & -0.07937 & 1.286109 \\
\hline 7 & -5.57537 & -0.86679 & 1.499528 \\
\hline 1 & -6.11794 & -0.79877 & 2.347674 \\
\hline 1 & -5.90983 & -1.49711 & 0.786867 \\
\hline 7 & -4.14872 & 0.821338 & 2.204062 \\
\hline 1 & -3.33536 & 1.402605 & 2.034323 \\
\hline 1 & -4.72184 & 1.019052 & 3.009821 \\
\hline 8 & 1.386915 & -0.88333 & -1.7215 \\
\hline 1 & 2.324804 & -1.12998 & -1.57423 \\
\hline 1 & 5.019462 & -1.64076 & 2.075536 \\
\hline & & & \\
\hline 1 & & & \\
\hline 1 & & &
\end{tabular}

$a_{1}-y_{3}$ TS 


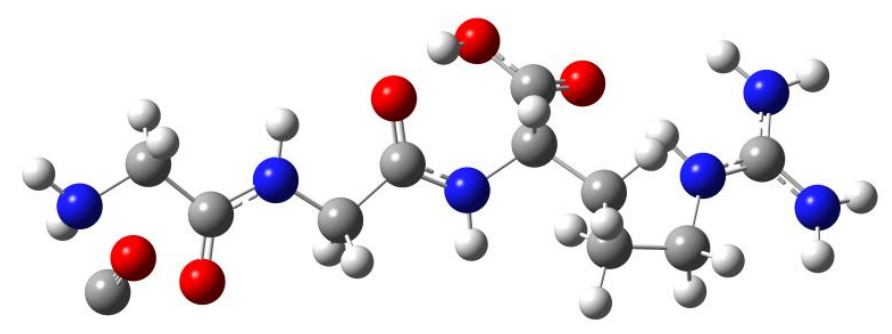

.

\begin{tabular}{|c|c|c|c|}
\hline $\begin{array}{l}\text { Atom } \\
\text { number }\end{array}$ & $\mathrm{x}$ & $y$ & z \\
\hline 7 & 6.621162 & 2.046962 & -1.469083 \\
\hline 6 & 6.763021 & 2.254627 & -0.179967 \\
\hline 6 & 6.781703 & 0.556256 & 0.877621 \\
\hline 8 & 6.600961 & 0.012886 & 1.857156 \\
\hline 7 & 6.295211 & -1.005394 & -0.739028 \\
\hline 6 & 5.008691 & -1.525751 & -0.288415 \\
\hline 6 & 3.960014 & -0.415744 & -0.310941 \\
\hline 8 & 4.246221 & 0.752092 & -0.582484 \\
\hline 7 & 2.710067 & -0.801658 & -0.009978 \\
\hline 6 & 1.610449 & 0.134618 & 0.028889 \\
\hline 6 & 0.344194 & -0.644319 & 0.353700 \\
\hline 8 & 0.393835 & -1.847357 & 0.610932 \\
\hline 7 & -0.820270 & 0.037355 & 0.353182 \\
\hline 6 & -2.058712 & -0.551850 & 0.876500 \\
\hline 6 & -2.695115 & -1.531515 & -0.135677 \\
\hline 8 & -3.841131 & -1.412389 & -0.522448 \\
\hline 8 & -1.947817 & -2.532265 & -0.539615 \\
\hline 6 & -3.043005 & 0.529448 & 1.317103 \\
\hline 6 & -3.456579 & 1.529572 & 0.212621 \\
\hline 6 & -4.954794 & 1.840240 & 0.229505 \\
\hline 7 & -5.683007 & 0.626549 & -0.136224 \\
\hline 6 & -6.989444 & 0.546626 & -0.341109 \\
\hline 7 & -7.514761 & -0.594380 & -0.801721 \\
\hline 7 & -7.786117 & 1.593478 & -0.100301 \\
\hline 1 & 7.419195 & 1.976224 & -2.085165 \\
\hline 1 & 5.699076 & 1.813636 & -1.820817 \\
\hline 1 & 5.871649 & 2.548533 & 0.366270 \\
\hline 1 & 7.721867 & 2.617453 & 0.177149 \\
\hline 1 & 6.250754 & -0.719413 & -1.712861 \\
\hline 1 & 7.010910 & -1.725007 & -0.664826 \\
\hline 1 & 5.116566 & -1.879043 & 0.744006 \\
\hline
\end{tabular}




\begin{tabular}{|c|c|c|c|}
\hline 1 & 4.650328 & -2.373461 & -0.886140 \\
\hline 1 & 2.487079 & -1.764214 & 0.226514 \\
\hline 1 & 1.505328 & 0.646393 & -0.935229 \\
\hline 1 & 1.776535 & 0.902223 & 0.794526 \\
\hline 1 & -0.823222 & 1.016717 & 0.097916 \\
\hline 1 & -1.777144 & -1.157496 & 1.748119 \\
\hline 1 & -1.069603 & -2.539512 & -0.096174 \\
\hline 1 & -2.606874 & 1.063505 & 2.166670 \\
\hline 1 & -3.923686 & 0.007416 & 1.705185 \\
\hline 1 & -2.926027 & 2.478619 & 0.333616 \\
\hline 1 & -3.206638 & 1.145535 & -0.784164 \\
\hline 1 & -5.255518 & 2.174381 & 1.231668 \\
\hline 1 & -5.177840 & 2.635969 & -0.490380 \\
\hline 1 & -5.108880 & -0.209539 & -0.296747 \\
\hline 1 & -8.511789 & -0.731323 & -0.869860 \\
\hline 1 & -6.917551 & -1.371180 & -1.049075 \\
\hline 1 & -7.429365 & 2.439233 & 0.316568 \\
\hline 1 & -8.770213 & 1.566995 & -0.321992 \\
\hline
\end{tabular}

$b_{2}-y_{2}$ TS

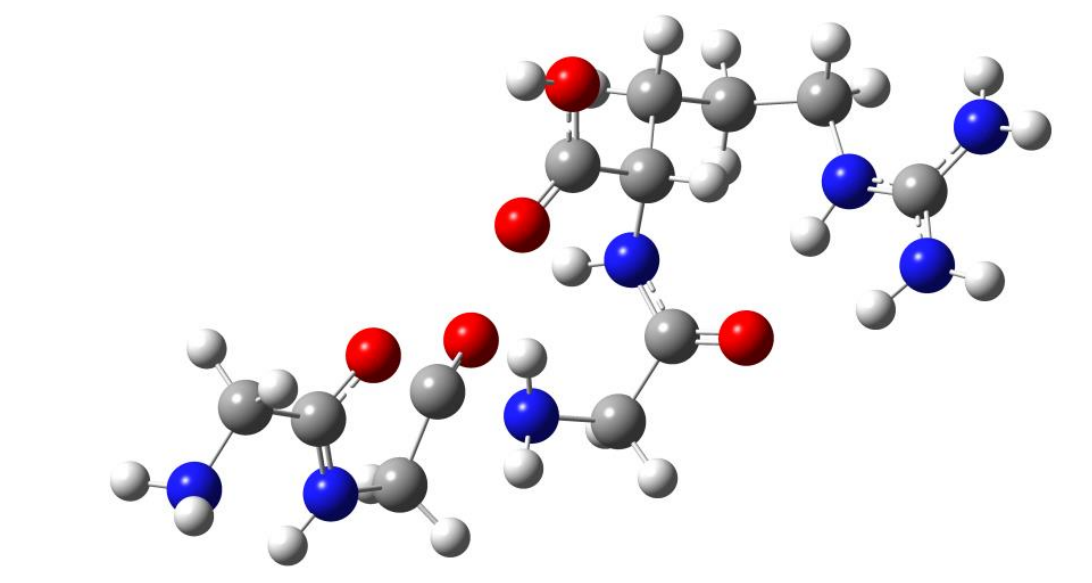

\begin{tabular}{|c|c|c|c|}
\hline $\begin{array}{c}\text { Atom } \\
\text { number }\end{array}$ & $\mathrm{x}$ & $\mathrm{y}$ & $\mathrm{z}$ \\
\hline 7 & 6.739330 & 0.320828 & -1.031258 \\
\hline 1 & 6.981901 & 0.195842 & -2.006951 \\
\hline 1 & 7.516035 & 0.779700 & -0.568866 \\
\hline 6 & 5.487647 & 1.025262 & -0.850285 \\
\hline 1 & 5.580777 & 1.968773 & -0.299110 \\
\hline 6 & 4.550186 & 0.156042 & -0.062699 \\
\hline 8 & 3.365508 & 0.545313 & 0.284290 \\
\hline 7 & 4.870437 & -1.045567 & 0.331750 \\
\hline
\end{tabular}




\begin{tabular}{|l|l|l|l|}
\hline 6 & 3.838851 & -1.624474 & 1.179708 \\
\hline 1 & 5.807966 & -1.395878 & 0.128917 \\
\hline 1 & 3.538104 & -2.619852 & 0.850240 \\
\hline 6 & 2.709457 & -0.593959 & 1.047126 \\
\hline 8 & 1.867180 & -0.327824 & 1.857148 \\
\hline 7 & 1.708729 & -1.210979 & -0.528373 \\
\hline 6 & 0.559569 & -2.025748 & -0.055377 \\
\hline 1 & 2.214109 & -1.693434 & -1.270942 \\
\hline 1 & 0.450576 & -2.935641 & -0.644442 \\
\hline 6 & -0.731598 & -1.225137 & -0.173246 \\
\hline 8 & -1.668695 & -1.605736 & -0.871491 \\
\hline 7 & -0.747535 & -0.061125 & 0.517755 \\
\hline 6 & -1.497748 & 1.091063 & 0.048407 \\
\hline 1 & 0.065644 & 0.135551 & 1.102416 \\
\hline 1 & -2.236274 & 0.732525 & -0.677190 \\
\hline 6 & -0.530887 & 1.961257 & -0.761624 \\
\hline 8 & 0.570529 & 1.591620 & -1.109560 \\
\hline 6 & -2.198445 & 1.848301 & 1.184730 \\
\hline 1 & -1.435457 & 2.241919 & 1.866400 \\
\hline 1 & -2.708111 & 2.715574 & 0.754277 \\
\hline 6 & -3.184541 & 1.002574 & 1.999411 \\
\hline 1 & -2.684373 & 0.117304 & 2.411525 \\
\hline 1 & -3.499670 & 1.597496 & 2.860914 \\
\hline 6 & -4.457968 & 0.570026 & 1.270925 \\
\hline 1 & -5.187725 & 0.214243 & 2.007435 \\
\hline 1 & -4.891458 & 1.422095 & 0.732467 \\
\hline 7 & -4.175033 & -0.498738 & 0.306627 \\
\hline 1 & -3.280715 & -0.977452 & 0.348821 \\
\hline 6 & -4.995208 & -0.901969 & -0.661268 \\
\hline 7 & -6.252468 & -0.451882 & -0.711196 \\
\hline 1 & -6.853501 & -0.669158 & -1.492724 \\
\hline 1 & -6.669241 & 0.026071 & 0.073647 \\
\hline 7 & -4.539053 & -1.737492 & -1.597189 \\
\hline 1 & -3.549888 & -1.962945 & -1.627885 \\
\hline 1 & -5.166699 & -2.208518 & -2.231105 \\
\hline 8 & -1.053860 & 3.139280 & -1.066275 \\
\hline 1 & -0.429480 & 3.648571 & -1.610426 \\
\hline 1 & 1.395770 & -0.303568 & -0.901375 \\
\hline 1 & 4.985026 & 1.271305 & -1.792897 \\
\hline 1 & 4.161084 & -1.658699 & 2.224643 \\
\hline 1 & 0.723560 & -2.287789 & 0.997653 \\
\hline & & & \\
\hline 1 & & & \\
\hline 1 & &
\end{tabular}


$\mathrm{G}_{4} \mathrm{R}$

Minimum

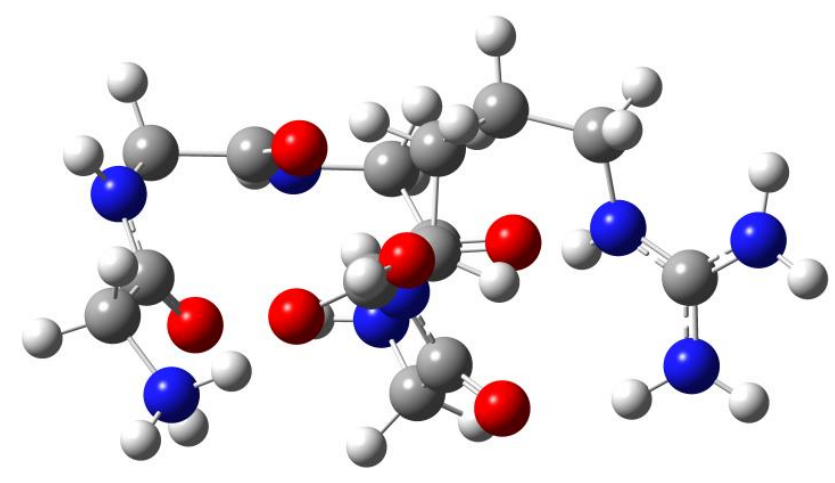

\begin{tabular}{|c|c|c|c|}
\hline $\begin{array}{c}\text { Atom } \\
\text { number }\end{array}$ & $\mathrm{x}$ & $\mathrm{y}$ & $\mathrm{z}$ \\
\hline 7 & -2.82971 & 2.560261 & 1.40906 \\
\hline 1 & -1.87006 & 2.676015 & 0.985984 \\
\hline 1 & -3.10293 & 3.444927 & 1.84319 \\
\hline 1 & -2.79412 & 1.813889 & 2.117279 \\
\hline 6 & -3.77482 & 2.137382 & 0.341224 \\
\hline 1 & -4.79681 & 2.388865 & 0.635496 \\
\hline 6 & -3.62894 & 0.620577 & 0.212718 \\
\hline 8 & -3.08528 & -0.00615 & 1.121414 \\
\hline 7 & -4.13243 & 0.050805 & -0.88729 \\
\hline 6 & -3.98624 & -1.37905 & -1.08803 \\
\hline 1 & -4.49263 & 0.620988 & -1.6402 \\
\hline 1 & -4.58632 & -1.92507 & -0.35337 \\
\hline 6 & -2.50855 & -1.76296 & -0.9809 \\
\hline 8 & -1.6202 & -0.99315 & -1.334 \\
\hline 7 & -2.24275 & -2.97031 & -0.44073 \\
\hline 6 & -0.86536 & -3.41015 & -0.30074 \\
\hline 1 & -2.99649 & -3.59447 & -0.18692 \\
\hline 1 & -0.85379 & -4.42171 & 0.111103 \\
\hline 1 & -0.35961 & -3.42731 & -1.27002 \\
\hline 6 & -0.04314 & -2.49978 & 0.606962 \\
\hline 8 & 1.17963 & -2.45009 & 0.491985 \\
\hline 7 & -0.70751 & -1.75661 & 1.514716 \\
\hline 6 & 0.026239 & -0.82888 & 2.342162 \\
\hline 1 & -1.719 & -1.73282 & 1.520375 \\
\hline & & & \\
\hline
\end{tabular}




\begin{tabular}{|c|c|c|c|}
\hline 1 & -0.66569 & -0.36092 & 3.046636 \\
\hline 1 & 0.794871 & -1.3474 & 2.921083 \\
\hline 6 & 0.746344 & 0.266477 & 1.557626 \\
\hline 8 & 1.734855 & 0.812541 & 2.038391 \\
\hline 7 & 0.242024 & 0.601156 & 0.343584 \\
\hline 6 & 0.971803 & 1.487623 & -0.53469 \\
\hline 1 & -0.49831 & 0.030525 & -0.06387 \\
\hline 1 & 1.990863 & 1.583179 & -0.14522 \\
\hline 6 & 0.382853 & 2.888456 & -0.51989 \\
\hline 8 & -0.69954 & 3.204846 & -0.04749 \\
\hline 6 & 0.996192 & 0.968191 & -1.98821 \\
\hline 1 & -0.0348 & 0.92327 & -2.35965 \\
\hline 1 & 1.531056 & 1.704243 & -2.59691 \\
\hline 6 & 1.624981 & -0.41659 & -2.1724 \\
\hline 1 & 1.049448 & -1.17414 & -1.63047 \\
\hline 1 & 1.539134 & -0.67002 & -3.23325 \\
\hline 6 & 3.102443 & -0.53494 & -1.79391 \\
\hline 1 & 3.526091 & -1.42751 & -2.27026 \\
\hline 1 & 3.655796 & 0.342411 & -2.1498 \\
\hline 7 & 3.265427 & -0.62167 & -0.34004 \\
\hline 1 & 2.555281 & -1.15338 & 0.169202 \\
\hline 6 & 4.392329 & -0.33297 & 0.310344 \\
\hline 7 & 4.372953 & -0.24448 & 1.641628 \\
\hline 1 & 3.495415 & -0.01688 & 2.106088 \\
\hline 1 & 5.231569 & -0.18913 & 2.168671 \\
\hline 7 & 5.525472 & -0.10512 & -0.36565 \\
\hline 1 & 5.647284 & -0.44704 & -1.3073 \\
\hline 1 & 6.340881 & 0.263974 & 0.101188 \\
\hline 8 & 1.170437 & 3.756691 & -1.12235 \\
\hline 1 & 0.77113 & 4.643498 & -1.11554 \\
\hline 1 & -3.51801 & 2.663488 & -0.58041 \\
\hline 1 & -4.35065 & -1.6276 & -2.08731 \\
\hline & & & \\
\hline 1 & & & \\
\hline 1 & & & \\
\hline & & &
\end{tabular}

$a_{1}-y_{4}$ TS 


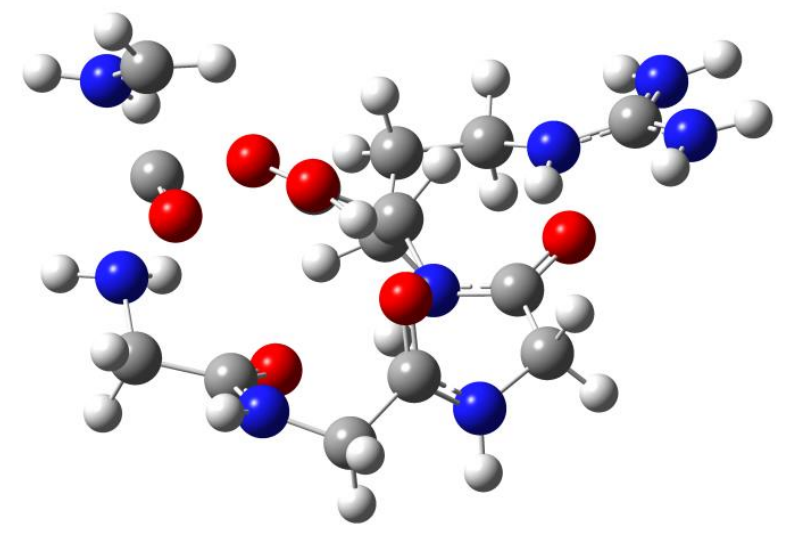

\begin{tabular}{|c|c|c|c|}
\hline $\begin{array}{c}\text { Atom } \\
\text { number }\end{array}$ & $\mathrm{x}$ & $\mathrm{y}$ & $\mathrm{z}$ \\
\hline 7 & -2.82971 & 2.560261 & 1.40906 \\
\hline 1 & -1.87006 & 2.676015 & 0.985984 \\
\hline 1 & -3.10293 & 3.444927 & 1.84319 \\
\hline 1 & -2.79412 & 1.813889 & 2.117279 \\
\hline 6 & -3.77482 & 2.137382 & 0.341224 \\
\hline 1 & -4.79681 & 2.388865 & 0.635496 \\
\hline 6 & -3.62894 & 0.620577 & 0.212718 \\
\hline 8 & -3.08528 & -0.00615 & 1.121414 \\
\hline 7 & -4.13243 & 0.050805 & -0.88729 \\
\hline 6 & -3.98624 & -1.37905 & -1.08803 \\
\hline 1 & -4.49263 & 0.620988 & -1.6402 \\
\hline 1 & -4.58632 & -1.92507 & -0.35337 \\
\hline 6 & -2.50855 & -1.76296 & -0.9809 \\
\hline 8 & -1.6202 & -0.99315 & -1.334 \\
\hline 7 & -2.24275 & -2.97031 & -0.44073 \\
\hline 6 & -0.86536 & -3.41015 & -0.30074 \\
\hline 1 & -2.99649 & -3.59447 & -0.18692 \\
\hline 1 & -0.85379 & -4.42171 & 0.111103 \\
\hline 1 & -0.35961 & -3.42731 & -1.27002 \\
\hline 6 & -0.04314 & -2.49978 & 0.606962 \\
\hline 8 & 1.17963 & -2.45009 & 0.491985 \\
\hline 7 & -0.70751 & -1.75661 & 1.514716 \\
\hline 6 & 0.026239 & -0.82888 & 2.342162 \\
\hline 1 & -1.719 & -1.73282 & 1.520375 \\
\hline 1 & -0.66569 & -0.36092 & 3.046636 \\
\hline 1 & 0.794871 & -1.3474 & 2.921083 \\
\hline 6 & 0.746344 & 0.266477 & 1.557626 \\
\hline 8 & 1.734855 & 0.812541 & 2.038391 \\
\hline 7 & 0.242024 & 0.601156 & 0.343584 \\
\hline & & & \\
\hline
\end{tabular}




\begin{tabular}{|c|c|c|c|}
\hline 6 & 0.971803 & 1.487623 & -0.53469 \\
\hline 1 & -0.49831 & 0.030525 & -0.06387 \\
\hline 1 & 1.990863 & 1.583179 & -0.14522 \\
\hline 6 & 0.382853 & 2.888456 & -0.51989 \\
\hline 8 & -0.69954 & 3.204846 & -0.04749 \\
\hline 6 & 0.996192 & 0.968191 & -1.98821 \\
\hline 1 & -0.0348 & 0.92327 & -2.35965 \\
\hline 1 & 1.531056 & 1.704243 & -2.59691 \\
\hline 6 & 1.624981 & -0.41659 & -2.1724 \\
\hline 1 & 1.049448 & -1.17414 & -1.63047 \\
\hline 1 & 1.539134 & -0.67002 & -3.23325 \\
\hline 6 & 3.102443 & -0.53494 & -1.79391 \\
\hline 1 & 3.526091 & -1.42751 & -2.27026 \\
\hline 1 & 3.655796 & 0.342411 & -2.1498 \\
\hline 7 & 3.265427 & -0.62167 & -0.34004 \\
\hline 1 & 2.555281 & -1.15338 & 0.169202 \\
\hline 6 & 4.392329 & -0.33297 & 0.310344 \\
\hline 7 & 4.372953 & -0.24448 & 1.641628 \\
\hline 1 & 3.495415 & -0.01688 & 2.106088 \\
\hline 1 & 5.231569 & -0.18913 & 2.168671 \\
\hline 7 & 5.525472 & -0.10512 & -0.36565 \\
\hline 1 & 5.647284 & -0.44704 & -1.3073 \\
\hline 1 & 6.340881 & 0.263974 & 0.101188 \\
\hline 8 & 1.170437 & 3.756691 & -1.12235 \\
\hline 1 & 0.77113 & 4.643498 & -1.11554 \\
\hline 1 & -3.51801 & 2.663488 & -0.58041 \\
\hline 1 & -4.35065 & -1.6276 & -2.08731 \\
\hline & & & \\
\hline
\end{tabular}

$b_{2}-y_{3} T S$

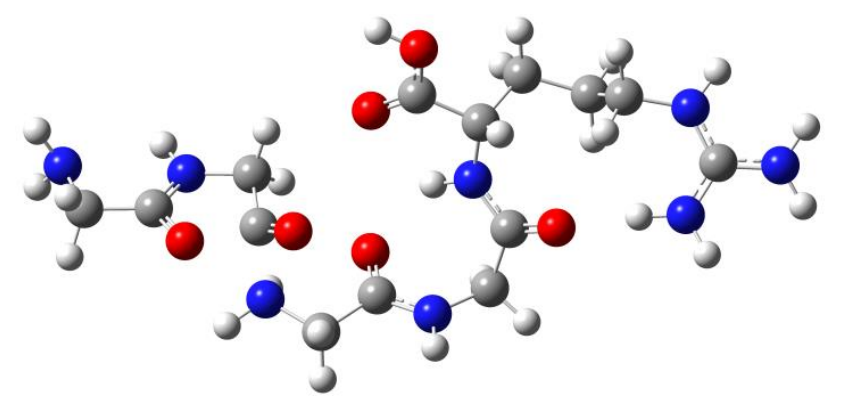

\begin{tabular}{|c|c|c|c|}
\hline $\begin{array}{c}\text { Atom } \\
\text { number }\end{array}$ & $\mathrm{x}$ & $\mathrm{y}$ & $\mathrm{z}$ \\
\hline 7 & 7.528612 & 1.448906 & 0.760675 \\
\hline 6 & 7.098347 & 0.616057 & -0.345459 \\
\hline
\end{tabular}




\begin{tabular}{|c|c|c|c|}
\hline 1 & 8.200860 & 0.984837 & 1.359630 \\
\hline 1 & 7.307871 & 1.050798 & -1.329432 \\
\hline 6 & 5.607571 & 0.414482 & -0.277045 \\
\hline 8 & 4.960783 & -0.259917 & -1.148061 \\
\hline 7 & 4.893101 & 0.931201 & 0.694492 \\
\hline 6 & 3.464131 & 0.754727 & 0.496909 \\
\hline 1 & 5.362024 & 1.516168 & 1.384375 \\
\hline 1 & 2.948481 & 0.372729 & 1.379461 \\
\hline 6 & 3.374573 & -0.237620 & -0.664230 \\
\hline 8 & 2.564373 & -0.312349 & -1.527574 \\
\hline 7 & 3.546607 & -1.937766 & 0.143697 \\
\hline 1 & 3.528042 & -1.866444 & 1.163135 \\
\hline 1 & 4.426060 & -2.356582 & -0.156801 \\
\hline 6 & 2.408368 & -2.739539 & -0.313348 \\
\hline 1 & 2.315072 & -2.615852 & -1.396723 \\
\hline 1 & 2.550241 & -3.799606 & -0.079281 \\
\hline 6 & 1.162275 & -2.214331 & 0.385014 \\
\hline 8 & 1.255876 & -1.396171 & 1.297063 \\
\hline 7 & -0.013384 & -2.721312 & -0.032124 \\
\hline 6 & -1.257300 & -2.432287 & 0.666253 \\
\hline 1 & -0.036996 & -3.342686 & -0.829299 \\
\hline 1 & -1.957255 & -3.248904 & 0.494003 \\
\hline 1 & -1.040612 & -2.357339 & 1.736341 \\
\hline 6 & -1.918585 & -1.142729 & 0.178889 \\
\hline 8 & -2.988255 & -1.167711 & -0.436012 \\
\hline 7 & -1.260564 & -0.008418 & 0.461843 \\
\hline 6 & -1.669757 & 1.274376 & -0.079468 \\
\hline 1 & -0.348835 & -0.063977 & 0.917142 \\
\hline 1 & -2.141934 & 1.104827 & -1.053979 \\
\hline 6 & -0.413305 & 2.098520 & -0.307858 \\
\hline 8 & 0.672833 & 1.820239 & 0.145076 \\
\hline 6 & -2.621112 & 2.055995 & 0.851263 \\
\hline 1 & -2.129110 & 2.163681 & 1.823887 \\
\hline 1 & -2.747887 & 3.063404 & 0.438935 \\
\hline 6 & -3.992529 & 1.406022 & 1.051011 \\
\hline 1 & -3.885252 & 0.374388 & 1.403938 \\
\hline 1 & -4.517342 & 1.957383 & 1.838938 \\
\hline 6 & -4.842353 & 1.438272 & -0.220479 \\
\hline 1 & -4.923682 & 2.461460 & -0.599396 \\
\hline 1 & -4.400228 & 0.832039 & -1.015379 \\
\hline 7 & -6.210125 & 0.961686 & 0.003492 \\
\hline & & & \\
\hline 1 & & & \\
\hline 1 & &
\end{tabular}




\begin{tabular}{|l|l|l|l|}
\hline 1 & -6.863785 & 1.609534 & 0.423208 \\
\hline 6 & -6.654438 & -0.272250 & -0.238673 \\
\hline 7 & -7.969368 & -0.521358 & -0.150147 \\
\hline 1 & -8.323520 & -1.465854 & -0.157447 \\
\hline 1 & -8.645978 & 0.226334 & -0.117617 \\
\hline 7 & -5.819634 & -1.251919 & -0.568290 \\
\hline 1 & -6.184887 & -2.122513 & -0.924750 \\
\hline 1 & -4.803883 & -1.165276 & -0.496623 \\
\hline 8 & -0.671908 & 3.181739 & -1.034973 \\
\hline 1 & 0.137964 & 3.706734 & -1.140900 \\
\hline 1 & 7.935985 & 2.323512 & 0.451052 \\
\hline 1 & 2.985152 & 1.682072 & 0.173989 \\
\hline 1 & 7.561794 & -0.376817 & -0.346686 \\
\hline
\end{tabular}

G5R

Minimum

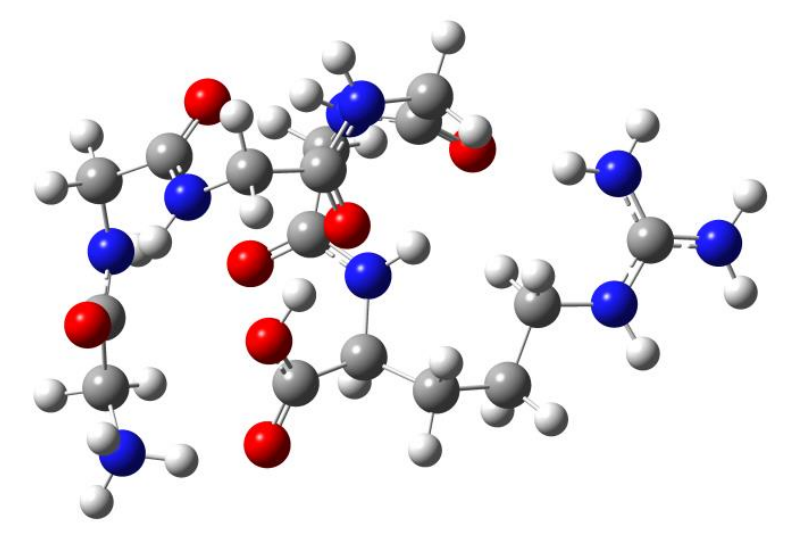

\begin{tabular}{|c|c|c|c|}
\hline $\begin{array}{c}\text { Atom } \\
\text { number }\end{array}$ & $\mathrm{x}$ & $\mathrm{y}$ & $\mathrm{z}$ \\
\hline 7 & 4.009277 & -3.25501 & -0.49085 \\
\hline 1 & 4.425617 & -4.18237 & -0.39381 \\
\hline 1 & 3.022775 & -3.34898 & -0.85542 \\
\hline 1 & 4.575893 & -2.68122 & -1.13656 \\
\hline 6 & 3.924779 & -2.52005 & 0.800296 \\
\hline 1 & 2.955173 & -2.71589 & 1.264435 \\
\hline 1 & 4.72599 & -2.85076 & 1.465277 \\
\hline 6 & 4.136654 & -1.04371 & 0.48007 \\
\hline 8 & 4.74039 & -0.73009 & -0.54405 \\
\hline 7 & 3.704497 & -0.17898 & 1.404645 \\
\hline 6 & 4.002435 & 1.23885 & 1.28678 \\
\hline 1 & 2.99043 & -0.47462 & 2.065381 \\
\hline
\end{tabular}




\begin{tabular}{|c|c|c|c|}
\hline 1 & 3.937084 & 1.698672 & 2.272298 \\
\hline 1 & 5.019285 & 1.347578 & 0.899914 \\
\hline 6 & 3.010964 & 1.960104 & 0.380196 \\
\hline 8 & 2.176404 & 2.74068 & 0.83741 \\
\hline 7 & 3.08202 & 1.697976 & -0.94239 \\
\hline 6 & 2.248876 & 2.454633 & -1.85578 \\
\hline 1 & 3.776335 & 1.040177 & -1.28467 \\
\hline 1 & 2.501754 & 3.51908 & -1.80629 \\
\hline 1 & 2.429964 & 2.097611 & -2.87185 \\
\hline 6 & 0.773177 & 2.240488 & -1.53976 \\
\hline 8 & 0.278837 & 1.105949 & -1.47947 \\
\hline 7 & 0.019306 & 3.326447 & -1.30556 \\
\hline 6 & -1.37411 & 3.190863 & -0.91787 \\
\hline 1 & 0.459545 & 4.236157 & -1.27706 \\
\hline 1 & -1.76762 & 4.179058 & -0.66891 \\
\hline 1 & -1.96716 & 2.780506 & -1.73906 \\
\hline 6 & -1.57131 & 2.242145 & 0.266606 \\
\hline 8 & -2.61792 & 1.580611 & 0.353721 \\
\hline 7 & -0.56704 & 2.13828 & 1.140134 \\
\hline 6 & -0.48542 & 1.084846 & 2.138962 \\
\hline 1 & 0.300985 & 2.642051 & 0.963249 \\
\hline 1 & 0.246737 & 1.374624 & 2.892126 \\
\hline 1 & -1.46135 & 0.957161 & 2.616734 \\
\hline 6 & -0.04159 & -0.25986 & 1.544074 \\
\hline 8 & 0.917648 & -0.89426 & 1.975016 \\
\hline 7 & -0.80087 & -0.7219 & 0.521325 \\
\hline 6 & -0.56899 & -2.01772 & -0.07853 \\
\hline 1 & -1.49655 & -0.10118 & 0.125809 \\
\hline 1 & -0.66128 & -2.79766 & 0.686705 \\
\hline 6 & 0.856206 & -2.17489 & -0.60792 \\
\hline 8 & 1.361874 & -3.28888 & -0.62902 \\
\hline 6 & -1.55465 & -2.2971 & -1.22556 \\
\hline 1 & -1.48054 & -1.48871 & -1.96608 \\
\hline 1 & -1.21801 & -3.21342 & -1.71877 \\
\hline 6 & -3.01965 & -2.49604 & -0.80878 \\
\hline 1 & -3.55579 & -2.85507 & -1.69556 \\
\hline 1 & -3.09307 & -3.27838 & -0.04505 \\
\hline 6 & -3.69078 & -1.2166 & -0.3167 \\
\hline 1 & -3.33368 & -0.94342 & 0.681128 \\
\hline 1 & -3.45848 & -0.38994 & -1.00215 \\
\hline 7 & -5.14307 & -1.37559 & -0.23679 \\
\hline & & & \\
\hline 1 & & & \\
\hline & & &
\end{tabular}




\begin{tabular}{|c|c|c|c|}
\hline 1 & -5.5687 & -2.19522 & -0.64946 \\
\hline 6 & -5.94008 & -0.42025 & 0.246549 \\
\hline 7 & -5.42646 & 0.751571 & 0.611875 \\
\hline 1 & -6.00631 & 1.433802 & 1.077988 \\
\hline 1 & -4.45438 & 1.011483 & 0.440344 \\
\hline 7 & -7.25343 & -0.64683 & 0.371564 \\
\hline 1 & -7.89273 & 0.09301 & 0.620938 \\
\hline 1 & -7.64466 & -1.56743 & 0.238503 \\
\hline 8 & 1.525985 & -1.1388 & -1.05423 \\
\hline 1 & 1.018222 & -0.27447 & -1.1487 \\
\hline
\end{tabular}

$a_{1}-y_{5}$ TS

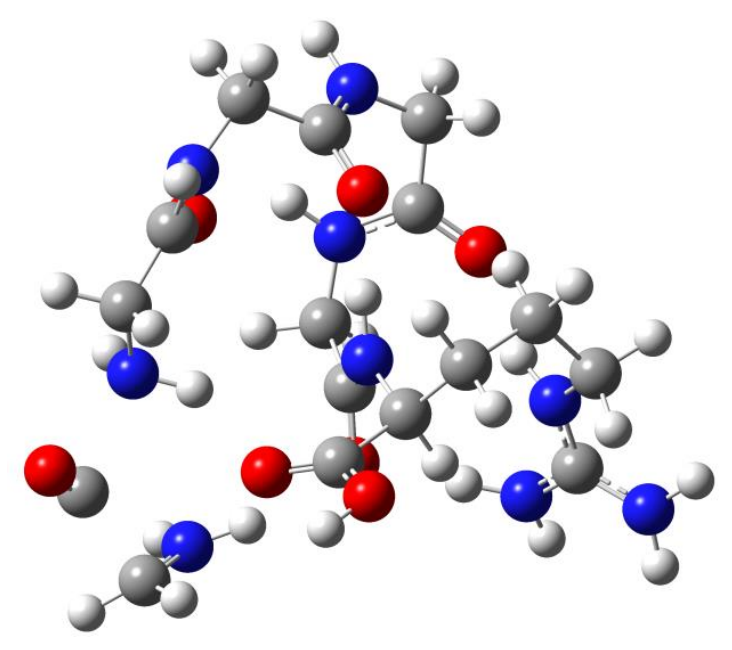

\begin{tabular}{|c|c|c|c|}
\hline $\begin{array}{c}\text { Atom } \\
\text { number }\end{array}$ & $\mathrm{x}$ & $\mathrm{y}$ & $\mathrm{z}$ \\
\hline 7 & -1.725988 & -3.338914 & -1.754661 \\
\hline 1 & -1.873915 & -3.201066 & -2.745787 \\
\hline 1 & -0.890509 & -2.918469 & -1.345109 \\
\hline 6 & -2.597627 & -3.998089 & -1.033047 \\
\hline 1 & -3.352769 & -4.583693 & -1.546835 \\
\hline 6 & -4.015106 & -2.763153 & -0.156163 \\
\hline 8 & -4.817124 & -2.535067 & 0.613641 \\
\hline 7 & -3.174513 & -0.749268 & -0.820983 \\
\hline 1 & -3.288997 & -0.406543 & -1.772334 \\
\hline 1 & -2.181824 & -0.900762 & -0.650251 \\
\hline 6 & -3.676228 & 0.239787 & 0.121259 \\
\hline 1 & -4.753098 & 0.377676 & -0.028254 \\
\hline 6 & -2.974346 & 1.584709 & -0.055459 \\
\hline 8 & -2.374279 & 1.841320 & -1.099125 \\
\hline
\end{tabular}




\begin{tabular}{|l|c|c|c|}
\hline 7 & -3.025580 & 2.467920 & 0.960891 \\
\hline 6 & -2.269292 & 3.701083 & 0.862346 \\
\hline 1 & -3.448337 & 2.211816 & 1.841428 \\
\hline 1 & -2.674441 & 4.332760 & 0.065880 \\
\hline 6 & -0.798293 & 3.370165 & 0.601117 \\
\hline 8 & -0.250102 & 2.425376 & 1.168189 \\
\hline 7 & -0.142663 & 4.124643 & -0.304566 \\
\hline 6 & 1.236001 & 3.814405 & -0.640378 \\
\hline 1 & -0.615562 & 4.887721 & -0.769126 \\
\hline 1 & 1.602847 & 4.546329 & -1.363182 \\
\hline 6 & 1.402824 & 2.411163 & -1.224320 \\
\hline 8 & 2.482405 & 1.824395 & -1.143799 \\
\hline 7 & 0.319998 & 1.853769 & -1.796196 \\
\hline 6 & 0.348732 & 0.487686 & -2.248400 \\
\hline 1 & -0.585774 & 2.307058 & -1.733416 \\
\hline 1 & -0.589267 & 0.276406 & -2.769020 \\
\hline 6 & 0.527516 & -0.555972 & -1.147909 \\
\hline 8 & 0.804054 & -1.725663 & -1.466480 \\
\hline 7 & 0.395500 & -0.173661 & 0.127888 \\
\hline 6 & 0.787489 & -1.038512 & 1.223834 \\
\hline 1 & 0.161587 & 0.796773 & 0.352837 \\
\hline 1 & 1.547451 & -1.732258 & 0.837806 \\
\hline 6 & -0.362089 & -1.952470 & 1.630817 \\
\hline 8 & -1.328354 & -2.191306 & 0.931375 \\
\hline 6 & 1.365343 & -0.228014 & 2.394446 \\
\hline 1 & 0.577095 & 0.411878 & 2.807060 \\
\hline 1 & 1.649335 & -0.933576 & 3.180190 \\
\hline 6 & 2.562458 & 0.657880 & 2.030309 \\
\hline 1 & 2.265207 & 1.440496 & 1.324330 \\
\hline 1 & 2.873214 & 1.175230 & 2.942616 \\
\hline 6 & 3.789940 & -0.073516 & 1.483098 \\
\hline 1 & 4.662745 & 0.587284 & 1.544106 \\
\hline 1 & 3.992964 & -0.971910 & 2.078392 \\
\hline 7 & 3.575946 & -0.485497 & 0.090346 \\
\hline 1 & 3.084282 & 0.187377 & -0.508899 \\
\hline 6 & 4.225083 & -1.479930 & -0.509639 \\
\hline 7 & 3.775275 & -1.937398 & -1.683993 \\
\hline 1 & 4.347758 & -2.538589 & -2.257800 \\
\hline 1 & 2.788396 & -1.838975 & -1.912386 \\
\hline 7 & 5.293318 & -2.043377 & 0.065876 \\
\hline 1 & 5.820194 & -1.552610 & 0.773714 \\
\hline & & & \\
\hline 1 & & & \\
\hline 1 & & & \\
\hline & & &
\end{tabular}




\begin{tabular}{|c|c|c|c|}
\hline 1 & 5.684848 & -2.902637 & -0.291516 \\
\hline 8 & -0.158781 & -2.515491 & 2.813014 \\
\hline 1 & -0.884978 & -3.124911 & 3.025590 \\
\hline 1 & -3.534463 & -0.132879 & 1.141397 \\
\hline 1 & -2.291949 & -4.304936 & -0.038970 \\
\hline 1 & -2.348146 & 4.238380 & 1.809919 \\
\hline 1 & 1.872891 & 3.859781 & 0.247001 \\
\hline 1 & 1.167171 & 0.327481 & -2.957586 \\
\hline
\end{tabular}

$\mathrm{b}_{2}-\mathrm{y}_{4} \mathrm{TS}$

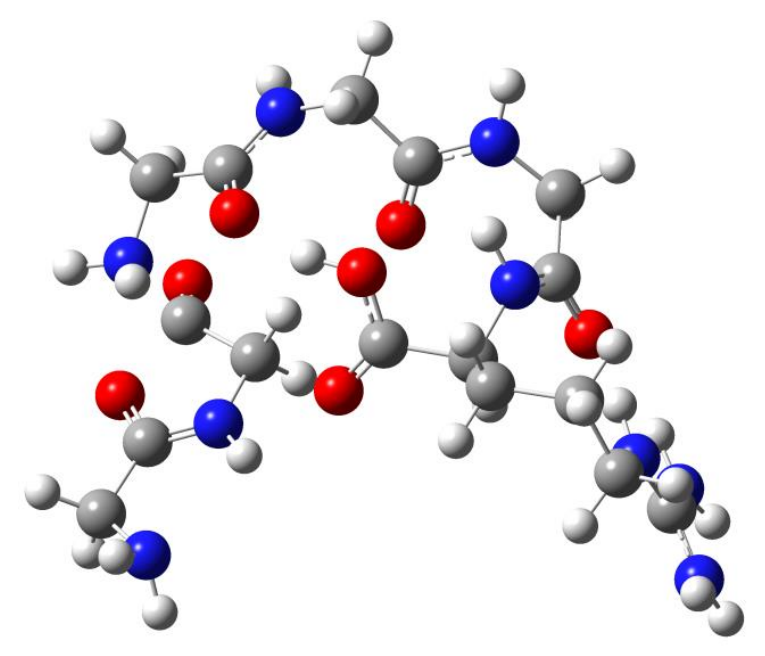

\begin{tabular}{|c|c|c|c|}
\hline $\begin{array}{c}\text { Atom } \\
\text { number }\end{array}$ & $\mathrm{x}$ & $\mathrm{y}$ & $\mathrm{z}$ \\
\hline 7 & 2.691758 & 4.572008 & 0.987197 \\
\hline 1 & 2.335320 & 5.436385 & 0.597970 \\
\hline 1 & 2.855137 & 4.717495 & 1.975871 \\
\hline 6 & 3.876144 & 4.103453 & 0.294632 \\
\hline 1 & 4.190545 & 4.760477 & -0.524415 \\
\hline 6 & 3.617278 & 2.755602 & -0.322066 \\
\hline 8 & 4.518587 & 2.122921 & -0.972649 \\
\hline 7 & 2.457761 & 2.153018 & -0.217415 \\
\hline 6 & 2.396226 & 0.931674 & -0.995437 \\
\hline 1 & 1.689499 & 2.556694 & 0.315370 \\
\hline 1 & 1.914502 & 0.119253 & -0.450408 \\
\hline 6 & 3.855275 & 0.632276 & -1.327496 \\
\hline 8 & 4.305035 & 0.050795 & -2.260245 \\
\hline 7 & 4.574154 & -0.078310 & 0.209368 \\
\hline 1 & 4.117464 & 0.219131 & 1.077465 \\
\hline
\end{tabular}




\begin{tabular}{|l|l|l|l|}
\hline 1 & 5.534405 & 0.266591 & 0.191051 \\
\hline 6 & 4.531936 & -1.541370 & 0.116057 \\
\hline 1 & 4.729797 & -1.818823 & -0.924137 \\
\hline 6 & 3.157509 & -1.991947 & 0.591364 \\
\hline 8 & 2.562378 & -1.317824 & 1.435466 \\
\hline 7 & 2.692609 & -3.138887 & 0.077512 \\
\hline 6 & 1.385431 & -3.652680 & 0.411369 \\
\hline 1 & 3.201391 & -3.570820 & -0.682632 \\
\hline 1 & 1.367513 & -4.728851 & 0.224349 \\
\hline 1 & 1.201251 & -3.490797 & 1.479330 \\
\hline 6 & 0.287176 & -2.928451 & -0.376114 \\
\hline 8 & 0.461723 & -1.826821 & -0.870902 \\
\hline 7 & -0.917386 & -3.565531 & -0.422740 \\
\hline 6 & -1.997645 & -3.005655 & -1.218114 \\
\hline 1 & -0.986344 & -4.527247 & -0.118559 \\
\hline 1 & -1.687898 & -2.849631 & -2.255884 \\
\hline 1 & -2.841577 & -3.699487 & -1.211082 \\
\hline 6 & -2.462716 & -1.643210 & -0.718814 \\
\hline 8 & -3.025023 & -0.862198 & -1.488968 \\
\hline 7 & -2.248190 & -1.360638 & 0.584083 \\
\hline 6 & -2.024492 & 0.007791 & 1.048322 \\
\hline 1 & -1.758720 & -2.070586 & 1.118065 \\
\hline 1 & -2.232601 & 0.685259 & 0.217281 \\
\hline 6 & -0.529598 & 0.142742 & 1.340327 \\
\hline 8 & 0.138295 & 1.095710 & 1.008115 \\
\hline 6 & -2.863663 & 0.363604 & 2.285479 \\
\hline 1 & -2.546205 & -0.283554 & 3.110930 \\
\hline 1 & -2.618847 & 1.391587 & 2.577819 \\
\hline 6 & -4.380036 & 0.210552 & 2.113448 \\
\hline 1 & -4.622143 & -0.803621 & 1.772919 \\
\hline 1 & -4.835632 & 0.316008 & 3.101841 \\
\hline 6 & -5.070815 & 1.224335 & 1.198882 \\
\hline 1 & -6.151520 & 1.173538 & 1.378425 \\
\hline 1 & -4.726706 & 2.241234 & 1.430351 \\
\hline 7 & -4.805221 & 0.925763 & -0.208340 \\
\hline 1 & -4.200889 & 0.141540 & -0.458866 \\
\hline 6 & -5.295139 & 1.594329 & -1.245308 \\
\hline 7 & -4.912332 & 1.245495 & -2.478041 \\
\hline 1 & -5.344787 & 1.643022 & -3.297460 \\
\hline 1 & -4.249846 & 0.486181 & -2.597276 \\
\hline 7 & -6.141637 & 2.613737 & -1.064307 \\
\hline & & & \\
\hline 1 & & & \\
\hline 1 & &
\end{tabular}




\begin{tabular}{|c|c|c|c|}
\hline 1 & -6.499879 & 2.840052 & -0.149289 \\
\hline 1 & -6.506401 & 3.137141 & -1.845970 \\
\hline 8 & -0.061273 & -0.916844 & 1.992978 \\
\hline 1 & 0.924316 & -0.929493 & 1.982546 \\
\hline 1 & 5.289233 & -2.001575 & 0.759746 \\
\hline 1 & 4.749622 & 3.998581 & 0.947690 \\
\hline 1 & 1.874389 & 1.093565 & -1.943798 \\
\hline
\end{tabular}

